\title{
The Formation of Curved Polymer Crystals: Polyoxymethylene
}

\author{
F. Khoury and J. D. Barnes
}

\author{
Institute for Materials Research, National Bureau of Standards, Washington, D.C. 20234
}

(November 14, 1973)

\begin{abstract}
An optical and electron microscopical study is presented of the habits exhibited by chain-folded polyoxymethylene crystals grown from hot 0.02 percent solutions of the polymer in orthodichlorobenzene when these solutions are cooled to temperatures $\left(T_{c}\right)$ between 140 and $80^{\circ} \mathrm{C}$ inclusive. In contrast with the lamellar crystals formed in the $T_{c}=140$ and $120^{\circ} \mathrm{C}$ preparations, which crystals were six-sectored and nearly planar, the crystals formed below $T_{c}=120{ }^{\circ} \mathrm{C}$ exhibited multisectored dendritic habits and were all the more pronouncedly curved the lower the temperature at which they grew. The most pronouncedly curved crystals which were observed were hollow bowl shaped dendrites having a radius of curvature of $\sim 2 \mu \mathrm{m}$ which were formed in the $80^{\circ} \mathrm{C}$ preparations. The possible origins of why the lamellar crystals of polyoxymethylene were all the more pronouncedly curved the lower the crystallization temperature are considered in the light of conjectures which have been previously advanced concerning the formation of curved crystals of poly(4-methylpentene- 1$)$. Among the features which are discussed is the role played by the bulkiness of the chain folds.
\end{abstract}

Key words: Chain-folded; curved; crystal; electron microscopy; optical microscopy; polymer; polyoxymethylene; solution grown.

\section{Introduction}

We report in this paper the outcome of a study of the habits exhibited by lamellar crystals of polyoxymethylene (POM) grown at different supercoolings from hot 0.02 percent solutions of the polymer in orthodichlorobenzene [1]. ${ }^{1}$ This survey of the effect of supercooling on the growth habits of POM crystals was undertaken with the specific purpose of determining whether increasing the degree of supercooling at which POM is crystallized from solutions having the same concentration of polymer leads to the formation of lamellar crystals which are all the more pronouncedly curved, i.e., have a smaller radius of curvature, the higher the conditions of supercooling (lower crystallization temperatures) which prevail during the growth of the crystals. We first observed the incidence of this latter phenomenon in a previous study of the crystallization of poly(4-methylpentene-1) (P4MP) from solution [2]. Briefly, it was found that the habits of P4MP crystals ranged from very nearly planar chain-folded lamellae which were formed at high crystallization temperatures, to distinctly curved hollow bowl-shaped multilayered lamellar crystals having a radius of curvature of $\sim 2.5 \mu \mathrm{m}$ which were formed at much lower temperatures. Our observations on this effect of increasing supercooling on the habits of P4MP crystals have been described and discussed in detail in a previous paper

${ }^{1}$ Figures in brackets indicate the literature references at the end of this paper.
[2]. The objective of the present study was to determine whether an analogous phenomenon manifests itself when POM is crystallized from solution at different supercoolings. This has indeed been found to be the case as will be described in Section 3 of this paper.

We shall not dwell at this stage on the nature of the considerations which originally led us to speculate that there might exist a phenomenological similarity between the effect of supercooling on the character of solution grown crystals of P4MP and POM insofar as the formation of curved lamellar crystals is concerned. This matter is deferred to Section 4 where the observations derived from the present study on the formation and characteristics of curved crystals of POM are discussed in the light of conjectures which we have previously advanced [2] concerning the origins of curvature in P4MP crystals and the causes of the enhancement of this feature the greater the supercooling at which the crystals of that polymer are grown from solution. We shall limit ourselves at this stage to merely pointing out previously reported observations concerning the habits of solution-grown crystals of POM, which observations anteceded for the most part the undertaking of the present study.

POM has been crystallized from solution in the form of hexagonal-shaped chain-folded lamellar crystals which are six-sectored ${ }^{2}[3-8]$. As indicated schemat- 


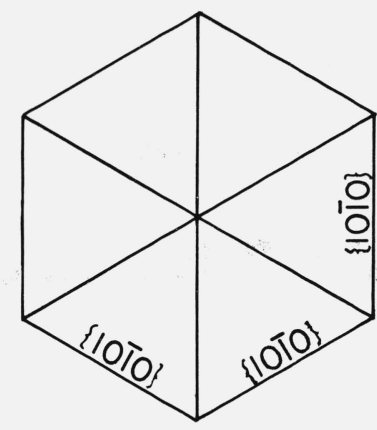

(a)

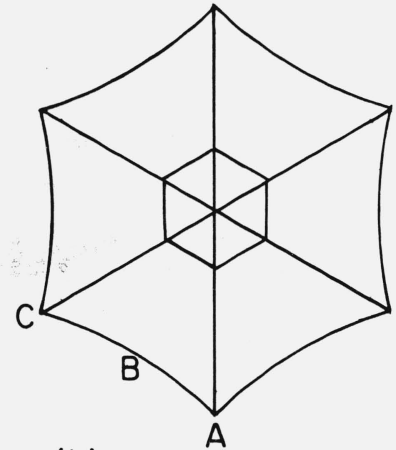

(b)

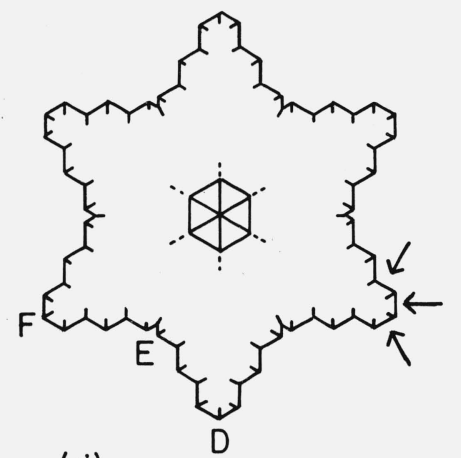

(d)

(c)

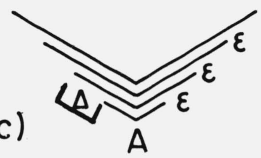

FIGURE 1. Schematic representation of lateral growth habits exhibited by chain-folded crystals of POM.

\begin{abstract}
(a) Hexagonal shaped six-sectored lamella bound by $\{10 \overline{1} 0\}$ growth faces. (b) Star-shaped six-sectored habit, SS, resulting from the preferential initiation of new. $\{10 \overline{1} 0\}$ planes at the leading corners of a hexagonal shaped six-sectored precursor crystal. (c) Larger scale representation of a leading comer of an SS type lamella (e.g., corner A in (b)) depicting the lag $\Delta$ between the extremities $\epsilon$ of successive $\{10 \overline{1} 0\}$ planes originating from the corner. (d) Starlike dendritic multisectored habit, SD, resulting from the generation of new sectored outgrowths at the leading corners of a hexagonal shaped six-sectored precursor crystal; the arrows point to $\{10 \overline{1} 0\}$ microfacets along which the chain molecules fold.
\end{abstract}

ically in figure la, these crystals are bound laterally by $\{10 \overline{1} 0\}$ faces at which growth occurs through the accretion of chain molecules which fold along correspondingly parallel $\{10 \overline{1} 0\}$ planes. Several observations, namely, the diffraction contrast characteristics manifested in both dark-field and bright-field electron micrographs of six-sectored crystals sedimented and dried on a flat substrate $[5,6]$, the appearance exhibited by the crystals when they are examined as they sediment and dry on a substrate during the evaporation of the liquid in which they were grown [6], the not infrequent incidence of pleats in sedimented crystals [6-8], ${ }^{3}$ the slightly 'warped' appearance of, and the shadow density distribution about, some sedimented crystals [4], have been adduced as evidence that six-sectored chain-folded lamellae of POM are not perfectly flat as grown [4-7]. The matter of the as-grown conformation (nonplanar character) of sixsectored chain-folded lamellae of POM will be considered further in Section 3.2 of this paper in the light of both the aforementioned observations as well as some similar and additional ones obtained in the present study which includes an examination of such crystals as viewed under the optical microscope while they are in suspension in liquid. It is appropriate, however, by way of background, to summarize at this juncture the possibilities and conjectures which have been previously advanced concerning the nature and origins of the nonplanar character of six-sectored lamellar crystals of POM.

The possibility was raised in an earlier paper [4]

${ }^{3}$ Some of the monolayered crystals in [6] exhibit pleats, others do not. All the monolayered crystals in figure $1[7]$ exhibit pleats. None of the monolayered crystals in figure $3 \mathrm{a}$ [8] exhibit pleats. that six-sectored crystals of POM may be hollow pyramidal in character, the implication being that the chain folds in successive $\{1010\}$ fold planes in the constituent fold-domains (sectors) in these crystals might be regularly staggered. This latter possibility remained conjectural (e.g., see review [7]) for lack of definitive evidence. More recently another source of the nonplanar character of six-sectored lamellae has come to light. Briefly, an analysis due to Bassett [5] of $\{10 \overline{1} 0\}$ dark field Moiré patterns exhibited by bilayered crystals, whose two constituent superimposed chain-folded layers were six-sectored and slightly rotated relative to one another about an axis perpendicular to the layers, indicated that there exists in each constituent sector of the six-sectored layers a fractional difference of $\sim 3.2 \times 10^{-3}$ between the interplanar spacing of the $\{10 \overline{1} 0\}$ planes which are fold planes and the interplanar spacing of the other two families of $\{10 \overline{1} 0\}$ planes in each sector which are not fold planes. ${ }^{4}$ This distortion of the metrically hexagonal unit cell [9] (or more accurately the subcell) of POM in the constituent sectors of six-sectored lamellae has been attributed by Bassett et al. $[5,6]$ to an asymmetric bulkiness of the chain folds. Bassett et al. $[5,6]$ have suggested that the nonplanar character of six-sectored lamellae is a consequence (in part at any rate) of slight buckling caused by internal stresses associated with the distortion of the subcell in its constitutent fold domains. In the absence of a regular staggering of chain-folds such buckling may be envisaged to impart a slight conicalness to the lamella

${ }^{4}$ For the sake of brevity we shall denote the $\{10 \overline{1} 0\}$ planes along which the chain mole cules are folded in POM crystals as $\{10 \overline{1} 0\}_{f}$ planes in the rest of this paper. The $\{10 \overline{1} 0\}$ planes which are not fold planes will be denoted $\{10 \overline{1} 0\}_{\text {nf }}$ 
which would have otherwise been flat. The possibility was however advanced by Bassett et al. [6], that the nonplanar character of six-sectored lamellae might be due to a combination of two factors, namely, a regular staggering of chain folds coupled with an additional slight buckling of the resulting hexagonally based hollow pyramidal crystal, which buckling is associated with the distortion of the subcell in its constituent sectors as mentioned above. We shall not pursue the matter of the nature and origins of the as-grown conformation of six-sectored lamellae any further at this stage. As pointed out earlier this aspect will be considered and discussed again in Section 3.2 where observations bearing on the question of whether or not regular fold staggering occurs in six-sectored crystals of POM will be presented.

Having outlined some of the morphological characteristics of six-sectored lamellar crystals of POM and having given an indication of some of the views and conjectures pertaining to the nature and origins of their nonplanar character, we pass on to a summary of previous observations on the nature of POM crystals whose habits differ from but are related to that of the hexagonally shaped six-sectored crystals discussed in the previous two paragraphs. Such crystals, which have been described by Reneker and Geil [4], consisted of a basal (leading) chain-folded lamella upon which additional chain-folded terraces of much smaller lateral extent than that of the basal lamella had developed in spiral-like fashion through the agency of screw dislocations generated intermittently at the periphery of the basal lamella during its outward growth. The basal lamella in these crystals exhibited a distinct six-pointed star-like overall shape; its sides were not smooth but appeared faintly microfaceted. In addition, the terraces which developed upon the basal lamella exhibited incipient star-like lateral profiles. Reneker and Geil [4] have pointed out that such crystals evolve from a hexagonal six-sectored precursor lamella bound laterally by $\{10 \overline{1} 0\}$ faces grow. ing under conditions (high supercooling, see later) which favor the preferential deposition of chain molecules, and hence the preferential initiation of new $\{10 \overline{1} 0\}_{f}$ planes, at the corners of that lamella. Given this latter feature, the precursor may be envisaged to develop into one of two distinguishable types of sixpointed star-shaped lamellae (which are depicted schematically in figure $1 \mathrm{~b}$ and figure $1 \mathrm{~d}$ ) depending on the relative magnitudes of the rate of initiation $R_{i}$ of new $\{10 \overline{1} 0\}_{f}$ planes at the leading corners of the precursor and the rate $R_{p}$ at which these planes propagate therefrom along its $\{10 \overline{1} 0\}$ faces.

A schematic representation of the star-shaped sixsectored type of lamella (see illustration in figure 11, ref. [4]) which would be expected to develop when the ratio $R=R_{i} / R_{p}$ is relatively small is depicted in figure lb. For the sake of brevity and in order to avoid confusion such star-shaped six-sectored lamellae will be referred to as type (SS) lamellae in this paper. The formation of this type of lamella would be expected to occur over a range of values of the ratio $R$. Within this range the larger the ratio $R$, the smaller will be the lateral dimensions which the precursor will attain before a change in its lateral shape from hexagonal to star-like (SS) will manifest itself. Furthermore as may be visualized with reference to figure $l b$ and figure lc the lag in distance $(\Delta)$ between the advancing extremities $(\epsilon)$ of successive $\{10 \overline{1} 0\}_{f}$ planes originating from the corners (e.g., A) will tend to be smaller and, correspondingly, the fronts (e.g., $\mathrm{AB}$ and $\mathrm{CB}$ ) which delineate the extremities of the succession of parallel $\{10 \overline{1} 0\}_{f}$ planes advancing in staggered formation from each corner will tend to be steeper, i.e., more pronouncedly reentrant, in the fully grown lamella. As pointed out by Geil [7] however, this latter trend will ultimately break down in that at larger values of $R$ and hence smaller $(\Delta)$ reentrant $\{10 \overline{1} 0\}$ microfacets along which chain folding occurs will develop, as a consequence of which a star-shaped dendritic lamella will evolve from the hexagonal precursor. A schematic representation of such a lamella is depicted in figure 1d. Lamellae of this type will henceforth be referred to as (SD) lamellae in order to distinguish them from the (SS) lamellae discussed above from which they differ in the following two interrelated respects. First, in contrast with the sides (e.g., ABC, figure lb) of (SS) lamellae, the sides (e.g. DEF, figure 1d) of (SD) lamellae are microfaceted, the microfacets along which the chain molecules fold are $\{10 \overline{1} 0\}$ microfacets. Second, in contrast with the (SS) lamellae which are six-sectored, the (SD) lamellae are multisectored. This latter feature is an inherent consequence of their lateral mode of propagation which is characterized by the repeated generation of new sectored outgrowths bound by $\{10 \overline{1} 0\}$ facets at the leading corners of the developing lamella throughout its growth. ${ }^{5}$ A progressive transformation in the character of the developing lamella from hexagonal six-sectored, to (SS), and then to (SD) may be envisaged to occur during its early stages of development, the onset of the (SD) character manifesting itself at a smaller stage in the lateral growth of the lamella the higher the rate of initiation of $\{10 \overline{1} 0\}_{f}$ planes at its leading corners relative to the rate at which the planes propagate along the sides of the crystal.

Judging from the microfaceted character of the periphery of the basal lamella as well as some of the terraces which developed therefrom in the starshaped type of crystal shown by Reneker and Geil (fig. 6, ref. [4]), that crystal may be classified as an incipient (SD) crystal. Although the range of conditions which lead to the formation of this type of crystal rather than hexagonal six-sectored ones were not investigated at the time, the dendritic character of these (SD) crystals indicated in itself that their formation occurs at high supercoolings. Two additional features exhibited in these crystals should be mentioned. First, denoting the bisector of the line joining adjacent leading corners of the basal lamella as a "slow growth line," Reneker and Geil [4] have pointed

5 The (SS) and (SD) types of lamellae depicted in fig. lb and fig. 1d have been previously referred to as "star-shaped" crystals and "dendritic" crystals respectively $[4,7]$. 
out that the spiral terraces which developed upon the basal lamella originated from screw dislocations which were usually situated along the slow growth lines, and that in most instances these dislocations occurred in pairs of opposite hand lying along the slow growth lines. Second, and of particular interest in connection with the present study, the incidence of pleats in the interior of such crystals which had been deposited and dried upon a substrate was noted [4]. The occurrence of these pleats provided us at the outset of the present study with an indication that type (SD) crystals were probably not planar as-grown. The as-grown conformation of such crystals remained undetermined however. As will be seen in what ensues the present study sheds further light upon the matter of the asgrown conformations of multisectored dendritic lamellar crystals of POM as well as upon how the conformations of such crystals differ from hexagonal six-sectored and (SS) type crystals.

\section{Experimental Details}

\subsection{Materials and Preparation Methods}

The polyoxymethylene used in this study was Delrin (Type 500-NC-10) ${ }^{6}$ supplied in the form of pellets $\sim 2 \mathrm{~mm}$ in length and $\sim 2 \mathrm{~mm}$ in diameter. In order to facilitate rapid dissolution of the polymer in solvent, the pellets were cut into slices $\sim 40 \mu \mathrm{m}$ in thickness. Distilled orthodichlorobenzene, in which it was initially established the polymer dissolves at $180^{\circ} \mathrm{C}$ but not at room temperature, was used as a solvent. Solutions having a polymer concentration of 0.02 percent (expressed as grams of polymer per $100 \mathrm{ml}$ of solvent at room temperature) were prepared in $10 \mathrm{ml}$ quantities by mixing appropriate amounts of the solvent and sliced polymer at room temperature and then immersing the mixture (contained in a covered test tube, internal diameter $\sim 2 \mathrm{~cm}$ ) for half an hour in a bath held at $180^{\circ} \mathrm{C}$.

The objective of the present study was to establish from the outset a procedure under which the polymer crystallizes from the above mentioned solutions at some predetermined temperature in the form of hexagonal shaped six-sectored lamellar crystals (i.e., such as that depicted schematically in fig: la), and then to proceed therefrom with a survey of the habits exhibited by crystals grown at higher supercoolings. Accordingly, exploratory experiments were carried out which resulted in the adoption of a procedure which yielded, upon crystallization at $140^{\circ} \mathrm{C}$, hexagonal six-sectored lamellar crystals which were of suitable size for examination by both optical and electron microscopical techniques. This procedure was as follows. After being held at $180^{\circ} \mathrm{C}$ for half an hour a 0.02 percent solution of the POM in orthodichlorobenzene was transferred to a bath held at $120^{\circ} \mathrm{C}$ and kept in that bath for 1 hour; the solution exhibited a

${ }^{6}$ Trade names identified in this paper are given solely for indicating the source and type of materials or instruments used. This in no way implies endorsement or recommendation by the National Bureau of Standards. distinctly cloudy appearance indicative of the occurrence of crystallization within the first five minutes. The resulting preparation was then transferred to and kept for 15 minutes in another bath held at 153 ${ }^{\circ} \mathrm{C}$ at which temperature (the 'seeding' temperature, henceforth denoted $T_{s}$ ) the polymer dissolved. In order to ensure uniform heating throughout the preparation after it was transferred from the bath at $120{ }^{\circ} \mathrm{C}$ to the bath at $T_{s}=153{ }^{\circ} \mathrm{C}$ it was shaken continuously for about 2 minutes; within that period the polymer dissolved and the solution became clear. After 15 minutes at $153^{\circ} \mathrm{C}$ had elapsed the resulting solution was transferred to the final crystallization bath held at $140{ }^{\circ} \mathrm{C}$ at which temperature the occurrence of crystallization manifested itself (as evidenced by the distinct cloudiness of the preparation) within 15 minutes.

The same preparative procedure as that described above was used in crystallizing the polymer at higher supercoolings. In these experiments the preparations were transferred from the bath at $T_{s}=153{ }^{\circ} \mathrm{C}$ to a bath held at one of the following temperatures: 120 , 114,100 , and $80{ }^{\circ} \mathrm{C}$. These preparations as well as the $140{ }^{\circ} \mathrm{C}$ preparations were kept in the final crystallization bath for several hours. For the sake of brevity in what ensues the temperature of the final crystallization bath will henceforth be denoted $T_{c}$.

Table 1 summarizes the crystallization conditions adopted in this study. This crystallization procedure is akin in principle to the self-seeding procedure used by Blundell et al. in the case of polyethylene [10]. All the bath temperatures were controlled to within \pm 0.1 ${ }^{\circ} \mathrm{C}$. For reasons which will not be dwelt on at this stage (they will be mentioned in Section 3) two series of experiments were carried out which differed only in the treatment of the preparations after they had been maintained at $T_{c}$ for several hours. As indicated in table 1 , in one series the preparations were removed after several hours (usually 24 hours) from the $T_{c}$ bath and allowed to cool to room temperature under ambient conditions, whereas in the other series the mother liquor was exchanged isothermally at $T_{c}$ with fresh solvent (preheated to $T_{c}$, see below) before the preparation was allowed to cool to room temperature under ambient conditions, the purpose of this exchange being to eliminate polymer which may have remained in solution at $T_{c}$ and thus to prevent any further crys-

TABLE 1. Summary of crystallization procedure

$0.02 \%$ POM Solution $\longrightarrow$ First Crystallization $180^{\circ} \mathrm{C}(1 / 2 \mathrm{~h})$ $120^{\circ} \mathrm{C}(1 \mathrm{~h})$

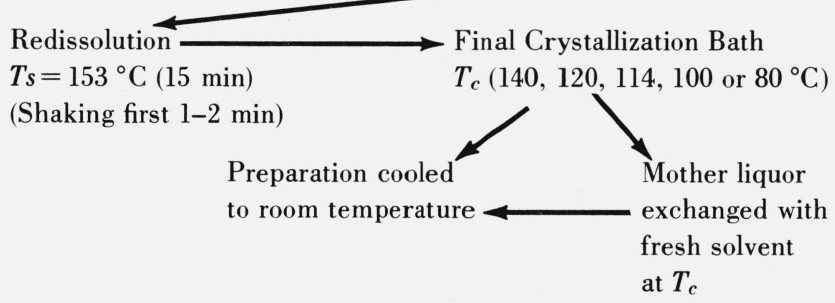


tallization during the final cooling of the preparation to room temperature. The exchange of the mother liquor with fresh solvent was carried out as follows using a filtration vessel akin in design to one previously described by Bassett and Keller [11]: Each preparation was rapidly transferred from the bath at $T_{c}$ and poured into the isothermally controlled filtration vessel maintained at $T_{c}$, this was followed by alternate filtration and addition of fresh preheated (to $T_{c}$ ) orthodichlorobenzene to the suspension of crystals, care being taken to ensure that the crystals were continuously immersed in liquid and not allowed to dry and form a mat between successive additions of fresh solvent to the suspension. Three exchanges of the liquid medium were carried out in each instance. After a final addition of fresh solvent to the suspension of crystals, the filtration vessel containing the latter suspension was allowed to cool to room temperature.

\subsection{Microscopy}

\section{(i) Optical Microscopy}

The "uncollapsed" shapes of the crystals were examined by observing them as they tumbled or were induced to tumble slowly while in suspension in liquid at room temperature under a microscope using phase contrast optics. Glass slides having a shallow central well in which droplets containing crystals in suspension were deposited, were used for this purpose. Because of the close match between the refractive indices of orthodichlorobenzene and POM, ${ }^{7}$ the crystals were only faintly perceptible in the latter medium. In order to distinctly perceive and examine the conformations of the crystals the orthodichlorobenzene in which they were in suspension was diluted with compatible liquids having appreciably higher or lower refractive indices. These liquids were added to the suspension of crystals in orthodichlorobenzene previously placed in the well of the slide, and the well was then covered with a thin coverglass. Several liquids covering a range of refractive indices were used including ethyl alcohol (refractive index $n_{D}=1.3611$ at $20^{\circ} \mathrm{C}[12]$ and methylene iodide $n_{D}=1.7559$ at $\left.10^{\circ} \mathrm{C}[12]\right)$. Dilution with the latter liquid was found most convenient for recording and exhibiting the conformational characteristics of the crystals photographically. The optical micrographs shown in Section 3 depict the crystals in suspension in orthodichlorobenzene diluted with methylene iodide. ${ }^{8}$

\footnotetext{
${ }^{7}$ The refractive index of orthodichlorobenzene is $n_{D}=1.5485$ at $20{ }^{\circ} \mathrm{C}[12]$. The following values have been reported by Jaffe and Wunderlich [13] for the refractive indices of light vibrating parallel to and perpendicular to the fiber axis in POM fibers produced by polymerizing trioxane in the solid state: $n_{11}=1.553$ (highest value observed) and $n_{\perp}=1.489$; $\bar{n}=\left(2 n_{\perp}+n_{11}\right) / 3=1.510$. It is not our intention to dwell here on the extensive literature concerning the nature of the orientation of the chain molecules in the fibrous products of the solid state polymerization of trioxane. It may be pointed out that preferential orientation of the chain molecules parallel to the fiber axis in the "extended chain crystal" samples tion of the chain mes used for the above mentioned refractive index measure "extend that $n_{11}>n_{\perp}$ : It should however be noted in this connection that instances of the occurrence of pseudo-twinned and/or twinned regions (differentiated as W "twinned" and T twinned
respectively by Colson and Reneker [14]), within which the orientation of the chain molecules deviates from the fiber axis direction in the constituent fibrous products of the solid state polymerization of trioxane, have been observed by means of electron diffraction $[7,14]$. An extensive bibliography and discussion of this topic may be found in a recently reported investigation [14].

${ }^{8}$ The methylene iodide used was supplied by the manufacturer with copper metal im. mersed in the liquid to enhance its stability.
}

(ii) Electron Microscopy

For the examination of the crystals in the electron microscope droplets of each preparation containing crystals in suspension in orthodichlorobenzene were deposited on electron microscope grids covered with a thin carbon film. The solvent was allowed to evaporate at room temperature after which the dried crystals were either examined as such in the electron microscope or were first shadowed with a platinum-palladium alloy $(80 \% \mathrm{Pt} / 20 \% \mathrm{Pd})$. The electron microscope used in this study was a JEM $6 a^{6}$ which was operated at 80 $\mathrm{Kv}$ using the double condenser system.

POM undergoes molecular scission and decomposes into volatile products upon irradiation with an electron beam. Individual unshadowed crystals have been observed to vanish completely within a very short period after exposure to the electron beam at the operating intensities normally used for clear visual observation of the images on the fluorescent screen of the electron microscope [5]. Shadowed crystals undergo similar decomposition, but the disposition of the shadowing metal about each crystal before irradiation remains unchanged however after the polymer has been volatilized and one is left with a direct replica of the lateral profile and topography of the original crystal as deposited on the carbon substrate [4]. Figures 5, 13, 19, 20, 21, 38 in Section 3 are such "replicas". The remaining bright-field or dark-field electron micrographs of shadowed or unshadowed crystals shown in Section 3 were recorded at very low beam intensities. Under these conditions the crystals and their diffraction contrast characteristics were preserved long enough (a few minutes) for their images to be recorded photographically. The bright field images were recorded using an objective aperture which passed the central beam and masked all the $\{h k i 0\}$ diffraction spots at the back focal plane of the objective lens.

\section{Observations on the Morphologies of Poly- oxymethylene Crystals}

\subsection{General}

A description is presented in ensuing subsections of the outcome of a combined optical and electron microscopical examination of the habits exhibited by the lamellar crystals of POM grown from the solutions prepared in the manner described in Section 2.1 when these solutions were transferred to baths held at one of the following temperatures, namely $T_{c}^{\circ}=140,120$, 114,100 , and $80{ }^{\circ} \mathrm{C}$. By way of a brief introduction, which serves to set the body of morphological data derived from the present study in perspective relative to the previous studies summarized in Section 1 , it may be pointed out in anticipation of Sections 3.2 to 3.4 that the lateral modes of propagation of the lamellar crystals formed in the various preparations examined in the present study varied as follows: At $T_{c}=140{ }^{\circ} \mathrm{C}$ the lamellae were six-sectored and exhibited the familiar hexagonal habit depicted in figure la; at $T_{c}=120^{\circ} \mathrm{C}$ lamellar crystals exhibiting an SS mode 
of lateral propagation akin to that illustrated in figure lb were formed; in the case of the $T_{c}=114^{\circ} \mathrm{C}$ preparations, SD lamellar crystals exhibiting a dendritic lateral habit akin to that shown in figure ld were formed, whereas in the case of the $T_{c}=100$ and $80^{\circ} \mathrm{C}$ preparations dendritic crystals were formed whose lateral growth involved the generation of new sectored outgrowths about the entire periphery of the basal lamella.

\subsection{Crystals Formed at $T_{c}=140{ }^{\circ} \mathrm{C}$}

(i) Preliminary Comments

At this temperature individual preparations yielded both the familiar monolayered six-sectored lamellar crystals bound laterally by $\{10 \overline{1} 0\}$ growth faces as well as multilayered crystals whose constituent lamellae were also six-sectored and bound laterally by $\{10 \overline{1} 0\}$ faces. Apart from some negligible effects associated with the different after-treatment procedures to which the preparations were subjected after crystallization at $140^{\circ} \mathrm{C}$, the populations of monolayered and multilayered crystals formed in different $T_{c}=140{ }^{\circ} \mathrm{C}$ preparations were indistinguishable from one another insofar as the habits of the crystals were concerned.

Observations concerning some effects associated with the two after-treatment procedures to which the preparations were subjected after having been maintained at $T_{c}=140^{\circ} \mathrm{C}$ for 24 hours will first be presented below. This will be followed by a consideration of observations pertaining to the conformation of monolayered crystals, after which salient characteristics exhibited by multilayered crystals will be described.

(ii) Effects Associated With the Different After-Treatment Procedures to Which the Preparations Were Subjected

In the case of preparations which had been kept at $140{ }^{\circ} \mathrm{C}$ for 24 hours and were then removed from the crystallization bath and allowed to cool to room temperature under ambient conditions, an examination of the crystals in the electron microscope revealed that the lamellae were bordered laterally by a very narrow strip which was thinner than the interior of the lamellae indicating that it resulted from further growth which the crystals underwent upon cooling to room temperature due to crystallization of polymer which had remained in solution in the mother liquor at $140^{\circ} \mathrm{C}$. The occurrence of this additional growth upon cooling the $T_{c}=140{ }^{\circ} \mathrm{C}$ preparations without filtration is illustrated in figure 2. A view of a hexagonal monolayer crystal formed in such a preparation is shown in figure $2 \mathrm{a}$. A view at higher magnification of the lower right hand corner of the same crystal is shown in figure $2 b$ in which a thinner outer strip bordering the periphery of the crystal may be discerned (see barbed arrow). Evidently the extent of the additional lateral growth represented by this strip is minimal compared to the lateral dimensions attained by the crystal at $140{ }^{\circ} \mathrm{C}$.

In the case of preparations which were subjected, at $140{ }^{\circ} \mathrm{C}$, to the filtration and dilution (washing) pro- cedure described in Section 2.1, the effect was that in addition to eliminating polymer which had remained in solution in the mother liquor at $140{ }^{\circ} \mathrm{C}$, this procedure caused a very mild and interestingly selective etching of the chain-folded lamellae. Although barely perceptible in low magnification electron micrographs such as those of the crystals shown in figure 3 and figure 4 this etching is clearly seen at high magnifications as illustrated in figure 5 in which portions of two contiguous sides of a monolayered crystal and portions of the corresponding adjoining sectors which they subtend are shown. The crystal from which this micrograph was obtained had been shadowed before it was exposed to the electron beam. The barbed arrow in figure 5 points in the direction of shadowing. The micrograph was recorded after the polymer had "vaporized" under the influence of the electron beam leaving behind an exact replica (see Section 2.1) of the original crystal. The occurrence of minute voids (see unbarbed arrows) ${ }^{9}$ resulting from the selective etching of polymer in the interior of the lamella during filtration and washing can be readily discerned in figure 5. These voids which are apparently randomly dispersed about the interior of the lamella are characteristically longer than they are wide. The lengths

${ }^{9}$ Note that the elongated features (voids) pointed to by the unbarbed arrows are distinctly lighter (absence of evaporated metal) on the side closest to the origin of shadowing and much darker (accumulation of metal) on the side further away from the origin of shadowing; this clearly indicates that these features are voids and not ridges.

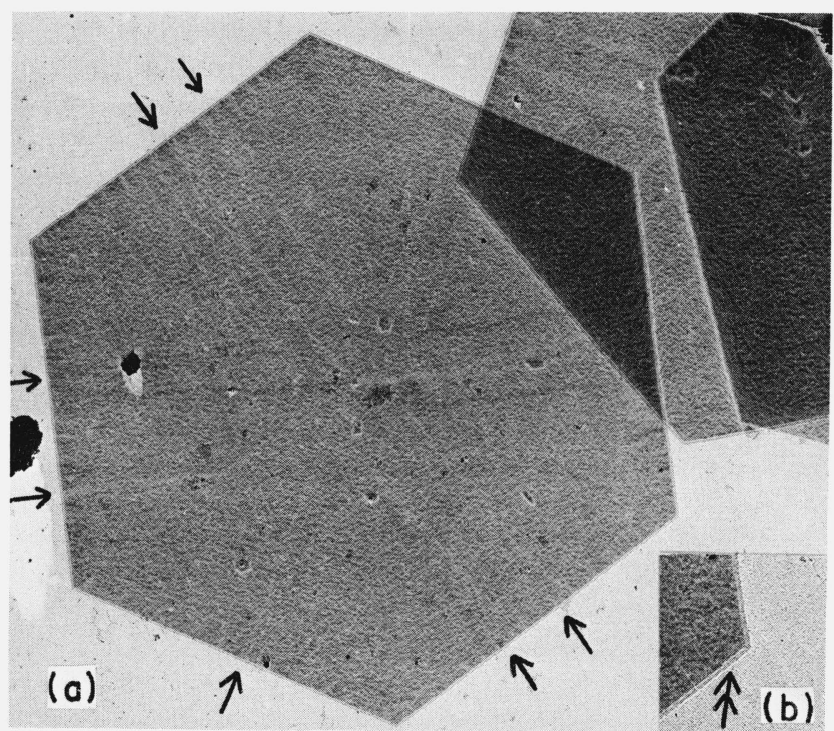

FIGURE 2. (a) Electron micrograph of a monolayer crystal formed in a $\mathrm{T}_{\mathrm{c}}=140{ }^{\circ} \mathrm{C}$ preparation which had not been subjected to filtration and washing at $140{ }^{\circ} \mathrm{C}$ prior to cooling to room temperature.

Unbarbed arrows point to streaks running at right angles to outer edges of the crystal (see text, section 3.2 (iii)). $\mathrm{Pt} / \mathrm{Pd}$ shadowed. X3500.

(b) A view at higher magnification of lower right hand corner of the same crystal showing thin outer strip (see barbed arrow) formed upon cooling preparation to room temperature.

$\mathrm{Pt} / \mathrm{Pd}$ shadowed, $\mathrm{X} 6900$. 


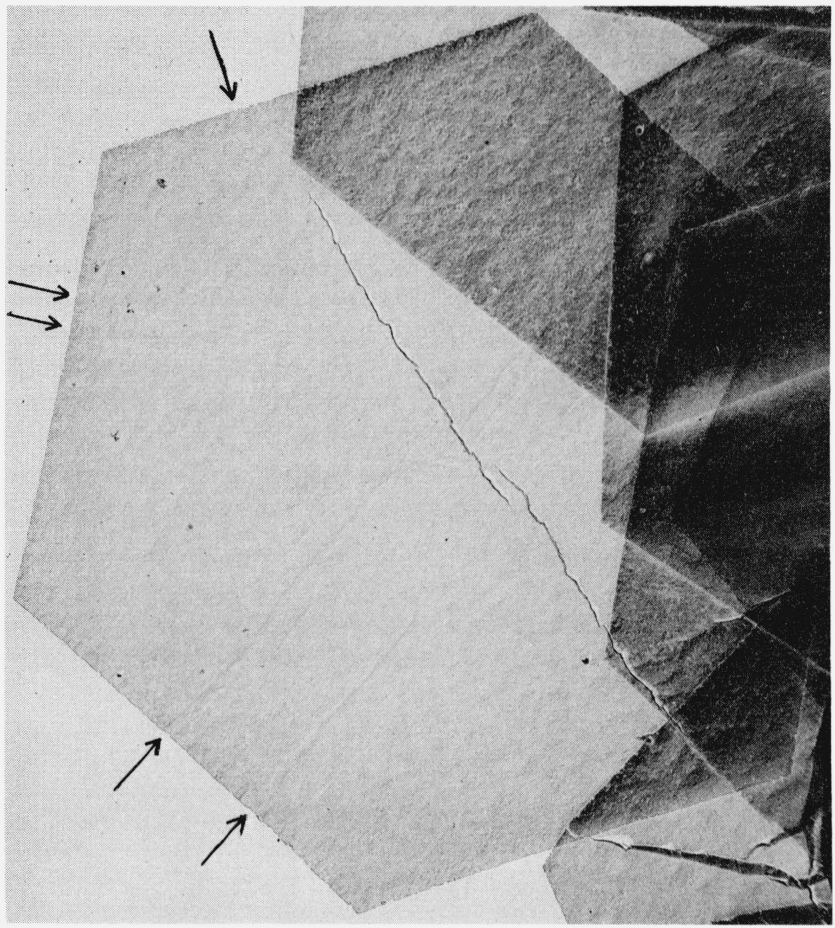

FIGURE 3. Electron micrograph of a monolayer crystal formed in a $\mathrm{T}_{\mathrm{c}}=140{ }^{\circ} \mathrm{C}$ preparation which had been subjected to filtration and washing at $140^{\circ} \mathrm{C}$ prior to cooling to room temperature.

Arrows point to streaks running at right angles to the outer edges of the crystal (see text sec. 3.2 (iii)). $\mathrm{Pt} / \mathrm{Pd}$ shadowed. $\mathrm{X} 4300$.

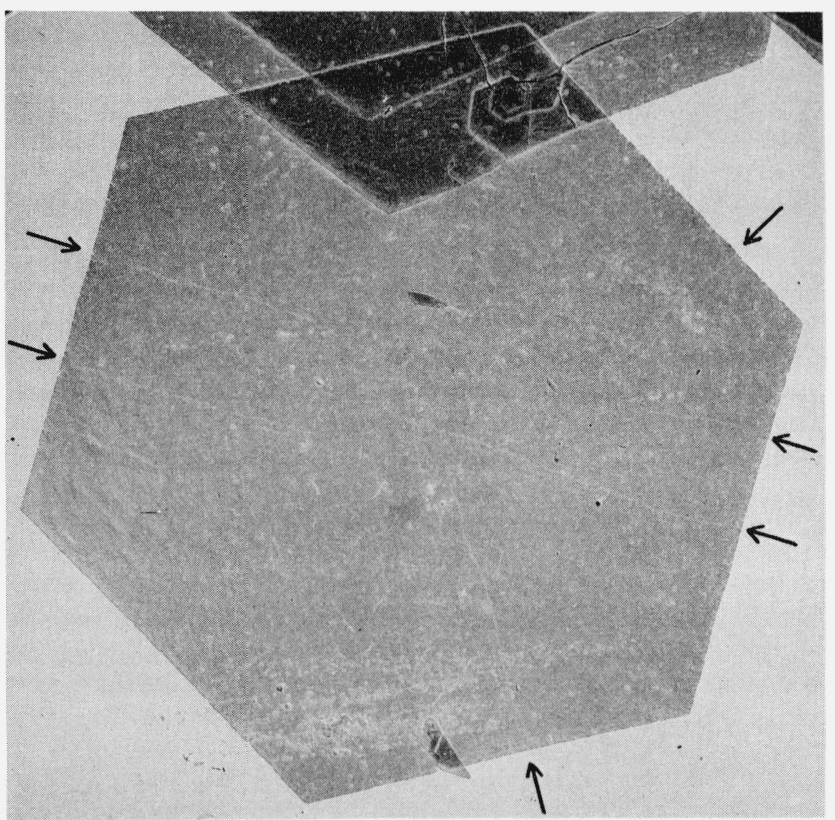

FigurE 4. Electron micrograph of a monolayer crystal formed in $a \mathrm{~T}_{\mathrm{c}}=140{ }^{\circ} \mathrm{C}$ preparation which had been subjected to filtration and washing at $140^{\circ} \mathrm{C}$ prior to cooling to room temperature.

Arrows point to streaks running at right angles to the outer edges of the crystal (see text, sec. 3.2 (iii)). $\mathrm{Pt} / \mathrm{Pd}$ shadowed. X4000.

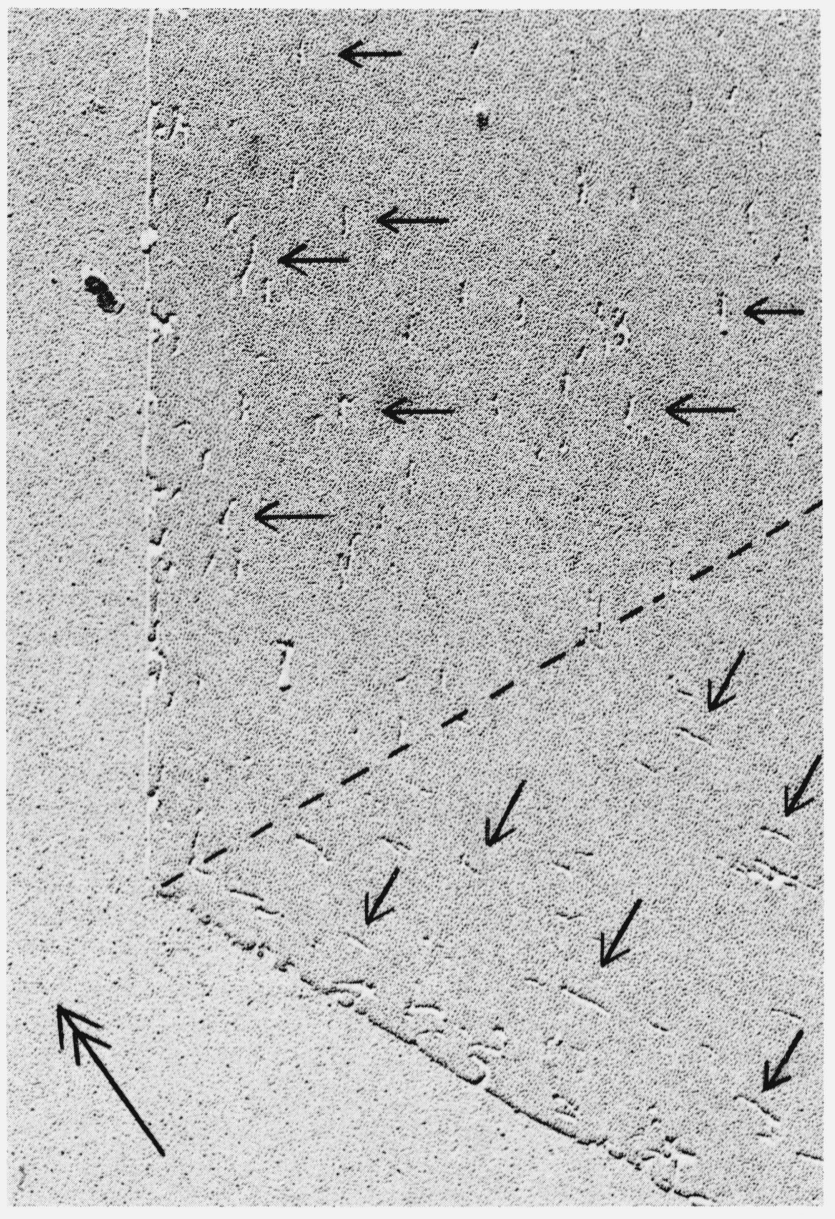

FiguRe 5. Electron micrograph of a corner and adjoining portions of a six-sectored monolayered crystal formed in a $\mathrm{T}_{\mathrm{c}}=140^{\circ} \mathrm{C}$ preparation which had been subjected to filtration and washing at $140{ }^{\circ} \mathrm{C}$ prior to cooling to room temperature.

This image of a shadowed crystal was recorded after the polymer had vaporized under the influence of the electron beam. Dashed line denotes boundary between the adjoining pair of sectors subtended by the two contiguous $\{10 \overline{1} 0\}$ crystal edges portions of which are visible in the field of view. Barbed arrow denotes direction of shadowing. Unbarbed arrows in the interior of each sector point to some of the numerous elongated voids which are oriented with their longer dimension preferentially parallel to the outer $\{10 \overline{1} 0\}$ edge which subtends the particular sector in which they are situated. $\mathrm{Pt} / \mathrm{Pd}$ shadowed. X33700.

of the discernible voids in figure 5 vary in the range $0.03 \mu \mathrm{m}$ to $0.16 \mu \mathrm{m}$ and they are approximately 0.016 $\mu \mathrm{m}$ wide. In addition, as may be seen from a consideration of the voids situated on opposite sides of the dotted line (which corresponds to the sector boundary) in figure 5 , the voids are characteristically oriented with their longer dimension preferentially parallel to the outer edge $(\{10 \overline{1} 0\}$ growth face) which subtends the particular sector in which they are situated, thus attesting to the preferential etching of polymer in a direction parallel to the $\{10 \overline{1} 0\}$ planes along which the chain molecules are folded in each sector. This directionally selective etching in the constituent sectors is certainly consistent with the six-sectored nature of these hexagonal-shaped lamellae of POM, indeed it provides in itself further substantiation that such lamellae consist of six distinguishable fold domains. 
The nature of the dispersed sites at which this selective etching occurs preferentially in each sector remains undetermined at present. Finally, as may also be seen in figure 5 , the slightly "nibbled" appearance exhibited by the otherwise straight lateral edges of the lamella indicates that some etching also occurred at its periphery during filtration and washing.

\section{(iii) Observations Pertaining to the Conformation of the Hexagonal Shaped Monolayered Crystals}

A description will first be presented in this section of the outcome of a direct examination of the conformation of the crystals in suspension in liquid as seen under the optical microscope. The observations derived from this optical microscopical study will then be considered in the light of the various views, summarized in Section 1, which have been previously advanced concerning the possible origins of the nonplanar character of six-sectored lamellae of POM. Some conclusions concerning both the origins and nature of the nonplanar character of the crystals obtained in the present study will be subsequently advanced and discussed in association with various features exhibited by the crystals in the electron microscope. These latter features, which bear on the nonplanar character of the crystals, have been variously observed in different previous studies [4-8].

Four optical phase contrast micrographs representing different views of a monolayered single crystal (same unfiltered preparation as that of crystal shown in fig. 2) in suspension in liquid are shown in figure 6 . Figures $6 \mathrm{a}$ to $6 \mathrm{~d}$ were recorded sequentially as the crystal tumbled extremely slowly in the liquid. Figure 6a represents the lamella as viewed edge-on, i.e., with its central axis of six-fold symmetry lying parallel to the field of view, whereas in figure $6 \mathrm{~d}$ this central axis is oriented at right angles to the field of view. Figures $6 \mathrm{~b}$ and $6 \mathrm{c}$ show the same crystal at successive stages in its change in orientation from that shown in figure $6 \mathrm{a}$ to that shown in figure 6d. An edge-on view of a monolayered crystal from a filtered preparation (same preparation as the crystals in figs. 3,4 ) is shown in figure 7.

Figures $6 \mathrm{a}$ and 7 serve to illustrate a characteristic feature of the hexagonal shaped monolayered crystals formed at $T_{c}=140^{\circ} \mathrm{C}$. namely they exhibit an essentially linear profile when viewed edge-on, ${ }^{10}$ thus indicating that any departure from planarity in the shape of these crystals is only slight. The problem is how slight? Previous views which have been advanced concerning the possible origins of the nonplanar character of hexagonal six-sectored lamellae of POM provide a framework of reference which serves as a basis for discussing this problem in comparative terms.

One possible source of nonplanarity which may be envisaged [4-7] is that the folds in successive $\{1010\}_{f}$ planes in each sector might be regularly staggered in a

${ }^{10} \mathrm{It}$ is noteworthy that this feature is exhibited by the monolayered crystals from both filtered and unfiltered preparations. In short, the mild etching which occurred in the lamellae formed in the preparations which had been subjected to a final filtration and wash. ing procedure at $140{ }^{\circ} \mathrm{C}$ did not apparently affect the conformation of the lamellae.
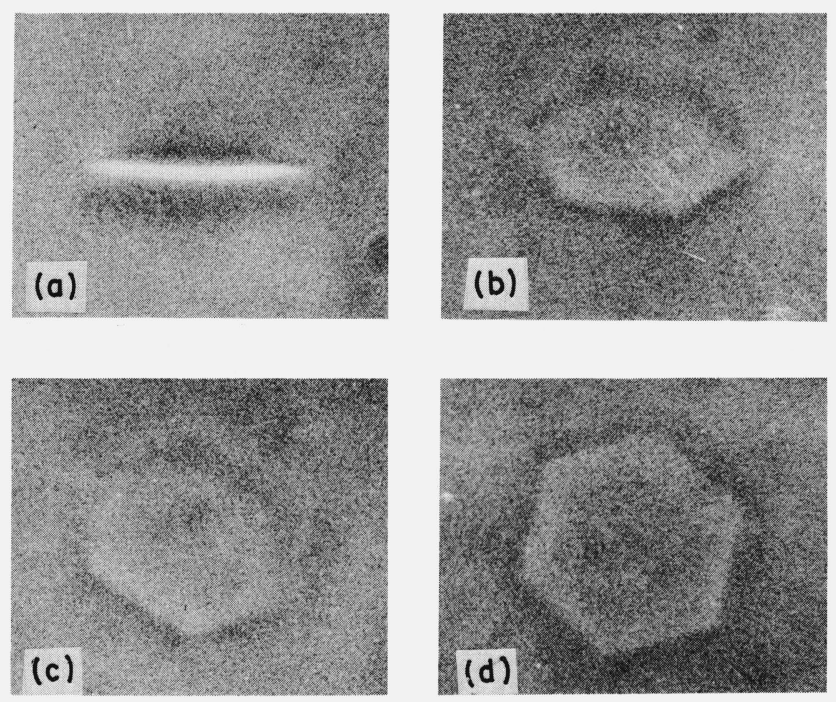

Figure 6. Sequence of optical micrographs of a monolayered lamellar crystal formed in a $\mathrm{T}_{\mathrm{c}}=140^{\circ} \mathrm{C}$ preparation which had not been filtered and washed prior to cooling to room temperature.

The crystal is depicted as seen consecutively in different perspectives as it was slowly tumbling while in suspension in liquid. (a) and (d) show the crystal as viewed edge-on and flat-on respectively; (b) and (c) depict the crystal at intermediate inclinations between the extremes shown in (a) and (b). Phase Contrast. X1300.

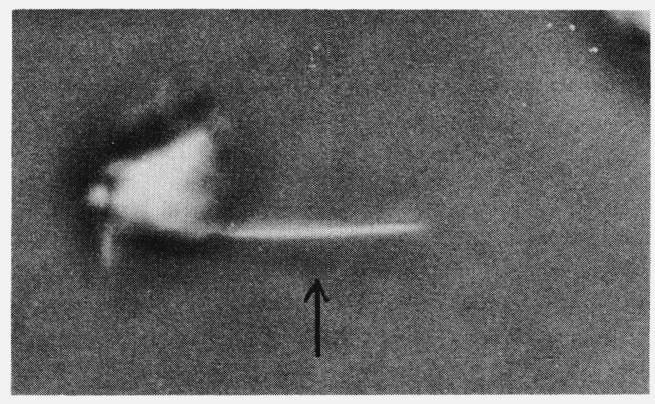

FIGURE 7. Optical micrograph showing an edge-on view of a monolayered lamellar crystal (arrow) formed in a $\mathrm{T}_{\mathrm{c}}=140^{\circ} \mathrm{C}$ preparation which had been filtered and washed prior to cooling to room temperature.

The crystal is in suspension in liquid. The structure on the left hand side of the micrograph is a complex multilayered crystal (see sec. 3.2 (iv)) situated at a different level in the liquid. Phase Contrast, X1000.

direction parallel to the $c$ axis. The following considerations may be raised regarding this suggestion. One chain in a 29/16 helical conformation passes through the metrically hexagonal unit cell of POM, the axis of the helix is parallel to the $c$ axis of the unit cell and the repeat distance per monomer unit along the chain axis is $1.93 \AA$ [9]. The oxygen (or carbon) atoms of successive monomer units in the helical chains of the POM crystal lattice lie in planes which are perpendicular to the $c$-crystallographic axis and are spaced $1.93 \AA$ apart in that direction. This feature restricts the magnitude of the regular stagger (if it did occur) between successive $\{10 \overrightarrow{10}\}_{f}$ planes in the constituent fold domains of six-sectored lamellae to a distance corresponding to the repeat distance of one monomer 
unit in the $c$ axis direction, i.e., $1.93 \AA$, or to whole number multiples thereof. It may be readily calculated that if a regular stagger of $1.93 \mathrm{~A}$ between the folds in successive $\{10 \overline{1} 0\}_{f}$ planes (whose interplanar spacing is $3.87 \AA$ ) in each sector did occur a hexagonal based hollow pyramidal crystal having an angle of elevation of $26.5^{\circ}$ (the angle between the base and the line joining the apex of the pyramid to the midpoint of one of its basal edges) would result. The essentially linear profile which the monolayered six-sectored crystals formed in the $T_{c}=140{ }^{\circ} \mathrm{C}$ preparations exhibit when viewed edge-on as shown in figures 6-7 argues against the incidence of such regular fold staggering in these crystals.

The other cause of nonplanarity which has been proposed is that six-sectored lamellae buckle slightly under the influence of stresses which result from the accommodation across fold domain boundaries of the asymmetric distortion of the POM unit cell which occurs, as shown by Bassett [5], in each fold domain. The observations and views of Bassett [5] and Bassett et al. [6] concerning this matter have been summarized briefly in Section 1. They bear to be reiterated and considered in somewhat more detail at this juncture. The basic feature of interest in this connection is the following fact derived by Bassett [5] from an analysis of Moiré patterns exhibited by bilayered crystals of POM, namely that the spacing between the $\{10 \overline{1} 0\}_{f}$ planes differs by approximately 0.32 percent from the spacing between the $\{10 \overline{1} 0\}_{n f}$ planes in each fold domain in six-sectored chain folded lamellae of POM. As a result of this distortion of the subcell in each domain, which distortion has been attributed to an asymmetric bulkiness of the chain folds, there results a lattice mismatch across the boundaries between adjacent fold domains in the lamella [5]. It was suggested that stresses resulting from the accommotion of this mismatch at the fold domain boundaries cause the lamella to buckle slightly. The following considerations pertaining to nature and magnitude of such buckling may be advanced.

It is not possible to distinguish between whether $d_{\{10 \overline{1} 0\}_{f}}>d_{\{10 \overline{1} 0\}_{n f}}$ or vice versa from Moiré patterns [5]. All that can be derived from the Moiré patterns is the magnitude of the difference between $d_{\{10 \overline{1} 0\} f}$ and $d_{\{10 \overline{10}\}_{n f}}$, one is therefore faced with two possibilities concerning the inequality between these interplanar spacings. Different conformations which it may be envisaged a six-sectoral lamella of POM might adopt as a consequence of this inequality in interplanar spacings depending on which of the spacings, $d_{\{10 \overline{10} f}$ or $d_{\{10 \overline{10}\}_{n f}}$ is the larger in the fold domains are discussed below with reference to figures 8 to 10 .

The dotted lines in figure $8 \mathrm{a}$ represent the outline of the periphery and the sector boundaries in an imagined planar six-sectored lamella in which $d_{\{10 \overline{1} 0\}_{f}}=d_{\{10 \overline{1} 0\}_{n f}}$ and in which the stems of the folded molecules are at right angles to the surface of the lamella. It may be calculated that if $d_{\{10 \overline{1} 0\}_{f}}>d_{\{10 \overline{1} 0\}_{n f}}$ by about 0.32 percent then each sector will be expanded in a direction

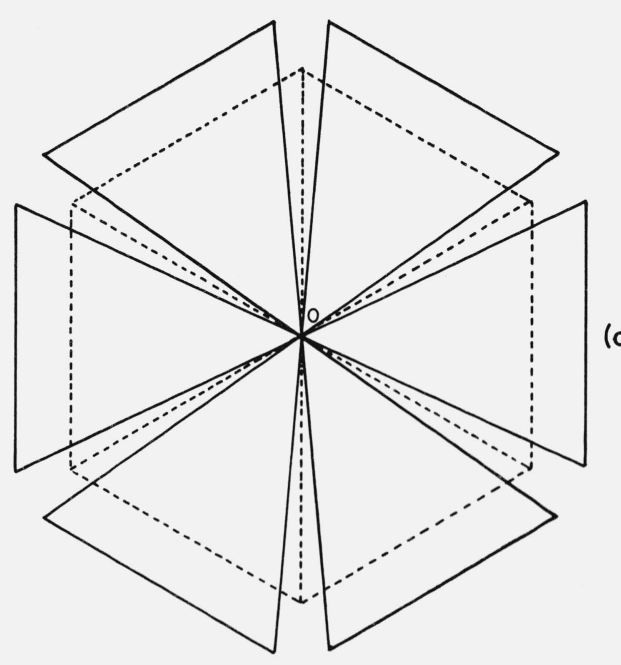

(a)

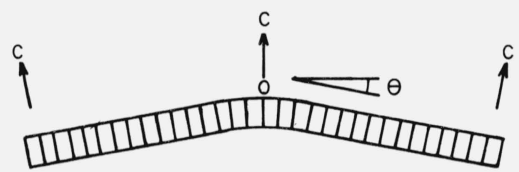

(b)

FigURE 8. (a) Schematic representation of an idealized six-sectored lamella in which $\mathrm{d}_{\{10 \overline{1} 0\}_{\mathrm{f}}}=\mathrm{d}_{\{10 \overline{1} 0\}_{\mathrm{nf}}}$ in each sector $(---)$, and distorted sectors in which $\mathrm{d}_{\{10 \overline{1} 0\}_{\mathrm{f}}}>\mathrm{d}_{\{10 \overline{1} 0\}_{\mathrm{nf}}}(-($ ). (b) Schematic representation of central cross-sectional view of two opposite sectors in a slightly conical six-sectored lamella.

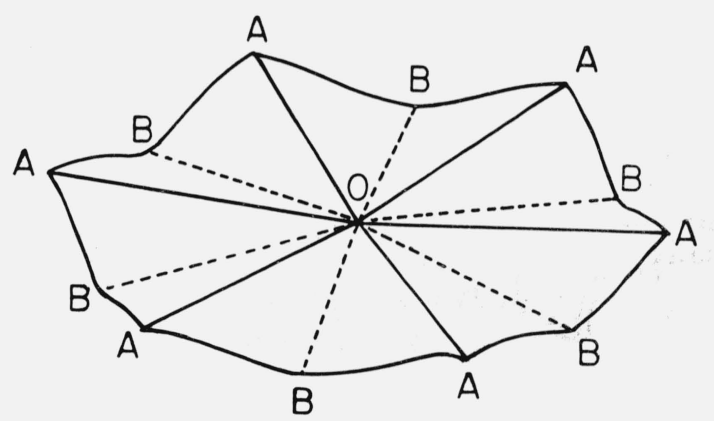

FIGURE 9. Three dimensional perspective of a possible six-sectored lamella having a six-fold symmetrical wavy conformation resulting from $\mathrm{d}_{\{10 \overline{1} 0\}_{\mathrm{f}}}<\mathrm{d}_{\{10 \overline{1} 0\}}$ in each sector.

Lettering corresponds to that in figure 10.

normal to its outer edge and that there will be an angular deficit of $12.6^{\prime}$ between each pair of adjacent sectors as shown in exaggerated fashion by the full lines in figure 8a. A reconstruction of the lamella from the distorted sectors such that there be continuity between sectors at the center $(\mathrm{O})$ as well as across sector boundaries may be accomplished by simultaneously lifting the sectors out of the plane of the diagram at $(\mathrm{O})$ where they are initially in contact. While this operation brings the sectors in lateral contact with one another, there would result in view of the thickness of the sectors, a V-shaped groove along the sector boundaries if the sectors were kept flat. This feature may be overcome by a bending or buckling of the individual sectors so that contact between 


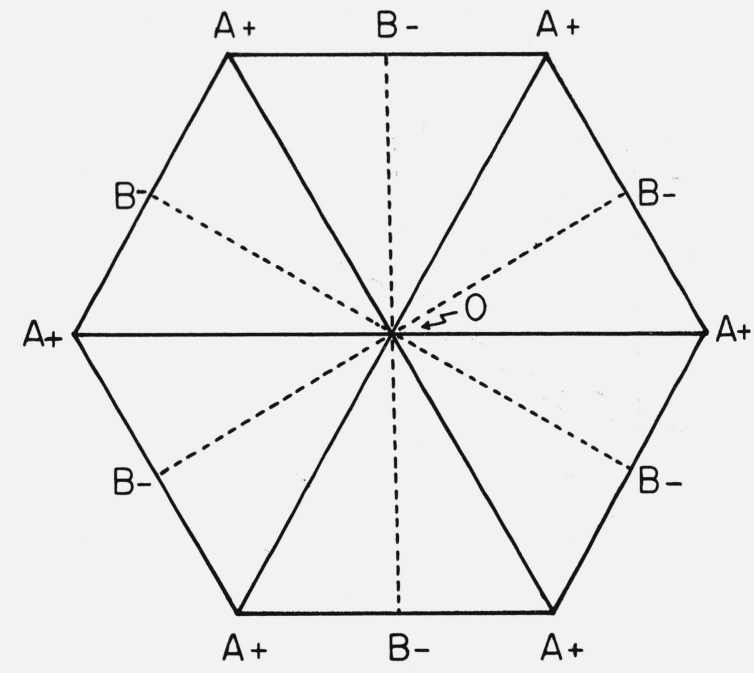

FigURE 10. Topographical 'map' of a six-sectored lamella such as that shown in figure 9 as seen along its central axis.

The center $(\mathrm{O})$ lies in the plane of the diagram whereas the corners $(\mathrm{A})$ and the midpoints (B) lie respectively above $(+)$ and below $(-)$ the plane passing through $(\mathrm{O})$ at right angles to the central axis of the lamella.

adjacent sectors is made throughout their thickness. By the same token continuity between the sectors at the apex $(\mathrm{O})$ would be maintained by a slight bending of the sectors at $(\mathrm{O})$. The gist of these considerations is that if $d_{\{10 \overline{1} 0\}_{f}}>d_{\{10 \overline{1} 0\}_{n f}}$ one might expect the buckled lamella to have an essentially conical shape and be slightly rounded at the apex $(\mathrm{O})$ as shown in figure $8 \mathrm{~b}$ which represents a cross-sectional view of two opposite sectors in the lamella. It is also envisaged that the stems of the folded molecules are oriented at right angles to the surface of the lamella throughout. Finally, it may be estimated (geometrically) on the basis of the 0.32 percent difference between $d_{\{10 \overline{1} 0\}_{f}}$ and $d_{\{10 \overline{1}\}_{n f}}$ that the angle of elevation $(\theta)$ of the conical lamella would be about $4.5^{\circ}$.

Turning to the case where $d_{\{10 \overline{1} 0\}_{f}}<d_{\{10 \overline{1} 0\}_{n f}}$ the application of a 0.32 percent difference between these spacings in each sector will result in an excess of $12.6^{\prime}$ from the ideal of $60^{\circ}$ in the angle subtended by the outer edge of each sector at the center of the lamella. This angular excess may be accommodated by bending or buckling of the sectors, we can imagine that in this situation the lamella might adopt a six-fold symmetrical wavy conformation such as that illustrated in figure 9, the stems of the folded molecules being normal to the surface of the lamella throughout. A topographical map of such a crystal is depicted in figure 10 which represents a projection of the crystal as seen along its central axis. The lettering in figure 10 corresponds to that in figure 9 . The center $(\mathrm{O})$ of the crystal lies in the plane of the diagram. The plus and minus signs in figure 10 which are assigned to the corners (A) of the lamella and the midpoints (B) of the outer edges of the sectors respectively, serve to indicate that points (A) lie above and points (B) lie below the plane of the diagram and hence the center $(0)$. It may be estimated (geometrically) that angle of inclination of the line joining $A B$ in each sector relative to the plane of reference passing through the center of the crystal $(0)$ at right angles to its central axis would be approximately $5^{\circ}$ and that the angle of inclination of the line joining $\mathrm{O}$ to $\mathrm{B}$ relative to the same plane of reference would be about $1.5^{\circ}$.

Simplified as the considerations advanced in the preceding two paragraphs may be, it is fairly evident that departures from planarity resulting solely from buckling due to the incidence of an asymmetric distortion of the unit cell in fold domains would be substantially smaller than would be the case if regular fold staggering occurred, and too small to be established unequivocally and determined quantitatively from an examination of the crystals in suspension in liquid under the optical microscope. (Sinuous shapes of greater complexity than those discussed with reference to figure 8 and figures 9 and 10 may also be envisaged).

In summary the optical microscopical observations described above strongly suggest that regular fold staggering did not occur in the monolayered sixsectored crystals formed in the $T_{c}=140^{\circ} \mathrm{C}$ preparations in the present study and that, borrowing in part from Bassett [6], any departure from planarity in the crystals is due to slight buckling associated with the asymmetric distortion of the subcell in the fold domains. The fact that the crystals are indeed not planar in their original as-grown state is reflected by several features which they exhibit when examined in the electron microscope. These features are described below where they are discussed (in conjuction with the 'models' depicted in figures 8, 9, and 10).

Faint streaks of varying lengths which run at right angles to the edges of the crystals may be seen in the bright field electron micrographs of the monolayered crystals shown in figure 2 (crystal from an unfiltered preparation) and figures 3 and 4 (crystals from a filtered preparation) in which it may also be seen that these streaks, which are pointed to by unbarbed arrows, are confined within the borders of the constituent sectors in the crystals, i.e., they do not cross over from one sector to an adjacent one, and that furthermore they exhibit either brighter or darker contrast relative to the surrounding portions of the sector in which they are situated. The occurrence of these streaks is a manifestation of diffraction contrast corresponding to variations in the orientation of the stems of the folded molecules (i.e., the $c$ axis of the POM subcell) within the constituent sectors in these sedimented crystals relative to their surface. This latter feature is also evidenced in $\{10 \overline{1} 0\}$ dark field images obtained from such crystals, as indicated below.

A dark field electron micrograph obtained from a crystal formed in the same unfiltered preparation as that in figure 2 is shown in figure 1la. A diagram of a six-sectored lamella in which the sectors are identified as $s_{1}$ to $s_{6}$ to correspond to the sectors in the crystal shown in figure $11 \mathrm{a}$, is depicted in figure $11 \mathrm{~b}$ as an aid to the description of the features exhibited in figure 

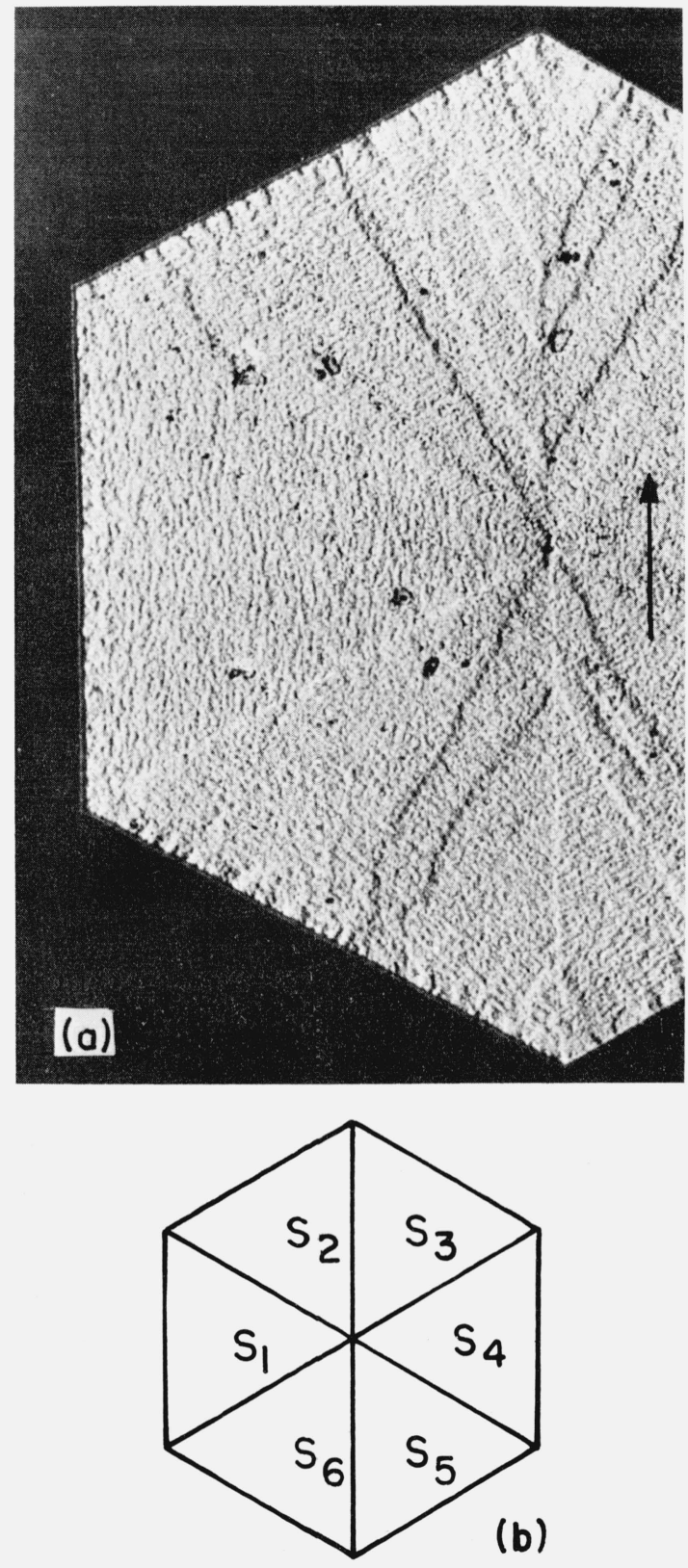

FIGURE 11(a). $\quad\{10 \overline{1} 0\}$ dark field electron micrograph of a monolayered crystal formed in a $\mathrm{T}_{\mathrm{c}}=140{ }^{\circ} \mathrm{C}$ preparation which had not been filtered and washed prior to cooling to room temperature.

This image was formed using the diffraction spot from the $\{10 \overline{1} 0\}$ planes in the crystal which are parallel to the vertical arrow in the picture; X5300.

(b) Schematic representation of a six-sectored crystal with sectors identified, see text.

11a. The $10 \overline{1} 0$ diffraction spot which was used to form the image shown in the latter figure corresponds to the $\{1010\}$ planes in the crystal which run parallel to the vertical arrow shown in figure 1la; these planes are fold planes in sectors $s_{1}$ and $s_{4}$ but not so in the other four sectors. As may be seen in figure 11a streaks which are either darker or brighter than the overall background intensity exhibited by the crystal and which run at right angles to the crystal edges may be seen in the sectors in which the $\{10 \overline{1} 0\}$ planes which are imaged are not fold planes (sectors $s_{2}, s_{3}, s_{5}, s_{6}$, figure 11b), no such streaks occur however in the two remaining sectors $\left(s_{1}\right.$ and $\left.s_{4}\right)$. A similar systematic absence of streaks in the pair of opposite sectors in which the $\{10 \overline{1} 0\}$ planes which are imaged are the $\{10 \overline{1} 0\}_{f}$ planes, which are parallel to the crystal edges which subtend the sectors, has been observed in dark field images by Bassett [5]. On the basis of this systematic absence Bassett [5] and Bassett et al. [6] concluded that the streaks are a consequence of and correspond to the occurrence in each sector of a slight tilting of the crystal lattice relative to the surrounding portions of the sector about an axis normal to the $\{10 \overline{10}\}$ growth face which subtends each sector. The incidence of such localized tilting was considered to occur when the crystals are sedimented and dried on a flat substrate $[5,6]$ and was adduced as evidence that the crystals are not flat as grown.

It may be noted that apart from the incidence of the aforementioned streaks, short range variations in brightness occur in all the sectors which exhibit as a consequence a somewhat "grainy" appearance as may be seen in figure 1la. It should be borne in mind in this connection that the lamella shown in figure 11a is a crystal from a preparation which had not been filtered and diluted prior to being cooled to room temperature from $140{ }^{\circ} \mathrm{C}$, thus there are no small etched voids in the crystal such as those which occur, as pointed out earlier, in crystals from preparations which had been filtered and diluted at $140^{\circ} \mathrm{C}$. In short, the possibility of artifacts resulting from etching does not arise in connection with above mentioned "granularity" which has also been observed by Garber and Geil [8]. The fact that this feature is also manifested in sector $s_{1}$ in which the $\{10 \overline{1} 0\}$ planes which are imaged are the $\{10 \overline{1} 0\}_{f}$ planes which are parallel to the $\{10 \overline{1} 0\}$ growth face which subtends that sector may be interpreted as indicating that there occur within each sector of the crystal in its sedimented state small short range variations in the orientation of the $\{10 \overline{1} 0\}_{f}$ planes relative to the incident beam due to the localized incidence of tilting within each sector about a direction parallel to the corresponding $\{10 \overline{1} 0\}$ growth face, or at any rate about directions having a component vector parallel to that face. It is not unreasonable to expect that such misorientations would be manifested in a nonplanar crystal when it collapses on a flat substrate. It seems from a close examination of the sectors that the variations in brightness associated with these misorientations do not occur in an entirely random fashion about each sector, rather there is a suggestion that the regions of darker contrast occur as narrow fuzzy lines which traverse each sector along paths (of varying lengths) which meander along a direction parallel to the outer edge of the sector.

In addition to the effects due to diffraction contrast which have been described in the previous three paragraphs, which effects as has been pointed out evidence 
in themselves the nonplanar as-grown character of the six-sectored lamellae, this latter feature is further substantiated by the occurrence in some crystals of slight pleats, (such as that which can be seen in the crystal shown in figure 3 ) resulting from the collapse of the crystals.

In summary we find that whereas the electron microscopical observations indicate that the monolayered six-sectored crystals formed in our $\mathrm{T}_{c}=140{ }^{\circ} \mathrm{C}$ preparations are not flat as grown, the optical microscopical observations on the crystals (as viewed in suspension in liquid) argue against the incidence of regular fold staggering, and favor the view that the nonplanar character of the crystals is due to slight buckling associated with the asymmetric distortion of the subcell in the fold domains due to the bulkiness of the chain folds. The question as to whether the as-grown shape of the slightly buckled crystals corresponds to or approximates that considered earlier with reference to figure $8\left(d_{\{10 \overline{1} 0\}_{f}}>d_{\{10 \overline{1} 0\}}\right)$ or that depicted in figures 9 and $10\left(d_{\{10 \overline{1}\}_{f}}<d_{\{10 \overline{1} 0\} n f}\right)$ remains uncertain however. The localized misorientations of the lattice about individual sectors, which misorientations result from tilting either about an axis perpendicular to or an axis parallel to the corresponding $\{10 \overline{1} 0\}$ growth face (both types of tilt apparently occur), and the incidence of pleating, do not lend themselves to an unequivocal "reconstruction" of the uncollapsed shape of the crystals. The matter of the conformation of these crystals will be referred to again in the general discussion presented in Section 4. It suffices for the present to note that the six-sectored monolayered crystals formed in the $T_{c}=140{ }^{\circ} \mathrm{C}$ preparations are not perfectly flat, and that their departure from planarity is apparently only slight and not inconsistent with the possible slightly buckled conformations considered earlier with reference to figures 8-10.

(iv) Multilayered Crystals Formed at $\boldsymbol{T}_{c}=140^{\circ} \mathrm{C}$

As pointed out in Section 3.2 (i) both monolayered crystals such as those described in Section 3.2 (ii) and 3.2 (iii) as well as numerous multilayered crystals whose constituent lamellae are bound laterally by $\{10 \overline{1} 0\}$ growth faces are formed in individual preparations at $T_{c}=140{ }^{\circ} \mathrm{C}$. The optical and electron micrographs shown in figures 12-15 (filtered preparation) serve to illustrate the diversity in the character of these multilayered crystals which may in essence be divided into two categories, namely, simpler ones whose constituent chain folded lamellae are apparently stacked parallel or essentially parallel to one another, and a variety of more complex ones in which the constituent lamellae are not coplanar. The latter crystals are "axialitic" (see later) in character.

No appreciable splaying is observed between the constituent chain-folded lamellae in the simpler multilayered crystals. When viewed edge-on while in suspension in liquid these crystals exhibit an overall essentially linear profile as may be seen in figure 12. This type of crystal may be identified with crystals (from the same preparation) such as crystal (A) shown in figure 13 which does not exhibit in the sedimented state any pronounced collapse features or deformation, in contrast with crystals (B) and (C) in figure 13 and the crystal shown in figure 14. Crystal (A) in figure 13 is composed of superimposed chainfolded layers which have developed in a spiral-like fashion through the agency of screw dislocations located at or near the center of the basal lamella. It may also be noted that successive layers in the crystal

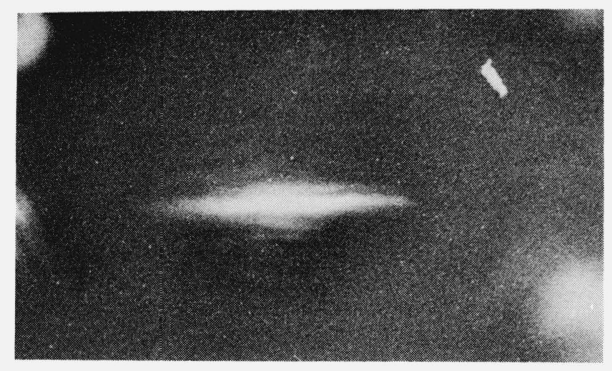

FIGURE 12. Optical micrograph showing an edge-on view of a multilayered crystal formed in a $\mathrm{T}_{\mathrm{c}}=140{ }^{\circ} \mathrm{C}$ preparation which has been filtered and washed at $140{ }^{\circ} \mathrm{C}$ prior to cooling to room temperature.

The crystal is in suspension in liquid. Phase contrast, X1400.

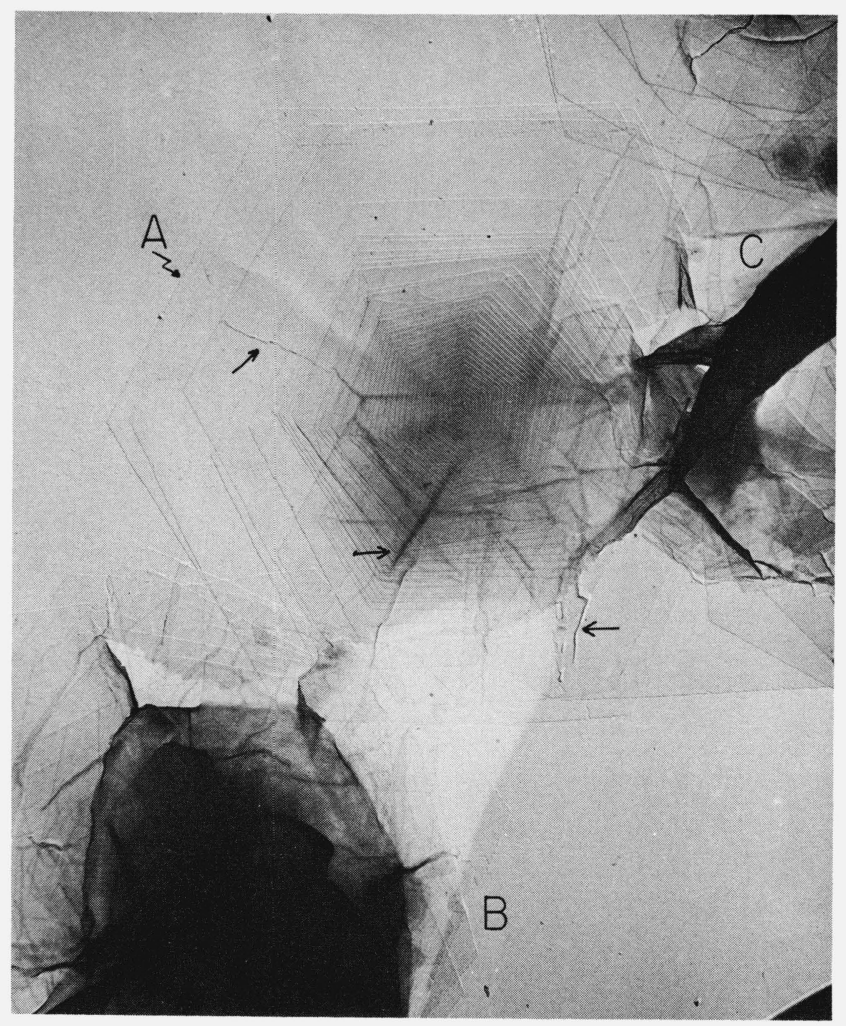

FIGURE 13. 'Replica' of multilayered crystals formed in a $\mathrm{T}_{\mathrm{c}}=$ $140{ }^{\circ} \mathrm{C}$ preparation which had been filtered and washed at $140{ }^{\circ} \mathrm{C}$ prior to cooling to room temperature.

Image recorded after prolonged exposure to the electron beam [11], see text. $\mathrm{Pt} / \mathrm{Pd}$ shadowed, X3200. 


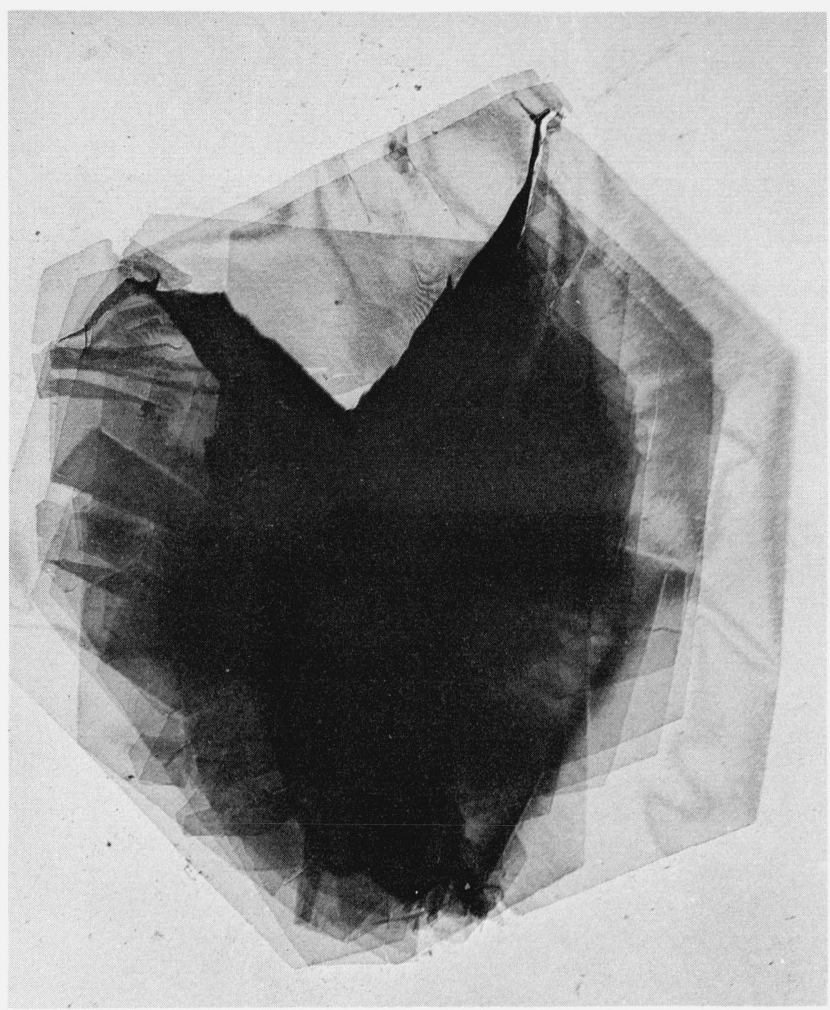

FIGURE 14. Electron micrograph of a complex multilayered crystal formed in a $\mathrm{T}_{\mathrm{c}}=140{ }^{\circ} \mathrm{C}$ preparation which had been filtered and washed prior to cooling at room temperature.

$\mathrm{Pt} / \mathrm{Pd}$ shadowed, $\mathrm{X} 4100$.

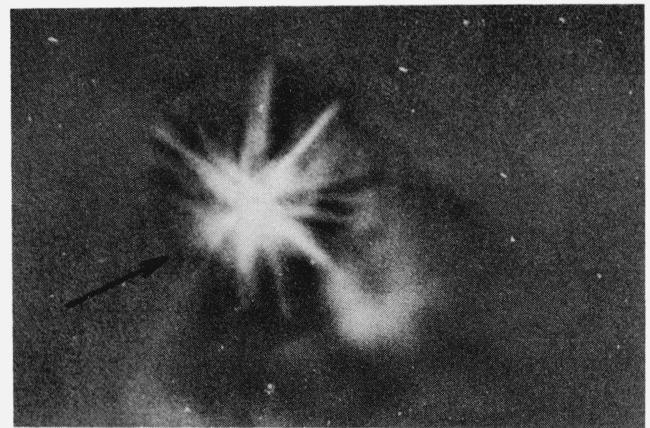

FigURE 15. Optical micrograph of complex 'axialitic' multilayered crystal (arrow) formed in a $\mathrm{T}_{\mathrm{c}}=140{ }^{\circ} \mathrm{C}$ preparation which had been filtered and washed at $140^{\circ} \mathrm{C}$ prior to cooling to room temperature.

The crystal is in suspension in liquid. Phase contrast, X1000.

are slightly rotated relative to one another about the central axis of the crystal. This feature is not uncommon in multilayered polymer crystals $[3,15]$. In addition, slight pleating may be observed (unbarbed arrows) in the interior of crystal (A) which suggests that the crystal was not perfectly planar as grown. In contrast with this latter type of crystal, the central portions of the multilayered crystals (B) and (C) shown in figure 13 and that shown in figure 14 exhibit a highly complex and confused appearance as a consequence of substantial distortions and/or folding which the constituent lamellae (or more accurately some of the lamellae) in these crystals undergo when the crystals collapse upon drying on a flat substrate. ${ }^{11}$ These crystals may be identified with the more complex "axialitic" species of crystals which are observed under the optical microscope. Such a crystal in suspension in liquid is shown in figure 15 in which the thin "branches" which extend radially outwards from the center of the object shown therein are lamellae (or stacks of lamellae) which are viewed edge-on or nearly so. Such crystals would be expected to undergo substantial deformations when they collapse upon drying on a substrate.

The nearly planar overall character of the simpler multilayered crystals which exhibit no appreciable splaying between their constituent lamellar layers is consistent with the nearly planar nature of the monolayered crystals (Section 3.2 (iii)) grown in the same preparations. The causes of the wide and variable divergences in orientation (i.e., splaying) among the constituent lamellae in the multilayered "axialitic" crystals we have observed has not been pursued in the present study and remains undetermined. Finally, it should be pointed out that essentially planar as well as "axialitic" multilayered crystals such as those shown above in figures 12-15 were also observed in preparations which had not been subjected to filtration and washing prior to being cooled from $T_{c}=140{ }^{\circ} \mathrm{C}$ to room temperature.

\subsection{Crystals Formed in Preparations Cooled to $T_{c}=120^{\circ} \mathrm{C}$}

The onset of cloudiness and hence crystal growth in these preparations occurred before temperature equilibrium at $T_{c}=120^{\circ} \mathrm{C}$ was attained in the solutions following transfer from the $T_{s}$ bath to the $T_{c}$ bath. Both six-sectored monolayered crystals as well as multilayered crystals whose constituent terraces were sixsectored were formed in individual preparations. An examination of these crystals in suspension in liquid revealed that, as was the case for $T_{c}=140{ }^{\circ} \mathrm{C}$, the monolayered crystals formed in the $T_{c}=120^{\circ} \mathrm{C}$ preparations (both filtered and unfiltered) were essentially planar, i.e., they exhibited an apparently linear profile when viewed edge-on as shown in figure 16. Furthermore, multilayered crystals similar to the different ones (i.e., the nearly planar and the "axialitic" ones) which were observed in the $T_{c}=140{ }^{\circ} \mathrm{C}$ preparations were seen under the optical microscope in the $T_{c}=$ $120{ }^{\circ} \mathrm{C}$ preparations. The only apparent difference in

\footnotetext{
11 The electron micrograph in figure 13 was recorded after prolonged exposure of the crystals to the electron beam. Although the polymer in the outer portions of the crystals had volatilized, and although most of the polymer in the interior of crystal (A) had also volatilized, this was not the case in the central region of crystals (B) and (C) as evidenced by the opacity of the inner portions of these latter crystals which opacity remained indefinitely. Bassett [5] has previously observed that although chain scission resulting in the volatilization of the polymer is the predomina thicker regions in POM crystals did not volatilize indicating the incidence of cross-linking.
} 


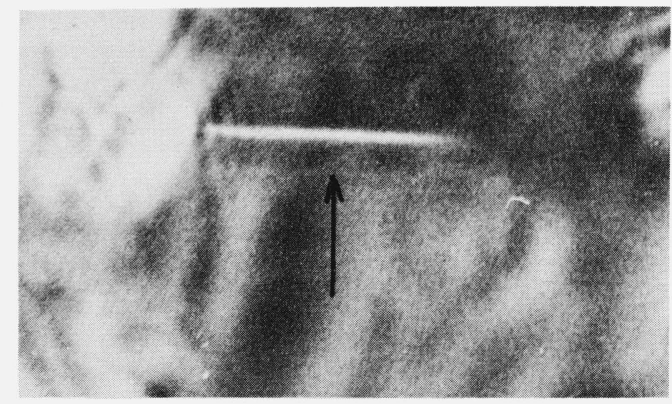

FIGURE 16. Optical micrograph showing an edge-on view of a monolayered crystal (arrow) formed in a $\mathrm{T}_{\mathrm{c}}=120^{\circ} \mathrm{C}$ preparation which had not been filtered and washed prior to cooling to room temperature.

The crystal is in suspension in liquid. Phase contrast, X1200.

habit between the crystals formed in the $T_{c}=120^{\circ} \mathrm{C}$ and those formed in the $T_{c}=140{ }^{\circ} \mathrm{C}$ preparations was the fact (which was only detectable in the electron microscope, see below) that the $\{10 \overline{1} 0\}$ lateral edges of the monolayered crystals as well as the lateral edges of the basal lamella in the multilayered crystals formed in the $T_{c}=120{ }^{\circ} \mathrm{C}$ preparations were usually faintly cusped, i.e., slightly concave relative to the interior of the lamellae. This feature which was observed in crystals from both filtered and unfiltered preparations is illustrated and discussed below.

The monolayered crystal shown in figure 17 was formed in a preparation which had not been filtered prior to the final cooling of the preparation to room temperature from $120{ }^{\circ} \mathrm{C}$. The multilayered crystal shown in figure 18 was formed in a preparation which had been subjected to filtration and washing prior to being allowed to cool to room temperature. Although slight, the cusped character of the edges of the lamella shown in figure 17, the edges of the basal layer of the crystal shown in figure 18 and the visible edges shown in figure 19 of lamellae $(\mathbf{E})$ and $(\mathbf{F})$ from a filtered preparation, is nonetheless evident.

One would expect on the basis of the previous studies which were summarized and discussed in Section 1 (with reference to fig. 1) that at a sufficiently high undercooling SS type lamellae such as those shown in figures 16-18 would be formed rather than hexagonal ones. The manifestation of the incipient SS lateral profile illustrated above in lamellae from both the filtered as well as the unfiltered preparations indicates that this feature is a characteristic of the crystals as they grew in the $T_{c}=120^{\circ} \mathrm{C}$ bath, and this is confirmed by the ensuing observations. Thus, in addition to eliminating uncrystallized polymer from the preparation, the application of the filtration and washing procedure at $120^{\circ} \mathrm{C}$ resulted in a very mild etching of the lamellae, which etching was similar in character to that observed in the lamellae from filtered $T_{c}=140{ }^{\circ} \mathrm{C}$ preparations, as illustrated in the "replica" shown in figure 20 in which $(\mathrm{G})$ and $(\mathrm{H})$ are portions of two different partially overlapping lamellae (same preparation as crystals shown in figs. 18 and 19). The portions
(JK) and (LM) of the respective edges of these lamellae intersect one another almost at right angles at $(\mathrm{N})$. Narrow voids which are oriented with their longer dimension preferentially parallel to the edge $(\mathrm{JK})$ in crystal $(\mathrm{G})$ and to the edge $(\mathrm{LM})$ in crystal $(\mathrm{H})$ may be

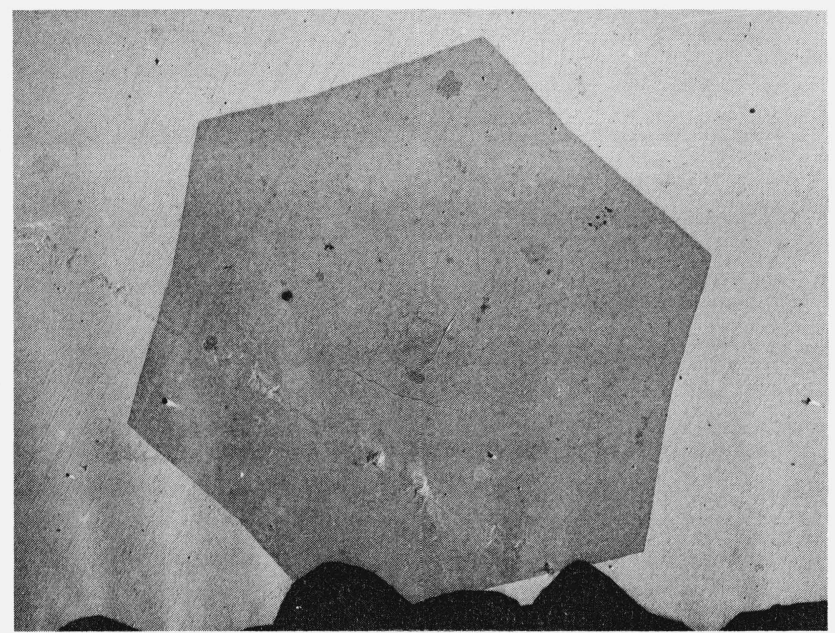

FIGURE 17. Electron micrograph of a monolayered crystal formed in $a \mathrm{~T}_{\mathrm{c}}=120{ }^{\circ} \mathrm{C}$ preparation which had not been filtered and washed prior to cooling to room temperature.

$\mathrm{P}_{\mathrm{t}} / \mathrm{Pd}$ shadowed, $\mathrm{X} 2800$.

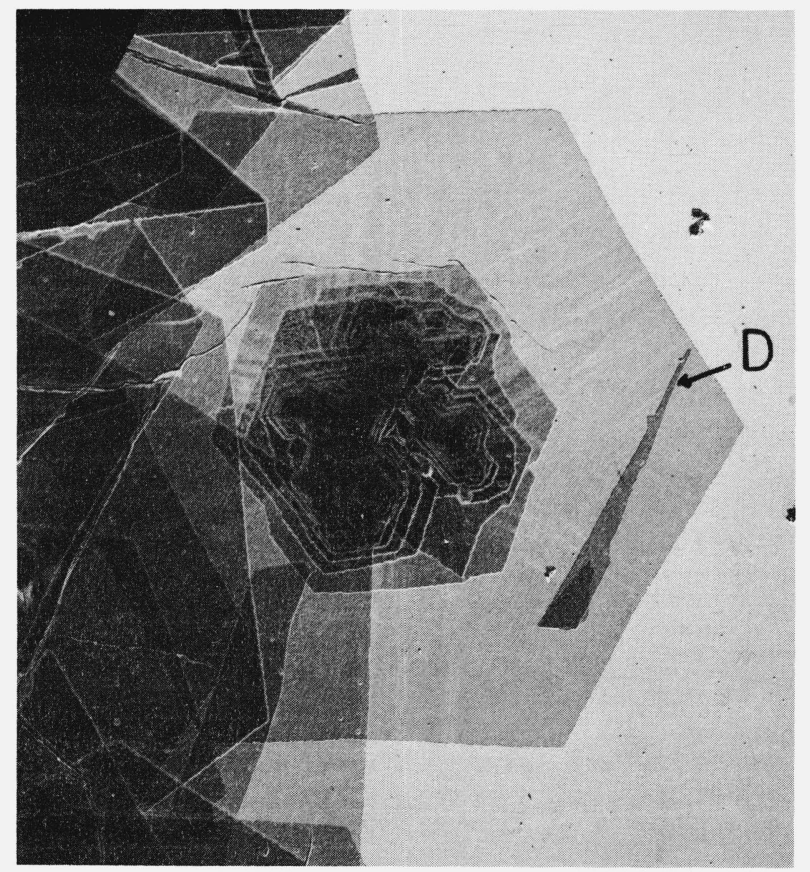

FIGURE 18. Electron micrograph of a multilayered crystal formed in $a \mathrm{~T}_{\mathrm{c}}=120{ }^{\circ} \mathrm{C}$ preparation which had been filtered and washed prior to cooling to room temperature.

(D) is a fragment which had broken off another crystal. $\mathrm{Pt} / \mathrm{Pd}$ shadowed, $\mathrm{X} 3700$. 
seen in figure 20 (e.g., see arrows). In addition to this asymmetric etching which follows the orientation of the $\{10 \overline{10}\}_{f}$ planes along which the chain molecules are folded in the constituent sectors of these six-sectored lamellae, as was the case in the lamellae in the $T_{c}=$

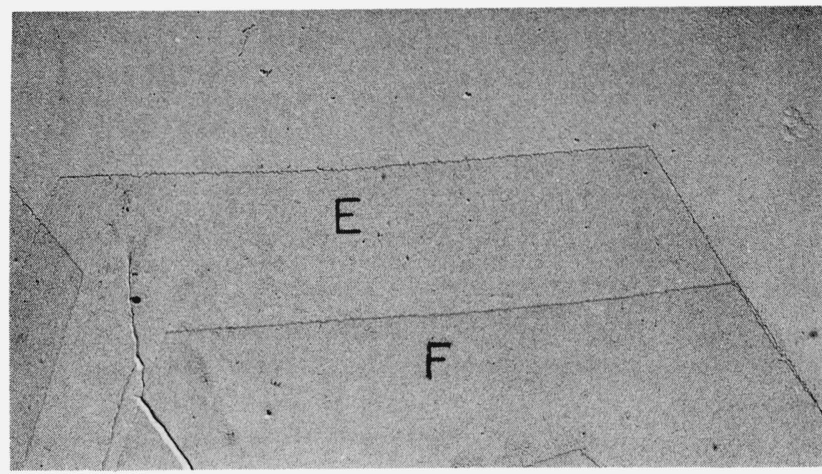

FiguRE 19. Electron micrograph ('replica') showing edges of two overlapping lamellar crystals $(E)$ and $(F)$ formed in a $\mathrm{T}_{\mathrm{c}}=120^{\circ} \mathrm{C}$ preparation which had been filtered and washed at $120^{\circ} \mathrm{C}$ prior to cooling to room temperature.

$\mathrm{Pt} / \mathrm{Pd}$ shadowed, $\mathrm{X} 5800$.
$140{ }^{\circ} \mathrm{C}$ crystals which had been filtered and washed, the nibbled appearance of the edges (JK) and (LM) indicates that some etching has also occurred at the periphery of the lamellae. This latter feature coupled with the incidence of etching in the interior of the lamellae eliminates the possibility that the slightly hexacuspid lateral profile exhibited by the lamellae in the filtered preparation resulted from further growth below $120^{\circ} \mathrm{C}$ due to a failure to eliminate uncrystallized polymer prior to cooling to room temperature. It may also be noted that in the case of the unfiltered preparations, a very narrow thinner outer strip was observed bordering the entire periphery of the lamellae. The incidence of this feature, which escapes detection at low magnifications (e.g., fig. 17) in the electron microscope is illustrated in the high magnification "replica" shown in figure 2l. While the occurrence of the very narrow thinner outer strip around the entire periphery of the lamellae from the unfiltered preparation clearly indicates that all the polymer had not crystallized even after the preparation had been maintained at $120^{\circ} \mathrm{C}$ for 24 hours, it is evident that the width of the strip (which represents the additional growth which the lamellae underwent when the preparation was allowed to cool to room temperature from $120^{\circ} \mathrm{C}$ ) is minimal, thus further confirming that the hexacuspid character of

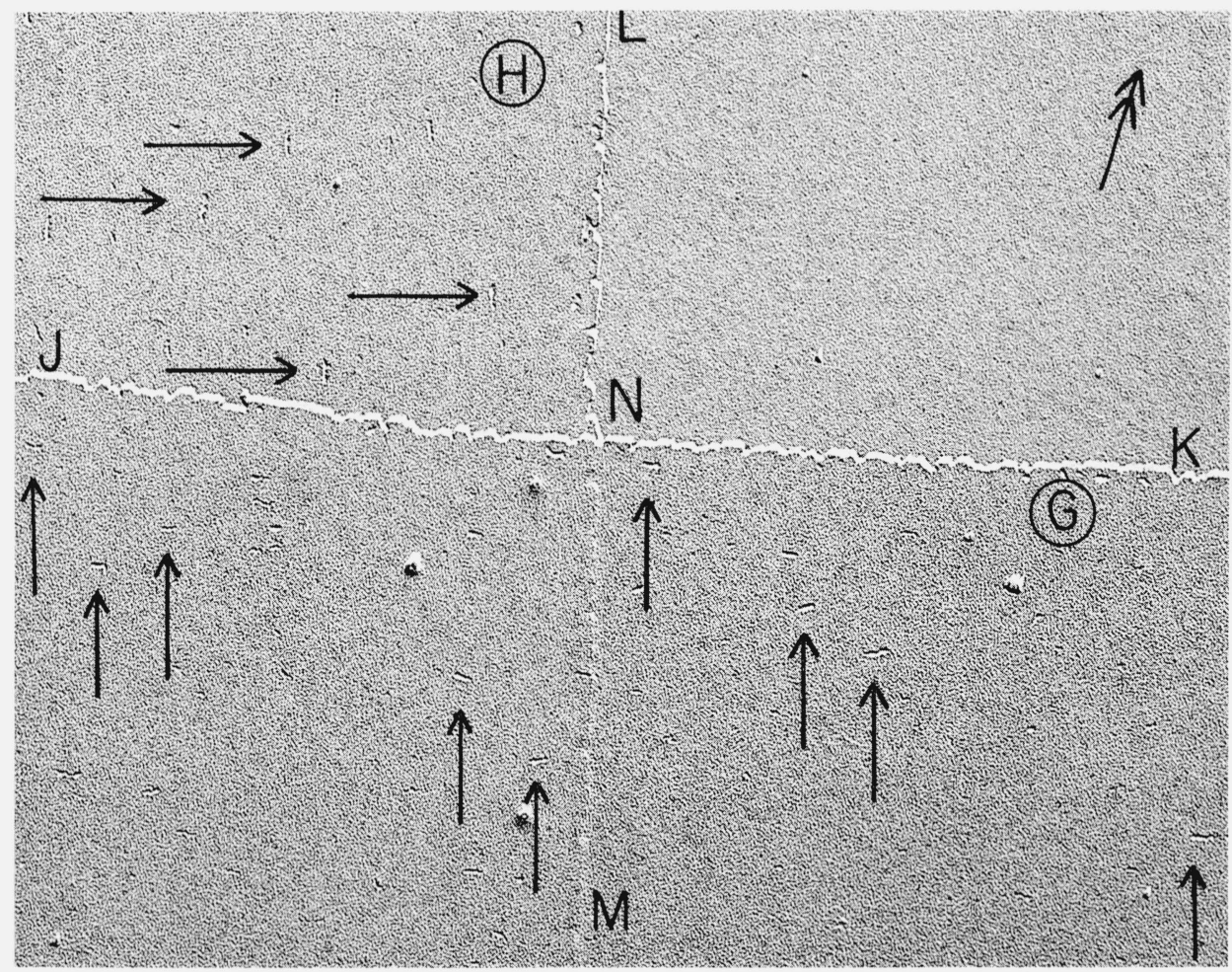

FiguRE 20. Electron micrograph ('replica') showing portions of two partially overlapping lamellar crystals, $(G)$ and $(H)$ formed in a $\mathrm{T}_{\mathrm{c}}=120^{\circ} \mathrm{C}$ preparation which had been filtered and washed at $120{ }^{\circ} \mathrm{C}$ prior to cooling to room temperature.

Lamella $(\mathrm{G})$ lies above lamella $(\mathrm{H})$ in lower left-hand corner of image. Barbed arrow denotes direction of shadowing. Unbarbed arrows point to narrow voids which are oriented with their longer dimension preferentially parallel to side (JK) in lamella (G) and to side (LM) in lamella (H). Pt/Pd shadowed, X29000. 


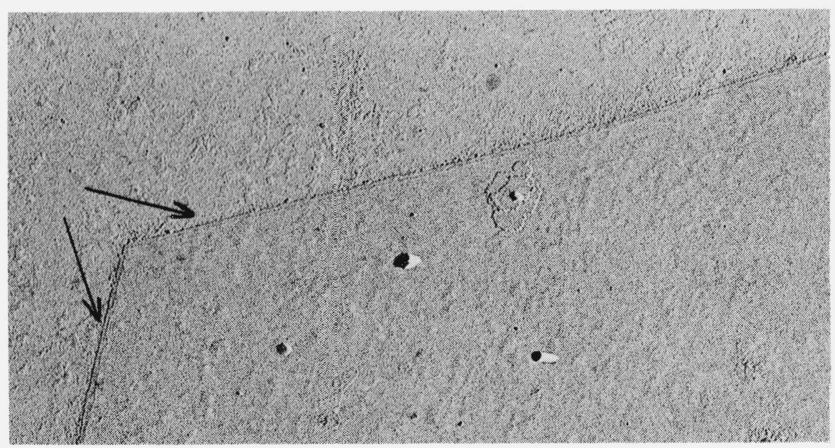

FIGURE 21. Electron micrograph ('replica') showing a portion of the periphery of a monolayered crystal formed in a $\mathrm{T}_{\mathrm{c}}=120{ }^{\circ} \mathrm{C}$ preparation which had not been filtered and washed at $120{ }^{\circ} \mathrm{C}$ prior to cooling to room temperature.

Arrows point to a thinner narrow strip which borders the periphery of the lamella and which was formed when the preparation cooled from $120{ }^{\circ} \mathrm{C}$ to room temperature. $\mathrm{Pt} / \mathrm{Pd}$ shadowed, X12000.

the lateral profile of the sedimented lamellae, which feature is discernable at low magnifications under the electron microscope (e.g., fig. 17), is not merely a consequence of any additional growth which occurred below $120^{\circ} \mathrm{C}$ due to the omission of the filtration procedure.

As was the case for the monolayered crystals formed in the $T_{c}=140{ }^{\circ} \mathrm{C}$ preparations, the essentially linear profile exhibited by the incipient SS monolayered crystals formed in the $T_{c}=120{ }^{\circ} \mathrm{C}$ preparations when the crystals are viewed edge-on in suspension in liquid argues against the incidence of regular fold staggering in these crystals. These crystals are, however, not perfectly planar, as evidenced by the incidence of pleats in the central region of the lamellae. A view at high magnification of a pleat (barbed arrow) near the center of a monolayered crystal is shown in figure 22 , in which the unbarbed arrows point in the direction of faint streaks (due to diffraction contrast) which are of the same kind as those seen in the $T_{c}=140^{\circ} \mathrm{C}$ crystals. Such streaks may also be seen (running characteristically at right angles to the edges of the lamellae) in the basal lamella of the multilayered crystal in figure 18. These streaks also attest to the not perfectly planar character of the monolayered SS type lamellae as well as the constituent lamellae in the nonaxialitic multilayered crystals formed in the $120^{\circ} \mathrm{C}$ preparations. The departure from planarity in these crystals is however apparently slight as was the case in the monolayered crystals and the nonaxialitic multilayered crystals formed in the $T_{c}=140{ }^{\circ} \mathrm{C}$ preparations, and may be attributed to the same causes (i.e., those indicated in Section 3.2 (iii), with reference to figs. 8-10).

\subsection{Crystals Formed in Solutions Cooled to $T_{c}=114^{\circ} \mathrm{C}$, $T_{c}=100{ }^{\circ} \mathrm{C}$ and $T_{c}=80^{\circ} \mathrm{C}$}

(i) General Remarks

As was the case for the $T_{c}=120^{\circ} \mathrm{C}$ preparations, the onset of crystallization occurred before temperature

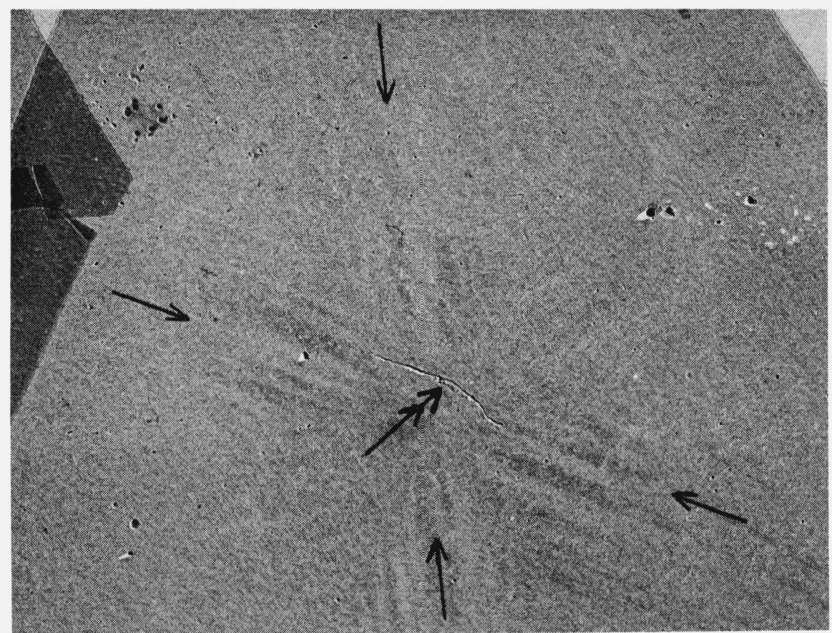

FIGURE 22. Electron micrograph of the central portion of a monolayered crystal formed in a $\mathrm{T}_{\mathrm{c}}=120^{\circ} \mathrm{C}$ preparation which had not been filtered and washed at $120^{\circ} \mathrm{C}$ prior to cooling to room temperature.

Barbed arrow points to a pleat near the center of the crystal. Unbarbed arrows point in the direction of streaks due to diffraction contrast. $\mathrm{Pt} / \mathrm{Pd}$ shadowed, $\mathrm{X} 5300$.

equilibrium was attained in the polymer solutions after they were transferred to the baths at $T_{c}=114{ }^{\circ} \mathrm{C}$, $T_{c}=100{ }^{\circ} \mathrm{C}$ or $T_{c}=80{ }^{\circ} \mathrm{C}$. It could be readily predicted on the basis of the observations made on the crystals formed in the unfiltered $T_{c}=140{ }^{\circ} \mathrm{C}$ and $T_{c}=120{ }^{\circ} \mathrm{C}$ preparations that the amount of residual polymer remaining in solution in the $T_{c}=114{ }^{\circ} \mathrm{C}, 100^{\circ} \mathrm{C}$ or 80 ${ }^{\circ} \mathrm{C}$ preparations would be negligible. Crystals formed in preparations which had been subjected to filtration at $T_{c}$ as well as preparations in which this aftertreatment procedure was omitted were examined. In the case of the unfiltered $T_{c}=114{ }^{\circ} \mathrm{C}$ preparations further growth upon cooling to room temperature was negligible. No detectable evidence of further growth upon final cooling from $T_{c}$ to room temperature was observed in crystals formed in the unfiltered $T_{c}=100$ ${ }^{\circ} \mathrm{C}$ or the $T_{c}=80{ }^{\circ} \mathrm{C}$ preparations. Furthermore, no detectable evidence of any etching was observed in any of the filtered preparations. In short, the characteristics of the habits of the crystals formed in the $T_{c}=114{ }^{\circ} \mathrm{C}, T_{c}=100{ }^{\circ} \mathrm{C}$ and the $T_{c}=80^{\circ} \mathrm{C}$ preparations which are described below are representative of the manner in which the crystals grew in the crystallization baths maintained at $T_{c}$.

\section{(ii) Crystals Formed in Solutions Cooled to $T_{c}=114{ }^{\circ} \mathrm{C}$}

The crystals formed in the $T_{c}=114{ }^{\circ} \mathrm{C}$ preparations exhibited a distinctly dendritic mode of lateral propagation, i.e., their lateral growth habit was akin to the SD habit depicted schematically in figure ld. An example of the crystals formed in the $T_{c}=114{ }^{\circ} \mathrm{C}$ preparations is shown in figure 23 in which the following characteristic features exhibited by these crystals may be noted. The first feature is the overall profile of the basal lamella which is pronouncedly star-like in shape; 


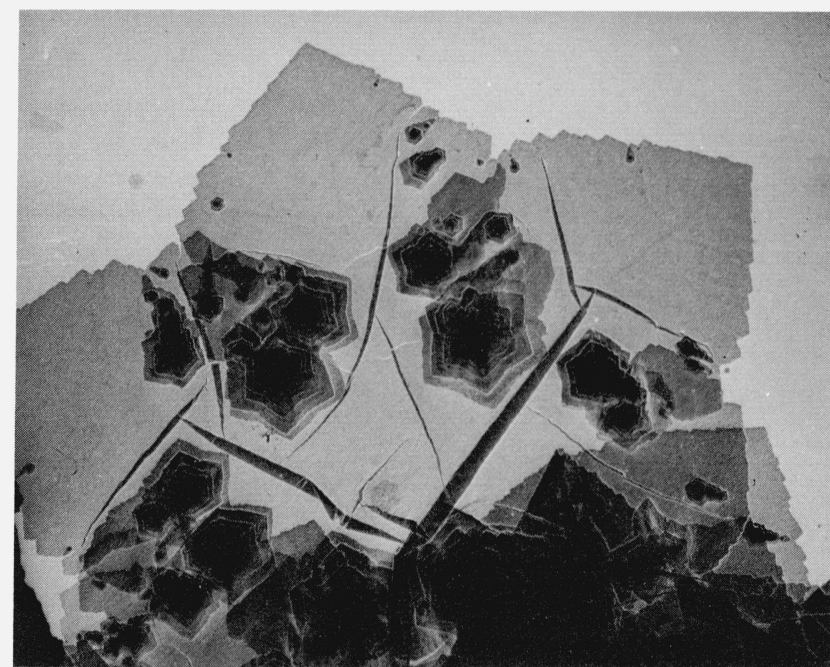

FiguRE 23. Electron micrograph of a dendritic crystal formed in $a \mathrm{~T}_{\mathrm{c}}=114{ }^{\circ} \mathrm{C}$ preparation.

Note the microfaceted character of the edges of the basal lamella, and the pleats in the interior of the crystal. $\mathrm{Pt} / \mathrm{Pd}$ shadowed, $\mathrm{X} 3100$.

this feature contrasts with the hexagonal profile and the incipient hexacuspid profile exhibited by the lamellae we have described in sections 3.2 and 3.3 , respectively. The second feature to be noted is the distinctly microfaceted nature of the periphery of the basal lamella. This feature contrasts with the smooth (i.e., no microfaceting observed) lateral edges of the hexagonal and incipiently hexacuspid lamellae formed in the $T_{c}=140{ }^{\circ} \mathrm{C}$ and the $T_{c}=120^{\circ} \mathrm{C}$ preparations, respectively. The third characteristic is the intermittent incidence of the generation of screw-dislocation-like defects at the periphery of the basal lamella during its outward growth, from which defects additional chain folded terraces develop in spiral-like fashion upon the basal lamella. It may be noted that the generation of these defects, and hence it may be adduced the onset of (SD) lateral growth, occurred after the basal lamella had attained between one third to one half its ultimate maximum outward span. Fourth, numerous pleats which may be readily seen in figure 23 were always observed in the interior of these crystals. The occurrence of these pleats in the crystals as seen lying on a flat substrate suggests in itself that the overall conformation of these cyrstals as-grown is not planar. Indeed, as described below, they exhibit a distinguishably non-planar conformation when viewed in suspension in liquid under the optical microscope.

Two different views of a crystal in suspension in liquid as seen under the optical microscope are shown in figure 24 . In figure 24 a the crystal is oriented with its central axis of six-fold symmetry slightly inclined with respect to the normal to the field of view. The bright "spots" which may be seen in the interior of the crystal correspond to spiral terraces such as those which may be clearly seen in figure 23 . Figure $24 \mathrm{~b}$ depicts the same crystal as seen edge-on, i.e., with its central axis in the plane of the field of view. The arrow in
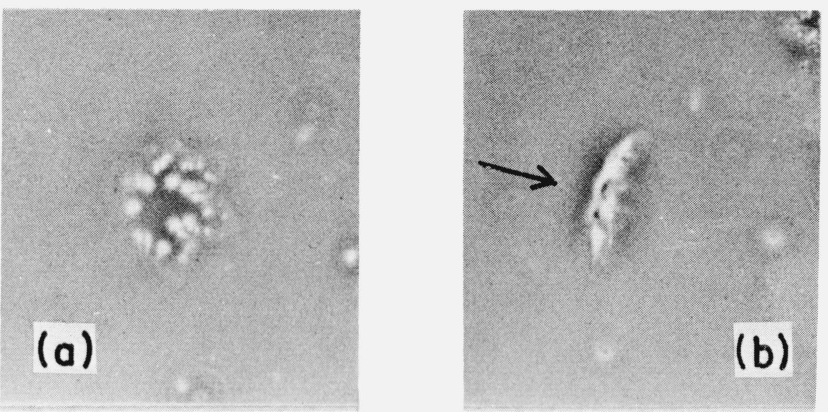

FIGURE 24. Optical micrographs showing two perspectives of a dendritic crystal formed in a $\mathrm{T}_{\mathrm{c}}=114^{\circ} \mathrm{C}$ preparation.

The crystal is in suspension in liquid. (a) Central axis of crystal is slightly inclined with respect to the normal to the field of view. (b) The same crystal oriented as seen edge on. i.e., with its central axis (arrow) in the plane of the field of view. Phase contrast, X500.

figure $24 \mathrm{~b}$ which points to the pole of the crystal also corresponds in direction to that of the central axis of the crystal. The crystal exhibits an overall appreciably curved profile in this latter perspective suggesting that these crystals possess a dished overall conformation. Their departure from planarity, although still slight in comparison with crystals formed in the $T_{c}=100{ }^{\circ} \mathrm{C}$ and $T_{c}=80{ }^{\circ} \mathrm{C}$ preparations (see later), is more pronounced than that of the monolayered and non-axialitic multilayered crystals formed in the $T_{c}=140{ }^{\circ} \mathrm{C}$ and $T_{c}=$ $120^{\circ} \mathrm{C}$ preparations. It may be roughly estimated from their edge-on profile as seen in suspension in liquid that the overall radius of curvature of the crystals such as those shown in figures 23 and 24 is about $20-30 \mu \mathrm{m}$.

\section{(iii) Crystals Formed in Solutions Cooled to $\boldsymbol{T}_{c}=100{ }^{\circ} \mathrm{C}$ and $\boldsymbol{T}_{c}=80^{\circ} \mathrm{C}$}

In addition to the fact that the onset of crystallization occurred well before temperature equilibrium was attained at $T_{c}$, the crystallization of the polymer in these preparations was also characterized by the fact that the inception of new crystals occurred over a wide temperature range as the solutions cooled to $T_{c}$. The populations of crystals formed in the $T_{c}=100{ }^{\circ} \mathrm{C}$ and the $T_{c}=80{ }^{\circ} \mathrm{C}$ preparations were very similar to one another in that: (a) they consisted in each case of a mixture of differently curved six-fold symmetrically shaped dendritic chain folded crystals whose lateral dimensions (as measured from pole to periphery) and corresponding conformations (curvatures) varied widely from crystal to crystal over essentially the same range for both $T_{c}=100{ }^{\circ} \mathrm{C}$ and $T_{c}=80{ }^{\circ} \mathrm{C}$. and that (b) the lower the temperature at which individual crystals started to grow during the cooling of the solutions to $T_{c}$, as evidenced inter alia (see below) by their relatively smaller lateral dimensions, the more pronouncedly curved was their overall conformation.

The phase contrast optical micrographs shown in figures 25 to 27 represent crystals (in suspension in liquid and randomly oriented with respect to the viewing direction) grown in a $T_{c}=100{ }^{\circ} \mathrm{C}$ preparation, those shown in figures 28 and 29 represent crystals grown in a $\mathrm{T}_{c}=80{ }^{\circ} \mathrm{C}$ preparation. Crystal (N) in 


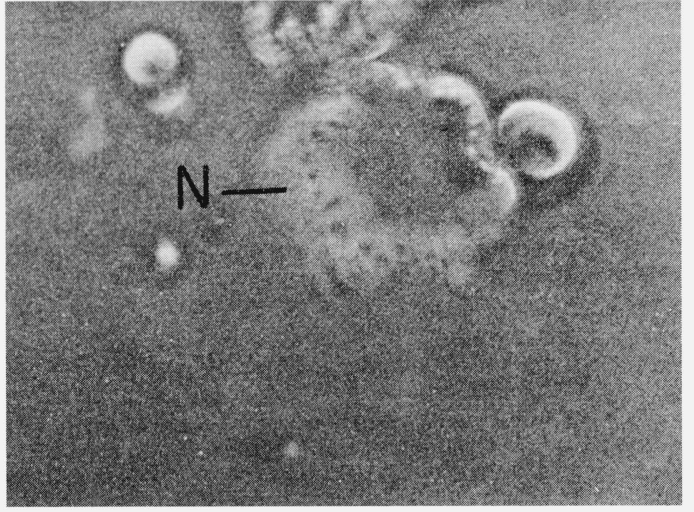

FIGURE 25. Optical micrograph of crystals formed in a $\mathrm{T}_{\mathrm{c}}=100{ }^{\circ} \mathrm{C}$ preparation.

Crystals are in suspension in liquid. Crystal (N) which is similar to crystal (Q) in figure 28 [12] is representative of the largest and least curved (referring to the overall conformation of the crystal) crystals formed in the $T_{c}=100{ }^{\circ} \mathrm{C}$ preparations. Phase contrast, $\mathrm{X} 825$.

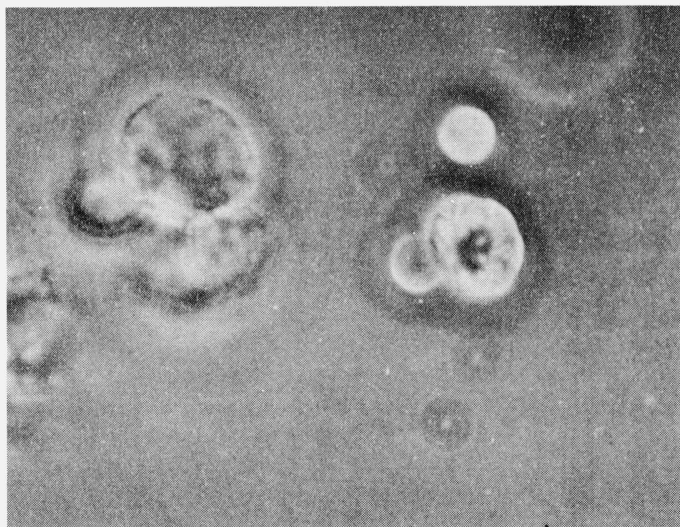

FiguRE 26. Optical micrograph of crystals formed in a $\mathrm{T}_{\mathrm{c}}=100$ ${ }^{\circ} \mathrm{C}$ preparation.

Crystals are in suspension in liquid, see text. Phase contrast, X825.

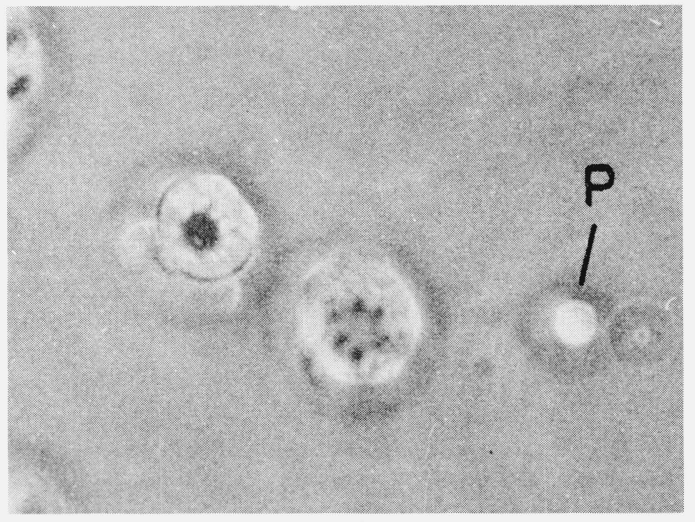

Figure 27. Optical micrograph of crystals formed in a $\mathrm{T}_{\mathrm{c}}=100$ ${ }^{\circ} \mathrm{C}$ preparation.

Crystals are in suspension in liquid, see text. Phase contrast, X825.

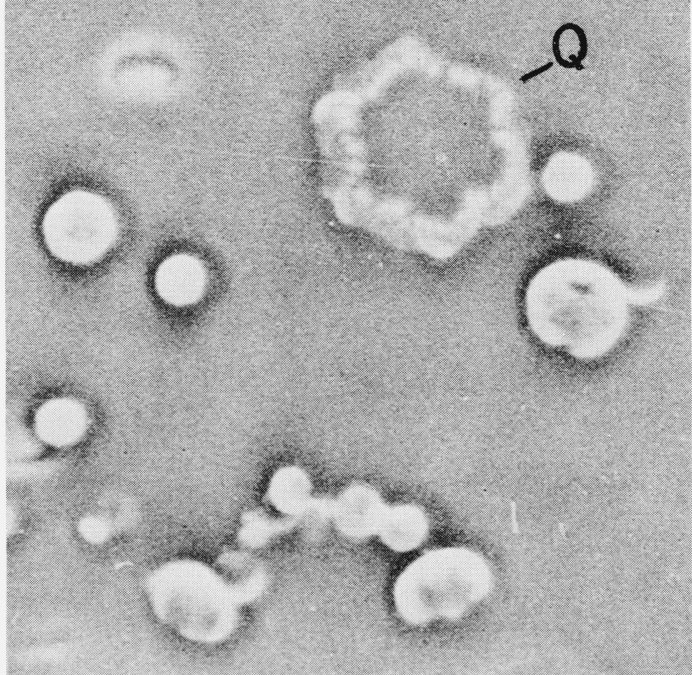

Figure 28. Optical micrograph of crystals formed in a $\mathrm{T}_{\mathrm{c}}=80^{\circ} \mathrm{C}$ preparation.

Crystals are in suspension in liquid, see text. Phase contrast, X825.

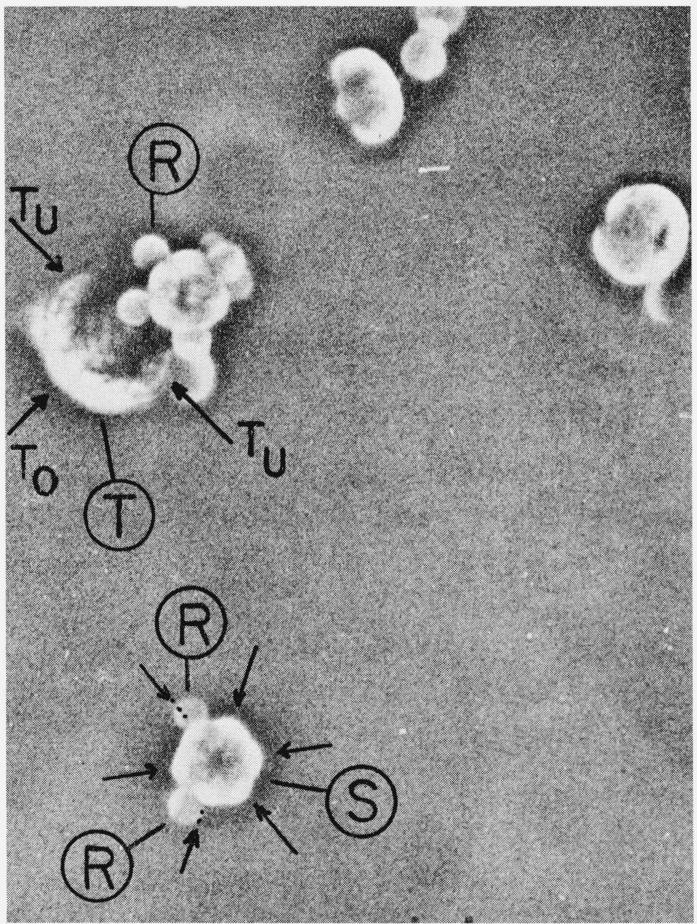

Figure 29. Optical micrograph of crystals formed in a $\mathrm{T}_{\mathrm{c}}=80^{\circ} \mathrm{C}$ preparation.

Crystals are in suspension in liquid. see text. Phase contrast, X825.

figure $25\left(T_{c}=100{ }^{\circ} \mathrm{C}\right)$ and crystal $(\mathrm{Q})$ in figure 28 $\left(T_{c}=80{ }^{\circ} \mathrm{C}\right)$, which are similar to one another, ${ }^{12}$ are representative of the largest and least curved (i.e., their overall conformation is least curved) among the

${ }^{12}$ Whereas crystal $(Q)$ in figure 28 is oriented with its central axis very nearly normal to the field of view, the central axis of crystal $(\mathrm{N})$ in figure 25 is distinctly inclined with respect to the viewing direction. 
dendritic crystals formed in the $T_{c}=100{ }^{\circ} \mathrm{C}$ and the $T_{c}=80^{\circ} \mathrm{C}$ preparations respectively, whereas crystal $(\mathrm{P})$ in figure $27\left(T_{c}=100{ }^{\circ} \mathrm{C}\right)$ and crystals $(\mathrm{R})$ in figure $29\left(T_{c}=80{ }^{\circ} \mathrm{C}\right)$ which are hollow bowl-shaped (see below) are representative of the smallest and most pronouncedly curved crystals formed in these preparations. Crystals whose sizes and corresponding overall curvatures fall between the examples identified above of the opposite extremes of the spectrum of differently curved crystals formed in these preparations may also be seen in the fields of view shown in figures 25 to 29 .

With the qualified exception of the largest and least curved among the crystals formed in each preparation, i.e., crystals such as (N) in figure 25 and $(\mathrm{Q})$ in figure 28 whose growth spanned the entire temperature range over which crystallization occurred in these preparations and whose overall conformation will be considered later in this section, the remaining more pronouncedly curved crystals formed in the $T_{c}=100^{\circ} \mathrm{C}$ and the $T_{c}=80^{\circ} \mathrm{C}$ preparations were hollow bowl-like in shape. The diameter of the smallest and most curved among these bowls was $4-5 \mu \mathrm{m}$ (e.g. crystals R. fig. 29). The overall conformations of these bowls of different sizes were six-fold symmetrical in character as shown schematically in figure 30. Figure 30a represents a bowl as viewed at right angles to its

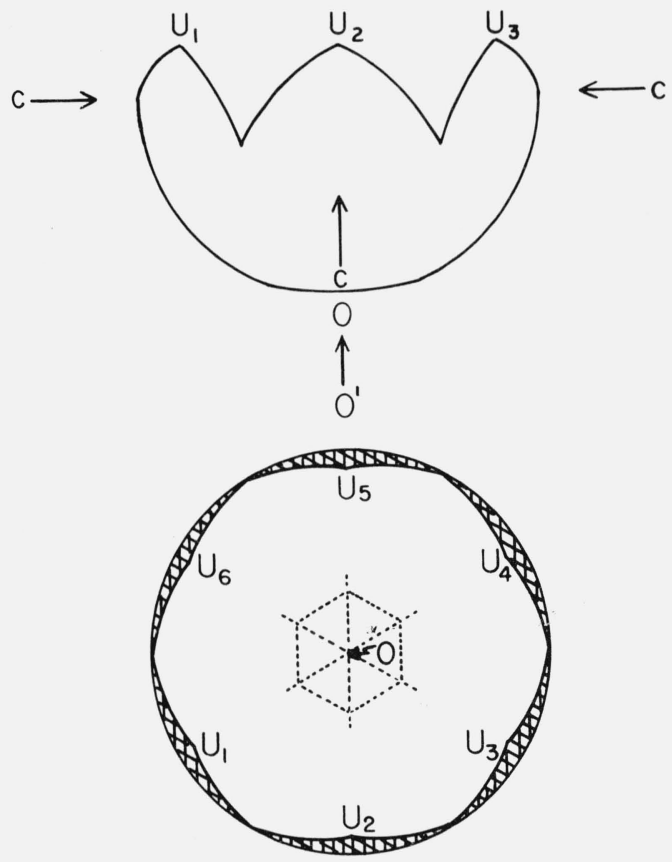

(a)

(b)

FIGURE 30. Schematic representation of six-fold symmetrical character of hollow bowl-shaped crystals formed in the $\mathrm{T}_{\mathrm{c}}=100$ ${ }^{\circ} \mathrm{C}$ and the $\mathrm{T}_{\mathrm{c}}=80^{\circ} \mathrm{C}$ preparations. (a) Side view along a direction normal to the central axis $\left(\mathrm{O}^{\prime} \mathrm{O}\right)$ of the crystal. (b) A view depicting a bowl as seen along its central axis in relation to a hexagonal six-sectored precursor. The points $(U)$ denote the leading extremities of the bowl in which the orientation of the surface of the crystal and hence the $c$ axis, changes through $\sim 90^{\circ}$ between the pole $(O)$ and the extremities $(U)$. central axis of six-fold symmetry $\mathrm{O}^{\prime} \mathrm{O}(\mathrm{O}$ is the pole of the crystal), and figure 30b represents the bowl as seen along its central axis in relation to a hexagonal sixsectored precursor (see later). As shown in these diagrams the six-fold symmetrical nature of the overall shape of these hollow bowl-shaped objects, which are in fact variously curved 'six-armed' starlike dendrites, is characterized by six longitudes $\left(\mathrm{OU}_{1-6}\right)$ along which the growth of the curved lamellar wall has progressed most rapidly, whereas the development of the wall is effectively slower and tapers off symmetrically between the $(\mathrm{OU})$ longitudes. This six-fold symmetrical variation in the development of the constituent curved lamellar wall in those bowls is evidenced under the optical microscope by a corresponding six-fold symmetrical variation in contrast about the outer circular outline of the bowls (with the exception of the smallest and most pronouncedly curved bowls in which this variation in contrast could not be resolved) when they are viewed along their central axis while in suspension in liquid. This feature may be seen most evidently in the appropriately oriented crystal (S) in figure 29 in which the six regions of higher contrast which are pointed to by unbarbed arrows correspond to the hatched regions shown in figure $30 \mathrm{~b}$ at which regions the projected thickness of the curved lamellar wall in the direction parallel to the central axis of the bowl is effectively greater than elsewhere about its circumference in that projection. As shown schematically in figure 30a the orientation of the surface of the lamellar wall and hence the $c$ axis in all these bowls changes through $\sim 90^{\circ}$ or more on proceeding from the pole $(\mathrm{O})$ to the extremities $\mathrm{U}_{1-6}$. This feature is revealed by examining the crystals at right angles to their central axis as is the case for crystal (T) in figure 29 where the arrows $\left(\mathrm{T}_{\mathrm{o}}\right)$ and $\left(\mathrm{T}_{\mathrm{u}}\right)$ are drawn normal to the outer surface of the bowl at its pole and at or very near its rim, respectively. Additional aspects concerning the shapes of the hollow bowl-like crystals of various sizes whose main conformational characteristics have been outlined above, as well as the matter of the conformation of the largest among the crystals formed in these preparations (i.e., crystals such as $(\mathrm{N})$ in fig. 25 and (Q) in fig. 28) which has not been dwelt on so far, will be considered at the end of this section where they will be discussed in connection with the morphological characteristics which the variously curved crystals exhibited in the electron microscope. These latter characteristics which are exemplified in figures 31 to 38 are described below.

The examples of crystals shown in figures 31 to 37 which have been drawn in part from a $T_{c}=100{ }^{\circ} \mathrm{C}$ preparation (figs. 31-34) and in part from a $T_{c}=80{ }^{\circ} \mathrm{C}$ preparation (figs. $35-37$ ) are illustrative of the spectrum of crystals of different sizes and curvatures which were formed in both the $T_{c}=100{ }^{\circ} \mathrm{C}$ as well as the $T_{c}=80$ ${ }^{\circ} \mathrm{C}$ preparations. It may be noted that the crystals from the $T_{c} 100{ }^{\circ} \mathrm{C}$ preparation are shown in sequence in figures $(31,32)$, figure 33 , and figure 34 according (broadly speaking) to diminishing actual size, as are in essence the crystals from the $80{ }^{\circ} \mathrm{C}$ preparation which 


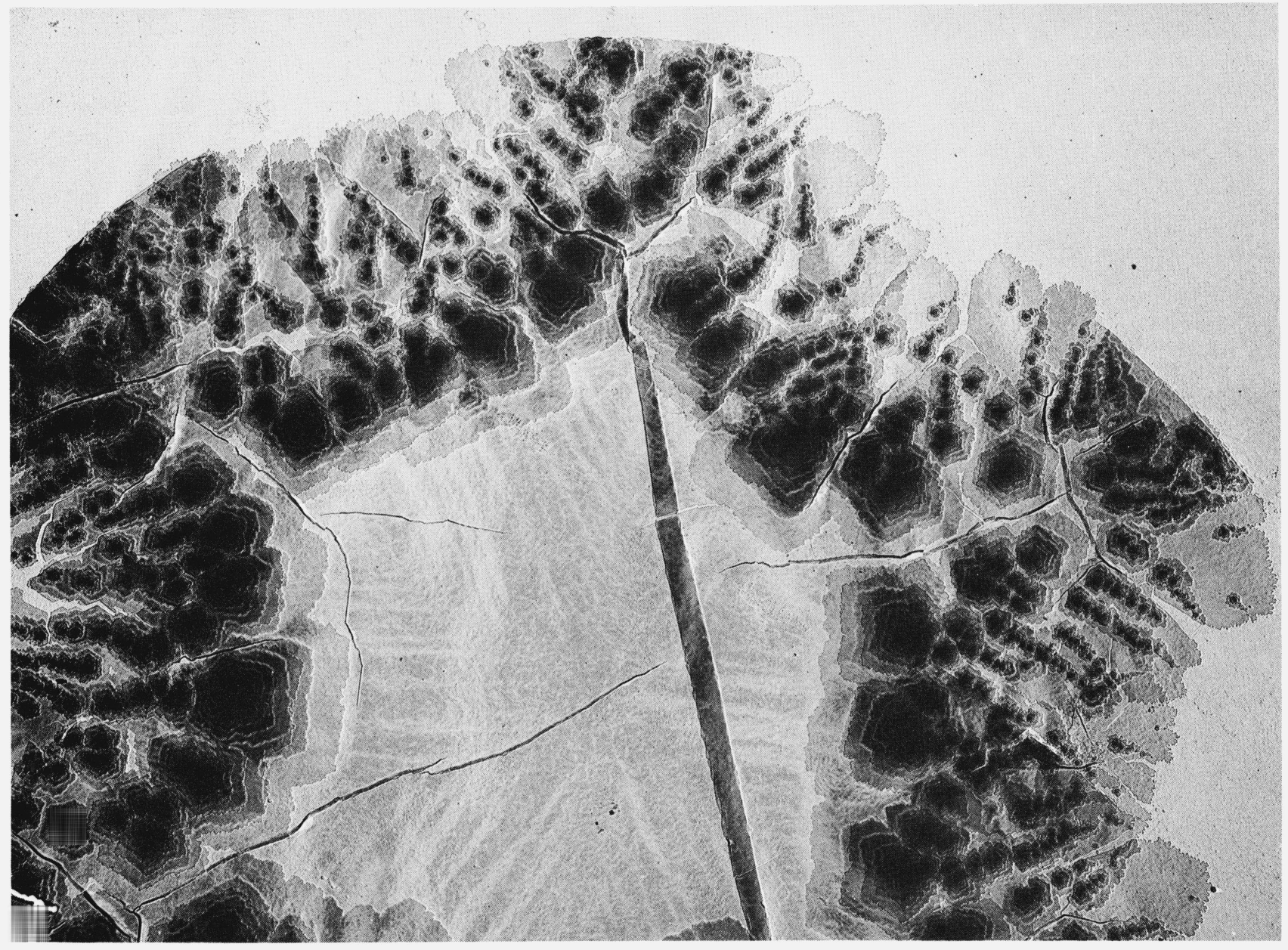

FigURE 31. Electron micrograph of a crystal formed in a $\mathrm{T}_{\mathrm{c}}=100^{\circ} \mathrm{C}$ preparation; see text.

$\mathrm{Pt} / \mathrm{Pd}$ shadowed, $\mathrm{X} 7100$.

are shown in figures 35 to 37 . The crystals shown in figure 31 and figure 32 are illustrative of the larger species of crystals which were formed in the $T_{c}=100$ ${ }^{\circ} \mathrm{C}$ and the $T_{c}=80{ }^{\circ} \mathrm{C}$ preparations, i.e., they correspond quite closely to crystals such as crystal $(\mathrm{N})$ in figure $25\left(T_{c}=100{ }^{\circ} \mathrm{C}\right)$ and crystal $(\mathrm{Q})$ in figure 28 $\left(T_{c}=80^{\circ} \mathrm{C}\right)$. Crystals $(\mathrm{X})$ and $(\mathrm{Y})$ in figure 37 are illustrative of the smaller among the crystals formed in these preparations, i.e., they correspond closely to crystals such as $(\mathrm{P})$ in figure $27\left(T_{c}=100{ }^{\circ} \mathrm{C}\right)$ and $(\mathrm{R})$ in figure $29\left(T_{c}=80^{\circ} \mathrm{C}\right)$. It should be noted that most of the crystals shown in figures 31 to 37 had settled on the carbon film substrate with their central axis normal or preferentially normal to the substrate and subsequently collapsed (were flattened) in that orientation relative to the substrate upon drying, the evident exceptions are: the crystal shown in figure 32 , and the two smaller crystals $(\mathrm{X})$ and $(\mathrm{Y})$ in figure 37 all of which settled and were flattened "sideways" upon the substrate, i.e., with their central axis preferentially parallel to the substrate.

It has been pointed out earlier in this paper that dendritic (multisectored) growth in POM chain-folded crystals is characterized (inter alia) by (a) the recurring generation of screw dislocation-like defects at the periphery of the constituent basal (leading) lamella in such crystals and (b) by the formation through the agency of these defects of additional chain-folded terraces which develop in a spiral-like fashion upon the basal lamella. Since these two features, which 


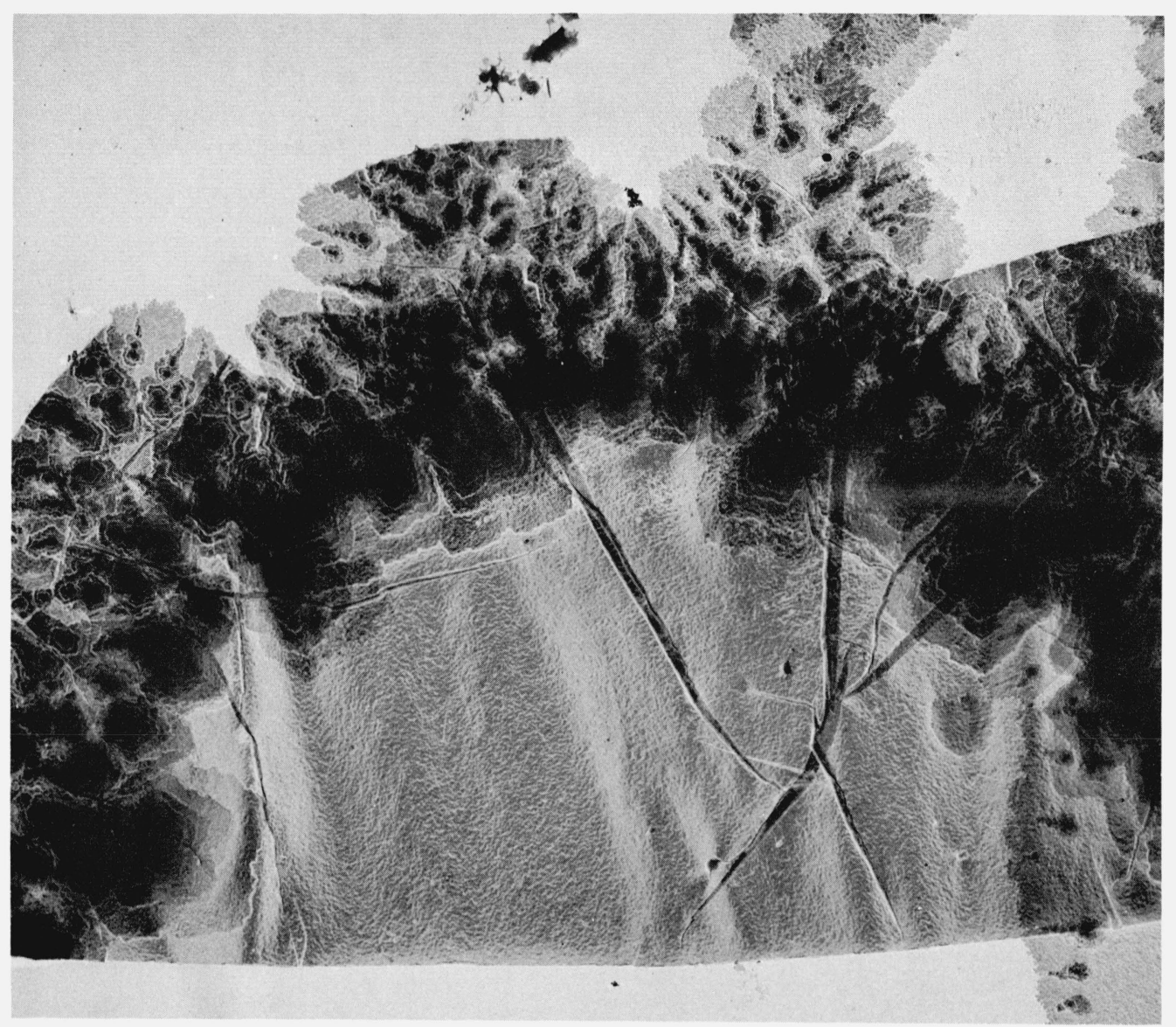

FigURE 32. Electron micrograph of a crystal formed in a $\mathrm{T}_{\mathrm{c}}=100{ }^{\circ} \mathrm{C}$ preparation.

This crystal is similar to that shown in figure 31 , however, it folded about its diameter when it collapsed upon the substrate; see text. $\mathrm{Pt} / \mathrm{Pd}$ shadowed, X8300.

occur in association with the incidence of multisectored lateral growth, distinguish the development of dendrites from that of hexagonal six-sectored and SS type lamellae, it is of interest to note, as may be seen from a consideration of the crystals shown in figures 31 and 32 , that there is a distinct difference in morphology between the central and outer portions of these examples of the largest among the crystals formed in the $T_{c}=100{ }^{\circ} \mathrm{C}$ and the $T_{c}=80{ }^{\circ} \mathrm{C}$ preparations. Whereas the inner (central) portion of the basal lamella in these crystals is devoid of spiral terraces, the development of the outer portions of these crystals is characterized by the profuse formation of spiral terraces. The absence of spiral terrace formation in the inner region of these crystals, coupled with the six-fold symmetrical disposition of the streaks due to diffraction contrast which may be readily seen in the central spiral-terrace-free portion of the basal lamella in the crystal shown in figure 31 , may be readily adduced as evidence that these largest crystals (see also fig. 32) evolved from an initially formed six-sectored precursor lamella, which continued to grow laterally (during the early stages of the cooling of the solution to $T_{c}$ ) in a six-sectored fashion to an appreciable size $(6-7 \mu \mathrm{m}$ as measured from the origin of the basal lamella to the 'row' of spiral terraces closest to the origin), before it underwent a change in lateral habit to multisectored dendritic growth entailing the generation of screw dislocation-like defects and the evolution of spiral terraces therefrom as the crystal continued to grow at 


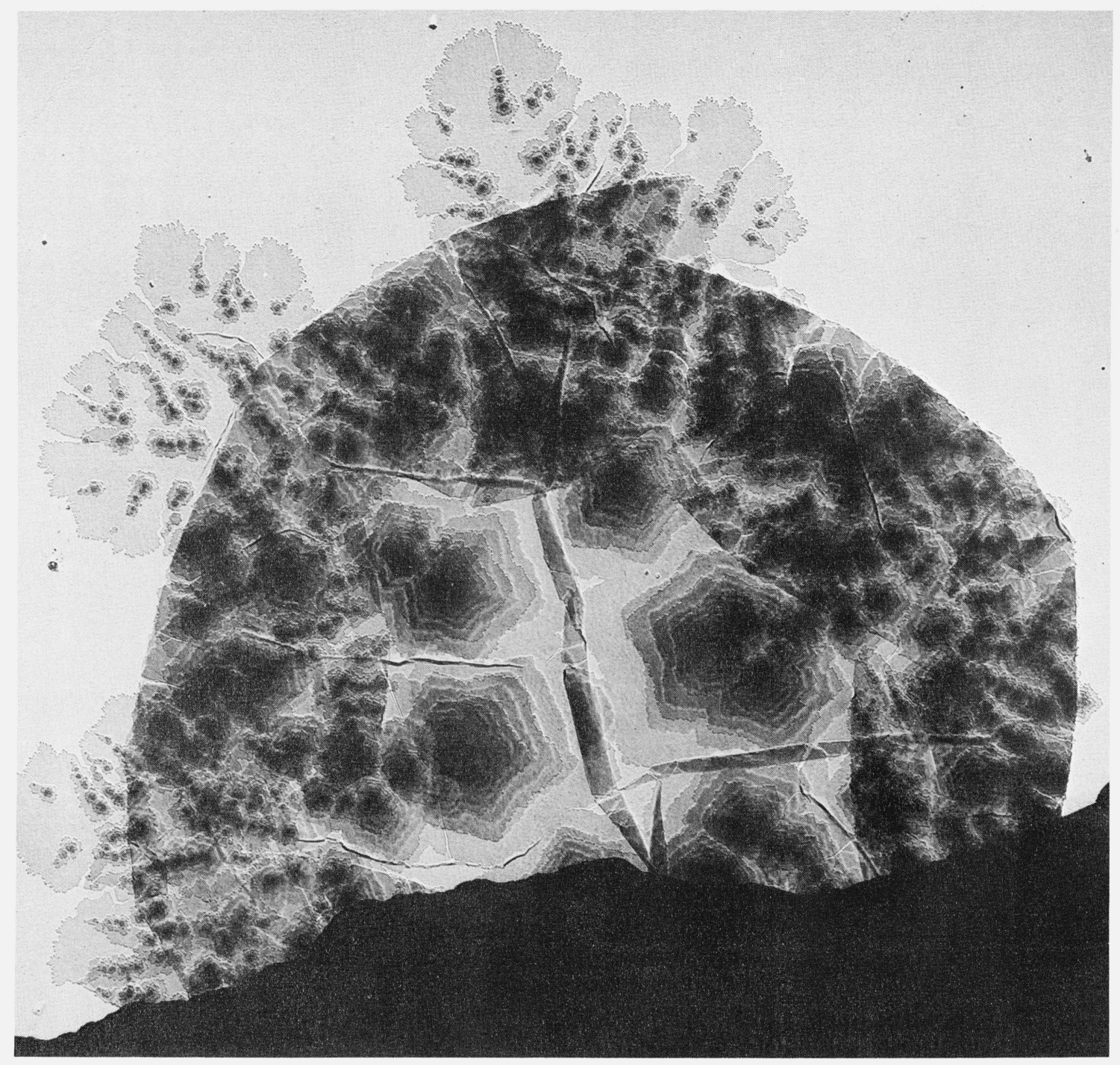

FiguRE 33. Electron micrograph of a crystal formed in a $\mathrm{T}_{\mathrm{c}}=100^{\circ}$ C preparation; see text.

Pt/d shadowed. X8500

lower temperatures. The occurrence of this transformation in lateral habit was not peculiar to these largest crystals whose growth as indicated earlier spanned the entire, or close to the entire temperature range over which crystallization occurred during the cooling of the solutions to $T_{c}$. Thus, as may be seen for example from a consideration of the relatively smaller crystals shown in figures 33 to 35 , their central or polar portion is also free of active screw-dislocation-like defects. However, as may be judged from the entire series of micrographs shown in figures 31 to 37 the lateral extent of the spiral terrace free polar portion of the basal lamella in the crystals formed in both the $T_{c}=100{ }^{\circ} \mathrm{C}$ and the $T_{c}=80{ }^{\circ} \mathrm{C}$ preparations varied from crystal to crystal in a manner commensurate with crystal size and curvature and hence with the order in which the crystals 


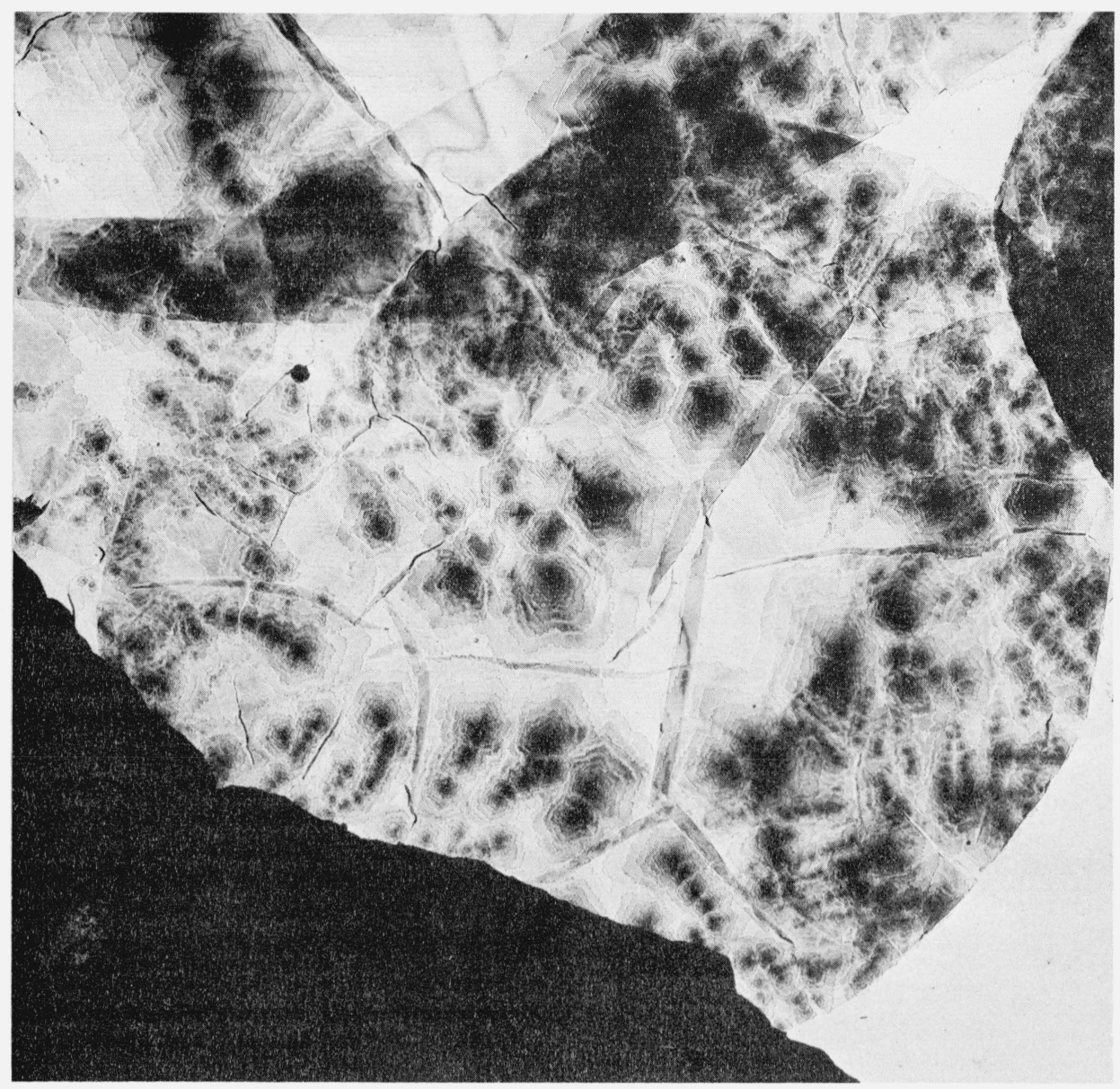

FiguRE 34. Electron micrograph of a crystal formed in a $\mathrm{T}_{\mathrm{c}}=100^{\circ} \mathrm{C}$ preparation; see text.

$\mathrm{Pt} / \mathrm{Pd}$ shadowed, $\mathrm{X} 8300$.

started to grow during the cooling of the solutions to $T_{c}$. Thus, for example, the distance between the center or pole of the basal lamella and the center(s) of the closest set of spiral terraces to it (i.e., the first set of spiral terraces which were initiated at its periphery during growth) is $\sim 6-7 \mu \mathrm{m}$ in the larger among the crystals (figs. 31 and 32 ) as indicated earlier, as compared to $\sim 3 \mu \mathrm{m}$ in the intermediate sized crystals (figs. 33 and 35), and to $\sim 1 \mu \mathrm{m}$ or less in the smaller and more pronouncedly curved crystals such as $(\mathrm{V})$ and $(\mathrm{W})$ in figure 36 and $(\mathrm{X})$ and $(\mathrm{Y})$ in figure 37. This trend clearly illustrates that, not unexpectedly, the onset of dendritic growth involving multisectoring and the generation of screw dislocation-like defects at the periphery of the basal lamella in the crystals formed in the $T_{c}=100{ }^{\circ} \mathrm{C}$ and the $T_{c}=80^{\circ} \mathrm{C}$ preparations manifested itself at an earlier stage in the development of the basal lamella during the course of its growth the lower the temperature at which it started to grow during the cooling of the solutions to $T_{c}$.

In the light of the lateral habits exhibited by the crystals formed in the $T_{c}=120^{\circ} \mathrm{C}$ and the $T_{c}=114{ }^{\circ} \mathrm{C}$ preparations it may be reasonably assumed that the onset of dendritic SD growth during the cooling of the $T_{c}=100{ }^{\circ} \mathrm{C}$ and the $T_{c}=80{ }^{\circ} \mathrm{C}$ preparations to $T_{c}$ occurred most probably at a temperature in the range 


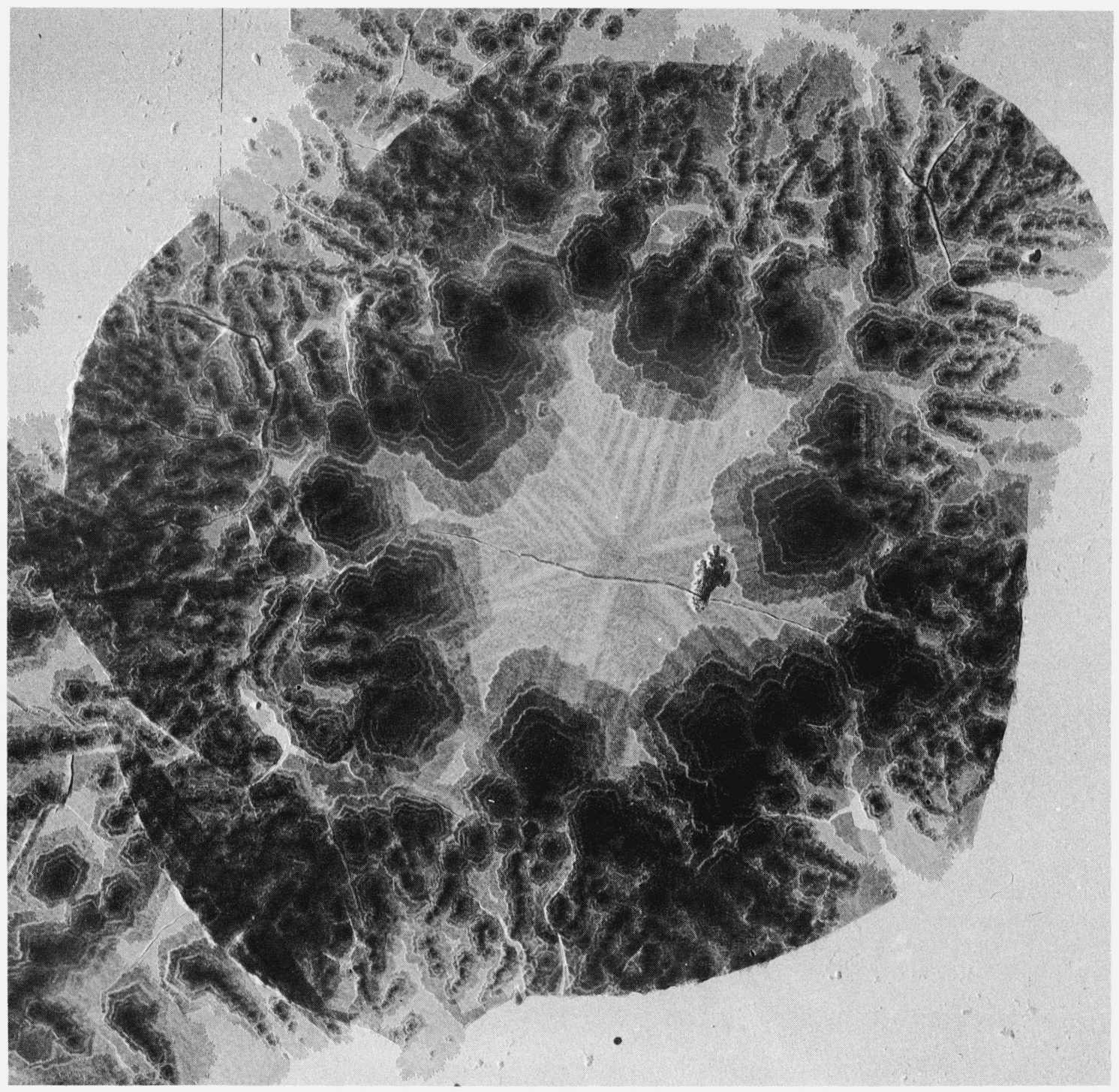

FIGURE 35. Electron micrograph showing a crystal formed in a $\mathrm{T}_{\mathrm{c}}=80^{\circ} \mathrm{C}$ preparation; see text.

$\mathrm{Pt} / \mathrm{Pd}$ shadowed, $\mathrm{X} 8500$.

120 to $114{ }^{\circ} \mathrm{C}$ or below. It is evident from a consideration and comparison of the crystals shown in figures 31 to 37 that below that temperature the lateral propagation of the crystals is characterized by the fact that as the solutions continued to cool to $T_{c}$ the initiation of new families of spiral terraces at the pe- riphery of the crystals tended to increase correspondingly as the temperature decreased. ${ }^{13}$

${ }^{13}$ There is an apparent slight reversal in this trend during the final stages of crystallization near to
liquor. 


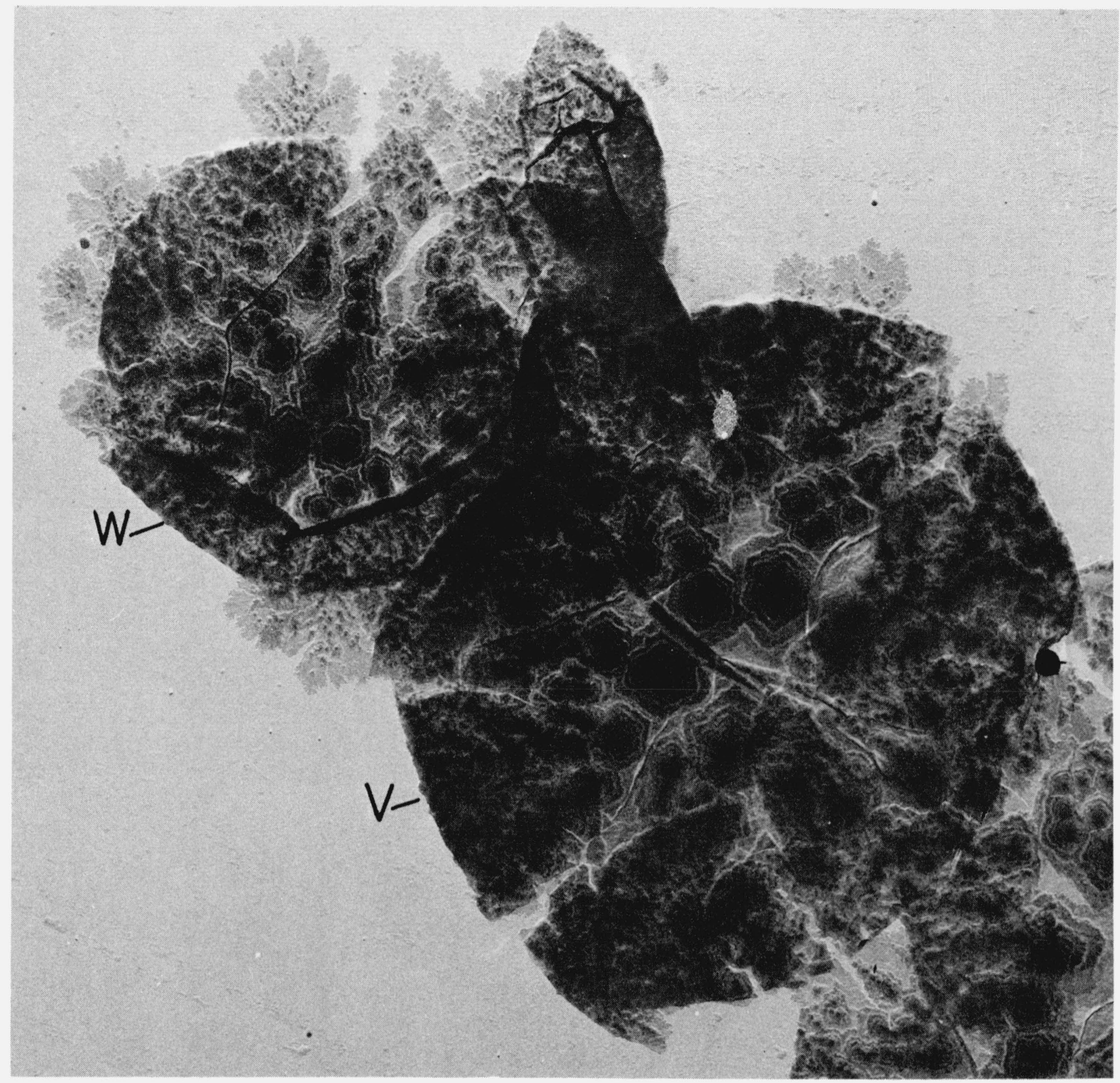

FIGURE 36. Electron micrograph showing two crystals, $(V)$ and $(W)$, formed in a $\mathrm{T}_{\mathrm{c}}=80^{\circ} \mathrm{C}$ preparation; see text.

$\mathrm{Pt} / \mathrm{Pd}$ shadowed, $\mathrm{X} 8500$.

There is an additional feature concerning the lateral propagation of the crystals below the temperature at which the onset of (SD) growth occurred during the cooling of the solutions to $T_{c}=100$ and $80{ }^{\circ} \mathrm{C}$ which should also be noted, and that is the fact that the periphery of the leading (basal) lamellar layer in the crystals as well as the periphery of the subsidiary layers (spiral terraces) in these crystals is finely serrated as may be judged from a consideration of several of the crystals shown in figures 31 to 37 and as may be seen in figure 38 in which the arrow points to a portion of the periphery of the leading (basal) chain folded layer in a crystal (formed in a $T_{c}=80^{\circ} \mathrm{C}$ preparation) as seen at a high magnification. The contrast between, the finely serrated (pronouncedly microfaceted) profile of the periphery of the leading chain folded layer in the crystals formed in these preparations on the one hand, and the more regular laterally stepped character of the basal lamella in the SD crystals formed in the $T_{c}=114{ }^{\circ} \mathrm{C}$ preparations on the 


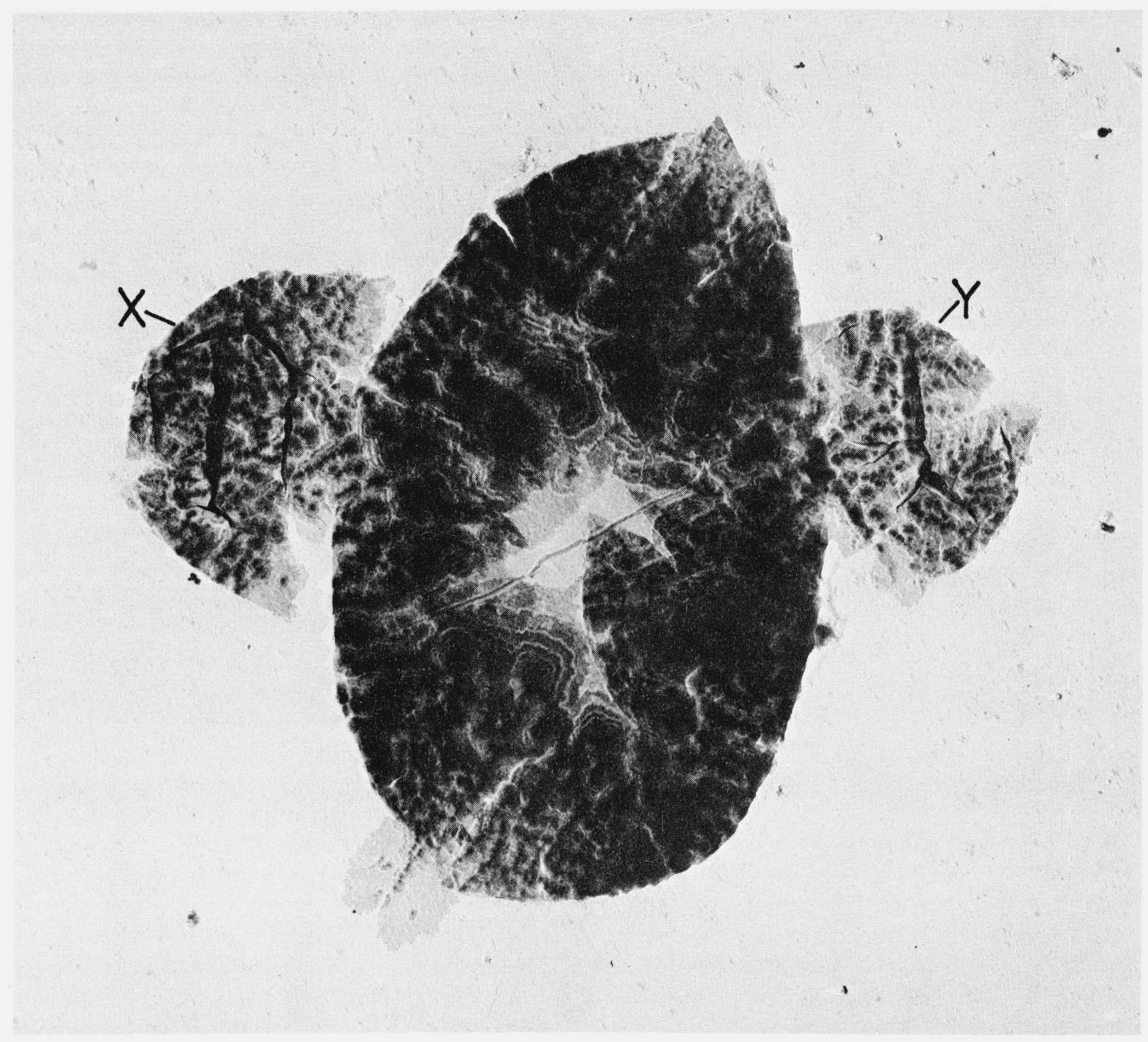

FIGURE 37. Electron micrograph showing two ( $X$ and $Y$ ) among the smaller crystals formed in a $\mathrm{T}_{\mathrm{c}}=80^{\circ} \mathrm{C}$ preparation; see text.

$\mathrm{Pt} / \mathrm{Pd}$ shadowed. X8500.

other hand, is self evident and points to the occurrence of a further transition in lateral growth habit as the temperature of the $T_{c}=100{ }^{\circ} \mathrm{C}$ and the $T_{c}=80{ }^{\circ} \mathrm{C}$ preparations decreased below the temperature at which the onset of SD growth manifested itself during the cooling of the preparations to $T_{c}$. A schematic representation of the likely nature of this transition is shown in figure $39 \mathrm{~b}$ which it is to be compared with the SD mode of growth shown in figure 39a (see also fig. la). The essential difference between the SD habit depicted in figure $39 \mathrm{a}$ and that depicted in figure $39 \mathrm{~b}$ (which will henceforth be referred to as SDD) is that whereas in the former case the initiation of new sectored outgrowths at the periphery of the basal lamella is limited to its six-leading corners (e.g., corner A in fig. 39), in the latter case new-sectored outgrowths are also initiated at subsiduary leading corners in the developing lamella such as for example corners $\mathrm{B}$ in figure $39 \mathrm{a}$ and subsequently at corners such as C in figure $39 \mathrm{~b}$, and so forth, which mode of growth involves more pronounced multisectoring than SD growth.

It is evident from the electron microscopial observations which have been described above that under the essentially similar conditions of supercooling which apparently prevailed during the crystallization of the polymer in the $T_{c}=100{ }^{\circ} \mathrm{C}$ and the $T_{c}=80{ }^{\circ} \mathrm{C}$ preparations, the history of the lateral development of individual crystals varied from crystal to crystal according to when each started to grow as the preparations cooled to $T_{c}$ due to the fact that whereas hexagonal six-sectored and/or SS growth prevailed during 


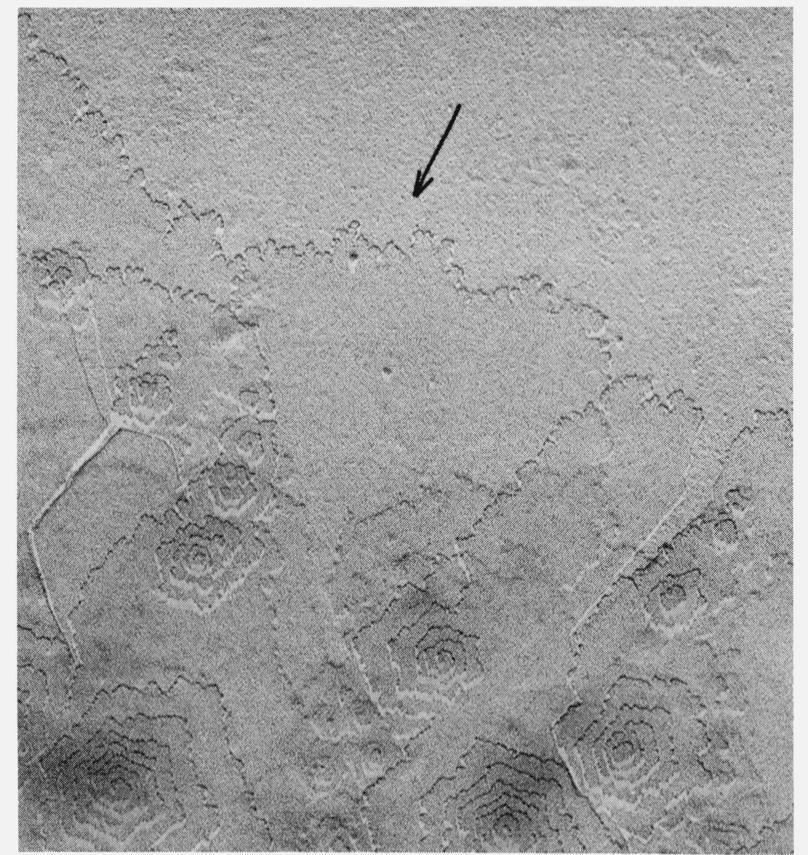

FIGURE 38. Electron micrograph ('replica') showing a portion of the outer region of a crystal formed in a $\mathrm{T}_{\mathrm{c}}=80^{\circ} \mathrm{C}$ preparation.

Arrow points to the finely serrated periphery of the crystal. $\mathrm{Pt} / \mathrm{Pd}$ shadowed. $\mathrm{X} 28100$.

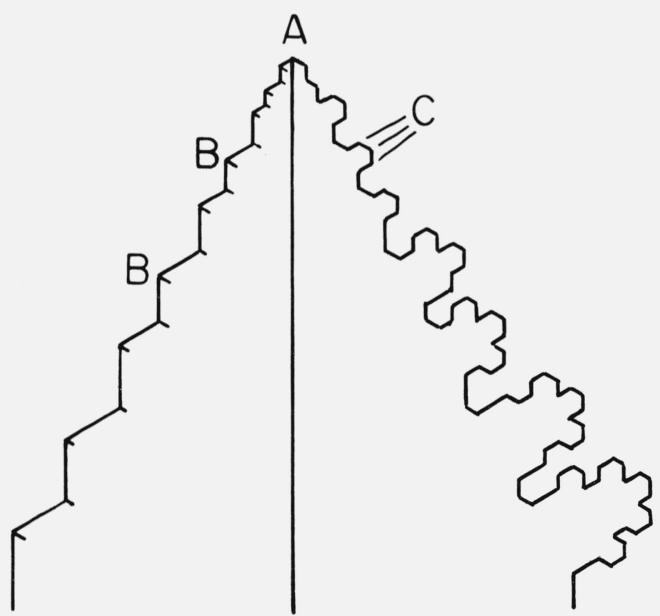

(a)

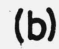

FIGURE 39. Schematic representation of (a) SD dendritic growth and $(b)$ SDD dendritic growth; see text.

the early stages of cooling, this was followed by the onset of SD growth, and subsequently SDD growth, as the temperature of the preparations decreased progressively to $T_{c}$. Accordingly, the extent of lateral propagation in the hexagonal six-sectored and SS modes was minimal (or tended towards a minimum) in the crystals which were initiated last in the preparations, in which crystals, it may also be surmised, the extent of SD growth relative to the extent of SDD growth was probably also at a minimum. It is, however, not possible to determine from the electron micrographs the stage in development at which the transition from SD to SDD growth occurred during the course of the formation of the variously sized crystals formed in these preparations. Judging from the separation between reentrant microcorners along the finely serrated periphery of the basal lamella in the crystals formed in the preparations (e.g., see fig. 38) the lateral extent of the sectored outgrowths which developed successively at the periphery of the crystals during the final stages of crystallization was approximately 0.04 $\mu \mathrm{m}$.

Summarizing the electron microscopial observations on the lateral modes of development associated with the formation of the crystals grown in the $T_{c}=100{ }^{\circ} \mathrm{C}$ and the $T_{c}=80^{\circ} \mathrm{C}$ preparations and the optical microscopial observations described earlier concerning the variations in conformation (curvature) among the crystals formed in each case, there are four main trends which emerge. First, the lateral development of the crystals, which broadly speaking involves transitions from hexagonal-six-sectored and/or SS growth, to multisectored (SD) and then (SDD) dendritic growth with decreasing temperature, is characterized by the fact that the extent of lateral growth in the hexagonal sixsectored and/or SS modes during the initial stages of development of the crystals was all the more diminished the lower the temperature at which the crystals were initiated during the cooling of the solutions to $T_{c}$. Second, the lateral development of the crystals corresponding to the multisectored SD and subsequently SDD stages of growth (which may be considered collectively for the present purpose) during the more advanced stages of the cooling of the preparation is characterized by the fact that the frequency of the formation of successive arrays of new sectored outgrowths at the periphery of the growing crystals increased with decreasing temperature, the end result being that the corresponding portions of the crystals were all the more pronouncedly multisectored (i.e., they were composed of a finer mosaic of fold domains). Judging from the separation between neighboring reentrant corners at the periphery of the leading lamella in the crystals (e.g., see fig. 38) the lateral extent of the sectored outgrowths which developed at the edges of the lamella during the final stages of growth was about $0.04 \mu \mathrm{m}$. Third, the multisectored SD and SDD stages in the growth of the leading lamella are characterized by the generation of screw dislocation-like defects at the periphery of the lamella. The incidence of these defects (which give rise to the development of spiral terraces) tended to increase the lower the growth temperature following the onset of dendritic growth. Fourth, the variations in conforma- 
tion among the crystals formed in the $T_{c}=100{ }^{\circ} \mathrm{C}$ and the $T_{c}=80{ }^{\circ} \mathrm{C}$ preparations are characterized by the fact that the crystals formed in each case were all the more pronouncedly curved the lower temperature at which they started to grow during the cooling of the solutions to $T_{c}$. There was considerable overlap between the conformations of the crystals formed in the $T_{c}=100{ }^{\circ} \mathrm{C}$ preparations and those formed in the $T_{c}=80^{\circ} \mathrm{C}$ preparations. The crystals formed in these preparations ranged from the larger species such as (N) in figure $25\left(T_{c}=100{ }^{\circ} \mathrm{C}\right.$ preparation) and $(\mathrm{Q})$ in figure $28\left(T_{c}=80^{\circ} \mathrm{C}\right.$ preparation) to small hollow bowl shaped crystals such as $(\mathrm{P})$ (diameter 5-6 $\mu \mathrm{m})$ in figure 27 in the case of $T_{c}=100{ }^{\circ} \mathrm{C}$ and such as (R) in figure 29 (diameter $4--5 \mu \mathrm{m}$ ) for $T_{c}=8{ }^{\circ} \mathrm{C}$.

Finally we turn to some additional comments concerning the conformations of the crystals formed in the $T_{c}=100{ }^{\circ} \mathrm{C}$ and $T_{c}=80{ }^{\circ} \mathrm{C}$ preparations in the light of the changes in lateral growth regime which it was pointed out above occur with decreasing growth temperature in these preparations, as well as in the light of the conformations and corresponding lateral growth characteristics exhibited by the crystals formed at all higher $T_{c}$. It has been pointed out earlier that with the qualified exception of the largest among the crystals formed in the $T_{c}=100^{\circ} \mathrm{C}$ and the $T_{c}=80^{\circ} \mathrm{C}$ preparations (such as $(\mathrm{N})$ in fig. 25 and $\mathrm{Q}$ in fig. 28) the remainder of the crystals formed in these preparations were hollow bowl shaped objects, as depicted schematically in figure 30 . Before dwelling on the overall conformation of the former crystals, the following factors pertaining to the variations in curvature among the latter six-fold symmetrical hollow bowl-shaped ones may be noted, these factors also bear on the nature of the conformation of the former larger crystals as will become evident in due course. It is evident from a comparison between, on the one hand the nearly planar six-sectored lamellar crystals formed in the $T_{c}=140{ }^{\circ} \mathrm{C}$ and the $T_{c}=120{ }^{\circ} \mathrm{C}$ preparations, and on the other hand the perceptibly dished overall character of the dendritic crystals formed in the $T_{c}=114^{\circ} \mathrm{C}$ preparations and the hollow bowl shaped dendritic crystals formed in the $T_{c}=100^{\circ} \mathrm{C}$ and $T_{c}=80^{\circ} \mathrm{C}$ preparations, that the more pronouncedly curved character of the latter dished and hollow bowl-shaped crystals as compared to the crystals formed in the $T_{c}=140{ }^{\circ} \mathrm{C}$ and $T_{c}=120{ }^{\circ} \mathrm{C}$ preparations occurs in association with the manifestation of multisectored dendritic lateral growth. It is also evident from a comparison between the dished crystals formed in the $T_{c}=114{ }^{\circ} \mathrm{C}$ preparations and the hollow bowl shape crystals formed in the $T_{c}=100{ }^{\circ} \mathrm{C}$ and $T_{c}=80^{\circ} \mathrm{C}$ preparations (as well as from the variations in curvature among the bowl-like crystals formed in the latter preparations) that the lower the temperature at which growth progressed in a dendritic manner below circa $114{ }^{\circ} \mathrm{C}$ the more pronouncedly curved were the corresponding portions of the crystals, (which trend occurred in association with a corresponding enhancement in multisectoring as well as an enhancement in the generation of screw dislocation-like defects at the periphery of the leading lamella). This trend is one of the two features which underlies the fact that the overall conformation of individual bowl-shaped crystals formed in the $T_{c}=100{ }^{\circ} \mathrm{C}$ and the $T_{c}=80^{\circ} \mathrm{C}$ preparations was all the more pronouncedly curved the lower the temperature at which the crystals started growing during the cooling of the preparations to $T_{c}$. The other feature is that the extent of lateral development in a six-sectored (and hence essentially planar) manner during the early stages of growth of these crystals was all the more diminished the lower the temperature at which they started to grow during the cooling of the solutions to $T_{c}$. Conversely in this latter connection, the higher the temperature at which a crystal started to grow the larger the extent of its lateral development in a six-sectored and nearly planar fashion prior to the onset of dendritic growth, and hence the overall curvature of the crystal tended to be less pronounced as evidenced by the larger species of crystals, such as $(\mathrm{N})$ in figure 25 and $(\mathrm{Q})$ in figure 28 , in which the incidence of pronounced curvature is confined to the outer dendritic portions of the crystals. Although the change in orientation of the surface of the larger crystals between their pole and the six leading corners of the crystals is about $90^{\circ}$ (note the "rounded hexagonal" outline of crystal Q in fig. 28), the overall conformation of these crystals is somewhat more complex than is the case of the hollow bowl-shaped crystals which started to grow at lower temperatures. This is due to the extensive separation between the six leading portions of the outer dendritic region in the larger crystals, which portions tend to have a radius of curvature comparable to that of the smallest and most pronouncedly curved crystals (e.g., (P) in fig. 27 and (R) in fig. 29) formed in the preparations. The appearance exhibited by the outer dendritic region of these larger crystals as seen in different perspectives under the optical microscope indicates that each of the six leading portions of these crystals tends to be curled in a quasi bowl-like fashion.

\section{Discussion of the Origins of the Dependence of the Curvature of POM Crystals on Crys- tallization Temperature}

The observations described in Section 3 clearly reveal a distinct trend in the effect of growth temperature on the conformation (curvature) of POM crystals. Thus, setting the axialitic among the crystals formed in the $T_{c}=140^{\circ} \mathrm{C}$ and the $T_{c}=120^{\circ} \mathrm{C}$ preparations as a species apart, it is evident that there is a progressive change in conformation from the nearly planar nature of the six-sectored monolayer and nonaxialitic multilayered crystals formed in the $T_{c}=140{ }^{\circ} \mathrm{C}$ and the $T_{c}=120{ }^{\circ} \mathrm{C}$ preparations, to the comparatively more pronouncedly curved overall conformation of the perceptibly dished dendritic crystals (radius of curvature roughly $\sim 20-30 \mu \mathrm{m}$ ) formed in the $T_{c}=114{ }^{\circ} \mathrm{C}$ preparations, to the even more pronouncedly curved overall conformations of the hollow bowl-like dendritic 
crystals formed in the $T_{c}=100{ }^{\circ} \mathrm{C}$ and the $T_{c}=80{ }^{\circ} \mathrm{C}$ preparations (radius of curvature of smallest bowls $2-3 \mu \mathrm{m})$. Noting, as pointed out in Section 3.4 that the onset of crystallization in the $T_{c}=114{ }^{\circ} \mathrm{C}$, the $T_{c}=100$ ${ }^{\circ} \mathrm{C}$ and the $T_{c}=80{ }^{\circ} \mathrm{C}$ preparations occurred before thermal equilibrium was reached at these temperatures, the results described in Section 3, looked upon collectively, may be summarized as follows: (a) A transition from nearly planar six-sectored to curved multisectored dendritic lateral growth occurred apparently in the range 120 to $114^{\circ} \mathrm{C}$, and furthermore the lower the temperature at which growth occurred thereafter the more pronouncedly multisectored were the corresponding portions of the crystals, as evidenced by the contrast between the SD profile of the periphery of the dished crystals formed in the $T_{c}=114$ ${ }^{\circ} \mathrm{C}$ preparations and the SDD profile of the periphery of the hollow bowl-shaped crystals formed in the $T_{c}=100{ }^{\circ} \mathrm{C}$ and the $T_{c}=80{ }^{\circ} \mathrm{C}$ preparations. (b) In contrast with the nearly planar character of the hexagonal six-sectored and SS crystals formed in the $T_{c}=140{ }^{\circ} \mathrm{C}$ and the $T_{c}=120{ }^{\circ} \mathrm{C}$ preparations respectively, the lower the temperature at which growth occurred (dendritically) below $T_{c}=120{ }^{\circ} \mathrm{C}$, the more pronouncedly curved were the corresponding portions of the crystals, and the greater was the tendency for screw dislocation-like defects to be generated at the periphery of the basal lamella, through the agency of which defects spiral terraces evolved.

As will become apparent, the discussion presented in what ensues on why it is that the multisectored dentritic crystals grown in the $T_{c}=114^{\circ} \mathrm{C}, 100^{\circ}$, and $80^{\circ}$ preparations were all the more pronouncedly curved the lower the crystallization temperature which prevailed during the course of their growth, in contrast with the nearly planar nature of the six-sectored monolayered and the non-axialitic multilayered crystals formed in the $T_{c}=140{ }^{\circ} \mathrm{C}$ and the $T_{c}=120{ }^{\circ} \mathrm{C}$ preparations, is rooted in considerations similar to the ones upon which our previous deliberations on the origins of curvature in P4MP crystals and its variation with crystallization temperature were based [2]. It is therefore appropriate, by way of background and for the purposes of comparison, to summarize briefly pertinent features of our previous observations on the effect of crystallization temperature on P4MP crystals grown from 0.1 percent solutions of the polymer in equivolume mixtures of xylene and amyl acetate at temperatures in the range 90 to $50{ }^{\circ} \mathrm{C}$, which crystals were all the more pronouncedly curved the lower the growth temperature. Accordingly, to indicate the extremes in the dependence of the conformations of P4MP crystals on crystallization temperature, it may be noted that the crystals varied from nearly planar ones (formed at $90{ }^{\circ} \mathrm{C}$ ) whose constituent square shaped lamellae were four-sectored, bounded laterally by $\{100\}$ faces, and only slightly buckled, to distinctly curved four-fold symmetrical hollow bowl-shaped crystals (formed in solutions cooled to $50{ }^{\circ} \mathrm{C}$ ) whose radius of curvature was $\sim 2.5 \mu \mathrm{m}$. Furthermore, in contrast with the four-sectored character of the lamellae in the essentially planar crystals formed at $90{ }^{\circ} \mathrm{C}$, the constituent lamellae in all the distinctly dished or bowl-like P4MP crystals formed in the temperature range 90 to $50{ }^{\circ} \mathrm{C}$ were multisectored, their lateral growth being characterized by the development at their periphery of successive arrays of $\{100\}$ microfaceted sectored out-growths at a frequency (per unit path length from pole to periphery) which was greater the lower the crystallization temperature. In short, in addition to the fact that the P4MP crystals were all the more pronouncedly curved the lower the growth temperature, their constituent lamellae were correspondingly all the more pronouncedly multisectored. As pointed out earlier, a similar morphological association between enhancement in curvature and increased multisectoring with decreasing growth temperature is exhibited in POM crystals grown at different temperatures. Bearing in mind this phenomenological similarity between the effect of temperature on the growth habits of POM and P4MP crystals it is also of interest to note that, as is the case for the six-sectored monolayered and non-axialitic multilayered POM crystals formed at low supercoolings $\left(T_{c}=140^{\circ} \mathrm{C}\right.$ and the $T_{c}=120^{\circ} \mathrm{C}$ preparations), the four-sectored crystals of P4MP formed at $90{ }^{\circ} \mathrm{C}$ were not perfectly planar. The slight departure from planarity in these latter crystals has been attributed to buckling under the influence of the bulkiness of the chain folds $[2,5,6]$ as is the case for the six-sectored lamellae of POM (see discussion in Section 3.2 (iii) and references [5, 6]), which attributions stem respectively from Bassett's [5] observations that $d_{\{200\}_{f}}$ differs from $d_{\{200\}_{n f}}$ by $\sim 0.1$ percent in each of the four constituent fold domains in foursectored lamellae of P4MP, and that $d_{\{10 \overline{1} 0\}_{f}}$ differs from $d_{\{10 \overline{1} 0\}}$ by $\sim 0.32$ percent in each of the six constituent fold domains in six-sectored lamellae of POM.

The similarities pointed above between the origins (the bulkiness of chain folds) of the slightly buckled character of POM (six-sectored) and P4MP (foursectored) crystals formed at low supercoolings, coupled with the phenomenological similarities between the effect of increasing supercooling on the curvature and fold domain structure in crystals of these polymers, provide the basis of the ensuing discussion of why it is that the POM crystals grown at high undercoolings were all the more pronouncedly curved the lower the growth temperature in terms of assumptions and conjectures analogous to the ones which were advanced (in the form of a working 'model', see figure 37, reference [2]) and discussed in our previous deliberations on the origins of curvature and its variation in P4MP crystals. To recapitulate briefly in this latter connection the assumptions and conjectures which were advanced and discussed in the case of P4MP were: (i) the sectored outgrowths which develop successively at the periphery of multisectored lamellae of P4MP are possessed of a slight intrinsic conicalness; (ii) this intrinsic conicalness (angle of 
elevation of $\sim 2^{\circ}$ ) in sectored outgrowths is a consequence of buckling under the influence of the bulkiness of the chain folds which leads to a difference of $\sim 0.1$ percent between $d_{\{200\}_{f}}$ and $d_{\{200\}_{n f}}$ in their constituent fold domains as is the case in four-sectored lamellae; (iii) the overall curvature of multisectored P4MP lamellae is a cumulative consequence of the intrinsic conicalness of the sectored outgrowths which develop in successive arrays at their periphery, and is governed by the frequency (per unit path length from pole to periphery) at which new sectored outgrowths are generated during the growth of the lamellae, the higher that frequency the smaller the radius of curvature of the lamellae. As suggested below a similar rationale may be advanced in the case of POM.

Given, as indicated in Section 3.2 and Section 3.3, that the six-sectored monolayered crystals and the nonaxialitic six-sectored multilayered crystals formed in the $T_{c}=140{ }^{\circ} \mathrm{C}$ and the $T_{c}=120^{\circ} \mathrm{C}$ preparations are not perfectly flat, and given as discussed in section 3.2(iii) with reference to Bassett et al. $[5,6]$, that this latter feature may be attributed to slight buckling under the influence of the bulkiness of the chain folds, i.e., that it is a consequence of both stresses which arise at the sector boundaries where the mismatch in spacing between $\{10 \overline{1} 0\}_{f}$ planes in one sector and the $\{1010\}_{n f}$ planes in adjoining sectors must be accommodated, as well as stresses at the fold surfaces, then it may be reasonably presumed that by the same token the sectored outgrowths which develop successively at the periphery of the multisectored dendritic lamellae of POM are also intrinsically slightly buckled. This latter premise would not account in itself for the fact that the multisectored dendritic crystals formed in the $T_{c}=114,100$, and $80{ }^{\circ} \mathrm{C}$ preparations were collectively more pronouncedly curved than the nearly planar six-sectored crystals formed in the $T_{c}=140{ }^{\circ} \mathrm{C}$ and the $T_{c}=120^{\circ} \mathrm{C}$ preparations, nor would it account for the fact that the dendritic crystals were all the more pronouncedly curved the lower the temperature at which growth took place below $T_{c}=120{ }^{\circ} \mathrm{C}$ during the course of their formation. This latter trend may be hypothetically accounted for on the basis of the following assumptions concerning the intrinsic conformation of sectored outgrowths, which assumptions are similar to those which were considered in our previous discussion of the formation of curved multisectored lamellar crystals of P4MP. The first assumption is that irrespective of the conditions of crystallization $d_{\{10 \overline{1} 0\}_{f}} \neq d_{\{10 \overline{1} 0\}_{n f}}$ in the constituent sectors of chain-folded lamellae of POM due to the bulkiness of of the chain folds. The second assumption is that the magnitude of the difference between $d_{\{10 \overline{1} 0\}_{f}}$ and $d_{\{10 \overline{10}\} n f}$ is the same irrespective of the conditions of crystallization, i.e., it is 0.32 percent [5] in both sixsectored lamellae as well as in the constituent sectors in the sectored outgrowths which develop successively at the periphery of the dendritic POM crystals. The third assumption is that $d_{\{10 \overline{1} 0\}_{f}}>d_{\{10 \overline{1} 0\}_{n f}}$. In short, on the basis of these assumptions it is envisaged as discussed in section 3.2(iii) that six-sectored lamellae possess a slight intrinsic conicalness, that they have an angle of inclination $(\theta)$ of a few degrees (circa $4^{\circ}$ ), that they are slightly rounded at the pole, and that the molecular stems are oriented at right angles to the surface of the crystal throughout (see fig. 8b). With these assumptions in mind, which assumptions will be the subject of further discussion in due course, we proceed to consider below the development of a multisectored dendritic lamella from an initially formed six-sectored precursor crystal.

A plan view representing a six-sectored precursor is shown schematically in figure $40 \mathrm{a}$ (efghkl) and a side view depicting the cross section of the crystal corresponding to a cut along one of its diagonals (eOh in fig. 40a) is shown in figure $40 \mathrm{~b}$ in which $\left(\mathrm{O}^{\prime} \mathrm{O}\right)$ is the normal to the surface of the lamella at its pole $(\mathrm{O})$. $\left(\mathrm{O}^{\prime} \mathrm{O}\right)$ is normal to the plane of reference $(\mathrm{mm})$ (fig. $40 \mathrm{~b})$. The normals to the surface of the lamella at (e) and $(\mathrm{h})$ are $\left(\mathrm{ee}^{\prime}\right)$ and $\left(\mathrm{hh}^{\prime}\right)$ respectively. In accordance with the visualization indicated above that the sixsectored precursor is slightly conical and rounded at the pole, it is assumed as shown in figure $40 \mathrm{~b}$, that on proceeding from its pole $(\mathrm{O})$ to its periphery following radial paths outwards along the surface, e.g., along the diagonal path $(\mathrm{Oh})$, there occurs within a small distance $(\mathrm{On})$, where $(\mathrm{On}) \ll(\mathrm{Oh})$, a gradual inclination of $\theta^{\circ}$ in the lamella relative to the plane $(\mathrm{mm})$, and that beyond (n) the inclination of the lamella remains essentially constant. In other words, it is assumed that the angle between the surface normals and hence the

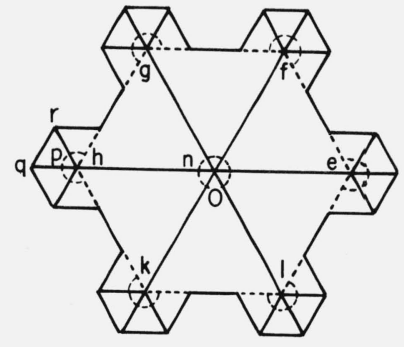

(a)

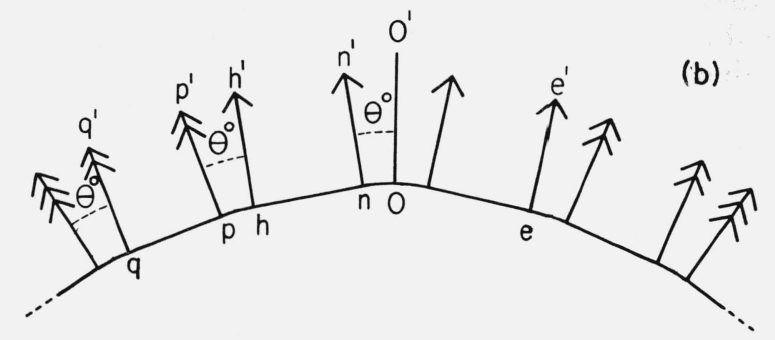

m

Figure 40(a). Plan view representing sectored outgrowths developing at the periphery of a six-sectored precursor crystal.

(b) Edge-on view of a diagonal cross-section of the developing multi-sectored dendritic crystal depicted in (a), showing conjectured incremental contribution of sectored outgrowths to the overall curvature of the lamella, see text. 
molecular stems at $(\mathrm{O})$ and $(\mathrm{n})$ is equal to the angle of inclination $\theta^{\circ}$, and that the normals (nn') and (hh') to the surface, as well as the molecular stems at (n) and (h), are parallel to one another.

With the above features attributed to a six-sectored lamella in mind, the formation is imagined of sectored outgrowths centered about the corners (efghkl) of the precursor lamella as shown in figure 40a. In essence then figure $40 \mathrm{a}$ represents an early stage in the evolution of an SD multisectored dendritic lamella of POM. As pointed out earlier it is assumed in what follows that $d_{\{10 \overline{1} 0\}_{f}}>d_{\{10 \overline{1} 0\}_{n f}}$ and that the difference between these interplanar spacings $(\sim 0.32 \%)$ is independent of the crystallization conditions and is the same in both the fold domains of the six-sectored precursor as well as in the constituent fold domains in the sectored outgrowths which develop therefrom. Accordingly, each sectored outgrowth shown in figure 40a may be considered to have a slight intrinsic "conicalness" akin to that of the central six-sectored precursor. This conicalness is not centered however about the normal to the lamella at the pole $(\mathrm{O})$ but, rather, about the surface normals at $(\mathrm{e}, \mathrm{f}, \mathrm{g}, \mathrm{h}, \mathrm{k}, \mathrm{l})$ about which the outgrowths develop. As shown crosssectionally in figure $40 \mathrm{~b}$, this conicalness is envisaged to lead to a corresponding increase in the inclination of the peripheral regions of the developing lamella relative to the plane $(\mathrm{mm})$ by an additional increment of $\theta^{\circ}$ (this increment could be as large as $2 \theta^{\circ}$ as is the case between opposite sectors in the six-sectored precursor). By analogy with the six-sectored precursor, this additional inclination is depicted in figure $40 \mathrm{~b}$ as occuring close to the center of initiation of each outgrowth e.g., between (h) and (p) where (hp) is much smaller than (hq). Thus, in this view, the total inclination of the developing multisectored lamella relative to the reference plane $(\mathrm{mm})$ on proceeding outwards from $(\mathrm{O})$ to $(\mathrm{q})$ along the surface of the crystal is envisaged to be $2 \theta^{\circ}$ as shown in figure $40 \mathrm{~b}$, i.e., an increase of $\theta^{\circ}$ in the inclination of the growing lamella relative to the plane $(\mathrm{mm})$ over that of the six-sectored precursor has resulted from the development of the inherently nonplanar sectored outgrowth centered about the precursor's periphery at $(\mathrm{h})$. The same argument applies to the other sectored outgrowths shown in figure $40 \mathrm{a}$. By the same token it may be visualized that the development of a new set of sectored outgrowths centered about the leading corners, such as (q), of the sectored outgrowths shown in figure $40 \mathrm{a}$ will lead to a further inclination $\theta^{\circ}$ of the lamella relative to the plane $(\mathrm{mm})$ and hence, cumulatively, to a total inclination of $3 \theta^{\circ}$ of the surface of the lamella (and correspondingly to a change of $3 \theta^{\circ}$ in the orientation of the molecular stems) relative to the plane $(\mathrm{mm})$ on proceeding from the pole $\mathrm{O}$ to the periphery of the newest sectored outgrowth, and so forth, the end result being that the resulting multisectored lamella will exhibit an overall curved conformation. Similar considerations apply to the case of SDD growth in which the generation of new sectored outgrowths would not be limited to the leading corners such as (q) in figure 40a but would also occur at their other corners such as (r).

Thus, as was the case in our previous discussion on the formation of variously curved crystals of P4MP [2], the thrust of the hypothetical representation of the development of curved multisectored lamellae of POM given above is that the overall curvature of the lamella is a cumulative consequence of the combined effects of two factors namely, the intrinsic conicalness of $\theta^{\circ}$ (due to the bulkiness of the chain folds) of the new sectored outgrowths which are generated successively at the periphery of the lamella during growth, and the frequency per unit path length from pole to periphery at which new sectored outgrowths are formed. Conceptually, for a constant conicalness $\theta^{\circ}$ in sectored outgrowths one would expect that the smaller the lateral dimensions which sectored outgrowths attain before new outgrowths develop therefrom in a developing multisectored lamella the larger will be the number of quasi-discrete inclinations of $\theta^{\circ}$ per unit path length from pole to periphery, consequently the average radius of curvature of the lamella will be correspondingly smaller.

The observation that the lower the temperature at which growth occurred in the basal (leading) lamella in the dendritic crystals of POM the more pronouncedly multisectored and the more pronouncedly curved were the corresponding regions of the lamella, is qualitatively consistent with the hypothetical explanation given above of the observed variations of the curvature of POM crystals formed at different supercoolings in terms of the inherently nonplanar character of sectored outgrowths and the frequency at which they are generated at the periphery of the growing crystals. However, the assumptions concerning the inherent conformation of six-sectored lamellae as well as that of sectored outgrowths upon which this hypothetical explanation is based, remain open to question. These assumptions are discussed further below.

Accepting that the premise that $d_{\{10 \overline{1} 0\}_{f}}>d_{\{10 \overline{1} 0\}_{n f}}$ in the constituent fold domains of both six-sectored lamellae as well as multisectored lamellae is correct, the possibility cannot be discounted that the disparity between $d_{\{10 \overline{1} 0\}_{f}}$ and $d_{\{10 \overline{1} 0\}_{n f}}$, which was assumed above to be independent of the conditions of crystallization, may actually differ depending on the latter conditions. In short, it may be envisaged that at low crystallization temperatures, which favor the more rapid folding of the chain molecules along the lateral facets of the sectored outgrowths which develop at the periphery of growing dendrites, the folds may tend to be less regular and hence have bulkier conformations thus giving rise to larger differences between $d_{\{10 \overline{10}\}_{f}}$ and $d_{\{10 \overline{1} 0\}_{n f}}$ and correspondingly to a more pronounced intrinsic buckling of the sectored outgrowths. In other words, the intrinsic conicalness (angle of inclination $\theta^{\circ}$ in fig. $40 \mathrm{~b}$ ) of sectored outgrowths could conceivably tend to be larger the lower the crystallization temperature. In terms of the hypothetical "model" depicted in figure 
$40 \mathrm{~b}$ this would mean that multisectored lamellae will be more pronouncedly curved the lower the crystallization temperature not only as discussed earlier, because of the larger frequency at which new sectored outgrowths are formed, and corresponding increments in inclination of $\theta^{\circ}$ occur, per unit path length from pole to periphery, but also because these increments tend to be intrinsically larger the lower the crystallization temperature. To this consideration must be added another possibility namely that even if the bulkiness of the folds and the disparity between $d_{\{10 \overline{1} 0\}_{f}}$ and $d_{\{10 \overline{0}\}_{n f}}$ are not dependent on growth temperature, the intrinsic buckling of sectored outgrowths may tend to be enhanced in some degree at lower crystallization temperatures due to the fact that the fold period and hence the thickness of sectored outgrowths become smaller the lower the crystallization temperature [6, 16].

All the considerations advanced above have been based on the premise that sectored outgrowths possess an intrinsic conicalness, which premise derives from the assumption that $d_{\{10 \overline{10}\}_{f}}>d_{\{10 \overline{1} 0\}_{n f}}$. We have described in Section 3.2 (iii) and Section 3.3 the various features - such as the streaks due to diffraction contrast, and the incidence of slight pleatswhich evidence the fact that six-sectored lamellae are not prefectly planar. We have also pointed out that it is not possible to establish with any certainty from the various "collapse features" exhibited in electron micrographs by six-sectored lamellae whether their as-grown shape, and hence as presumed earlier that of sectored outgrowths in dendritic lamellae, is intrinsically conical $\left(d_{\{1 \overline{10} \overline{0}\}_{f}}>d_{\{1 \overline{10} 0\}_{n f}}\right.$, see fig. 8) as assumed above, or whether they possess, as-grown, an intrinsic convoluted conformation such as that depicted schematically in figure $9\left(d_{\{10 \overline{1} 0\}_{f}}<d_{\{10 \overline{1} 0\} n f}\right)$. Although the matter of the exact intrinsic conformation of the six-sectored lamellae and the sectored outgrowths in the dendritic crystals remains uncertain, the overall dished and hollow bowl shaped nature of the dendritic crystals formed in the $T_{c}=114{ }^{\circ} \mathrm{C}$ preparations and in the $T_{c}=100$ and $80{ }^{\circ} \mathrm{C}$ preparations respectively tend to argue in favor of an effective slight "conicalness" in the sectored outgrowths which develop successively at their periphery.

There is a growth feature associated with the manifestation of multisectoring in the curved dendrites formed in the $T_{c}=114,100$, and $80{ }^{\circ} \mathrm{C}$ preparations which we have ignored in our discussion so far, namely the occurrence of the generation of screw dislocationlike defects about the periphery of the basal (leading) multisectored lamella in these crystals during the course of its growth. These defects, whose incidence tends to be all the more frequent the lower the growth temperature, result from a relative displacement of adjoining peripheral portions of the basal lamella in a direction perpendicular to the surface of the lamella through a distance equal to its thickness. Whether these defects which are generated preferentially at reentrant corners at the periphery of the basal lamella- which corners define the outer extremity of the contact boundary between neighboring sectored outgrowths result from a shearing of the lamella at that boundary due to localized stresses presumably associated with the bulkiness of the chain folds, or whether these defects result from built-in departures in height registry at the boundary between neighboring intrinsically buckled sectored outgrowths remains an open question. Whichever the case may be, the incidence of these screw dislocation like defects entails the occurrance of stresses resulting from their accommodation in the lattice. Whether these stresses tend to act in a manner such as to effectively enhance or diminish the intrinsic bulking of sectored outgrowths which has been proposed above, remains undetermined. It may be noted in this connection that following the occurrence of a screw dislocation-like defect at a reentrant corner the portions of the growing basal layer which were displaced relative to one another frequently heal (i.e., height registry is restored) as a consequence of the generation of a screw dislocationlike defect of opposite hand as has been previously observed [4], which feature is suggestive of a tendency for the localized distortion of the lamella associated with a single such defect to be compensated [4]. The net effect, if any, of the incidence of the screw dislocation-like defects on the conformation of the sectored outgrowths in their immediate vicinity and hence, cumulatively, the influence of these defects on the overall conformation of the curved dendrites remains unknown however, as is the possible contribution of stresses arising from the accomodation of departures in both height and lateral registry [2] between impinging (see fig. 39b) sectored outgrowths, which misregisters may be envisaged to occur (without necessarily leading to screw dislocation-like defects from which new terraces evolve) in the case of (SDD) lateral growth due to the inherently slightly buckled character of the outgrowths.

Given that the overall radius of curvature of the most pronouncedly curved hollow bowl-like crystals such as crystals $(\mathrm{R})$ in figure $29\left(T_{c}=80^{\circ} \mathrm{C}\right.$ preparation) is $\sim 2 \mu \mathrm{m}$, and assuming that the hypothetical model depicted in figure 40 in essentially correct and that the incremental inclination associated with the development of each successive sectored outgrowth at the periphery of the basal lamella is $\theta=4^{\circ}$, it may be calculated (assuming sphericity to a first approximation) that the average interval (e.g., hq in fig. 40a) between successive incremental inclinations of $\theta^{\circ}$ would be about $0.14 \mu \mathrm{m}$. In other words the lateral extent to which sectored outgrowths would be expected to develop at the periphery of the constituent basal lamella in these crystals before new sectored outgrowths evolved therefrom would be about $0.14 \mu \mathrm{m}$. This calculated estimate is greater, by a factor of three to four times the observed estimate of $\sim 0.04 \mu \mathrm{m}$ (see Sec. 3.4 (iii)) corresponding to the lateral extent of sectored outgrowths which developed during the final stages of the development of the crystals formed in the $T_{c}=100$ and $80^{\circ} \mathrm{C}$ preparations, which latter 
estimate was based on the separation between reentrant microcorners at the periphery of the basal lamella in the crystals. It may be rationalized that the experimental estimate of roughly $0.04 \mu \mathrm{m}$ corresponds to the development of the bowls at the lowest temperature at which growth occurred in the preparations and that, averaged over the entire course of the development of these most pronouncedly curved bowls, the interval between successive inclinations of $\theta^{\circ}$ may be reasonably presumed to be larger than $0.04 \mu \mathrm{m}$. The complexity of the morphology of the interior of the crystals coupled with the undetermined nature of the role of the various factors discussed in the previous four paragraphs frustrates the pursuit of this matter on a quantitative basis.

In summary, it is evident from a consideration of the conjectures upon which the hypothetical model depicted in figure 40 is based in the light of the various factors discussed in the previous four paragraphs, that these conjectures remain speculative. To recapitulate briefly, these conjectures are: (1) The primary source of the curvature of a multisectored lamella of POM is an intrinsic conicalness (angle of inclination $\theta$ circa $4^{\circ}$ ) in the sectored outgrowths which develop in successive arrays at its periphery; (ii) the intrinsic conicalness in sectored outgrowths is a consequence of buckling under the influence of the bulkiness of the chain folds which leads to a difference of about 0.32 percent between $d_{\{10 \overline{1} 0\}_{f}}$ and $d_{\{10 \overline{1} 0\}_{n f}}$ in their constituent fold domains (the buckling is envisaged to be due to stresses resulting from the accommodation of the mismatch in spacing at the fold domain boundaries between the $\{10 \overline{1} 0\}_{f}$ planes in one sector and the $\{10 \overline{1} 0\}_{n f}$ planes in adjoining ones, as well as due to stresses at the fold surfaces); (iii) the overall curvature of a multisectored lamella is a cumulative consequence of the intrinsic conicalness of the sectored outgrowths which develop in successive arrays at its periphery and is governed by the frequency per unit path length from pole to periphary at which new sectored outgrowths are generated during the growth of the crystal, the higher that frequency the smaller the radius of curvature of the crystal. ${ }^{14}$

In conclusion, the observations derived from the present study of the effect of supercooling on the curvature of POM crystals viewed in the light of our previous study on the formation of curved crystals of P4MP [2], show that there is a phenomenological similarity in the effect of supercooling on the habits

\footnotetext{
${ }^{14}$ It may be noted in connection with these conjectures which are analogous to those we previously advanced in the case of P4MP, that the study of POM reported herein, which as pointed out in Section 1 was undertaken at the outset with the specific purpose of determining whether increasing supercooling leads to the formation of POM crystals which are all the more pronouncedly curved the lower the crystallization temperature, was prompted by the evidence available at the time that (a) both six-sectored lamellae [3-6] and dendritic by the evidence available at the time that (a) both six-sectored lamellae [3-6] and dendritic
crystals [4] (see also section 1) of POM were not perfectly planar although their respective conformations remained uncertain, and that (b) the non-planar character of six-sectored lamellae was associated with the incidence of a distortion of the subcell in their constituent sectors due to the bulkiness of the chain folds $[5,6]$. These features viewed in the light of the observations and conjectures which we had previously advanced concerning the formaion of curved crystals of P4MP [2] led us to speculate that the effect of supercoling on tion of curved crystals on PAMP [2] led us to speculate that the effect of supercooling on
}

of the crystals of these two polymers. In both cases the lamellar crystals tend to be all the more pronouncedly curved the lower the crystallization temperature and the degree of multisectoring in the lamellae tends to be correspondingly all the more pronounced (i.e., the lamellae tend to be constituted of a finer mosaic of fold domains). Although the generation of screw dislocation-like defects at the periphery of the leading multisectored lamella in the crystals formed at higher supercoolings occurs in the case of both polymers, POM exhibits a pronounced propensity for the development of spiral terraces from these defects, in contrast, whereas additional chain folded terraces evolve from these defects in P4MP multisectored lamellae, there seems to be a suppression of continued "spiraling" [2]. Finally, although the observed variations in the curvature of POM and P4MP crystals with crystallization temperature lend themselves to similar hypothetical accountings, these accountings remain speculative in view of the undetermined role of numerous factors in both cases, which factors are for the most part common to both polymers.

The authors wish to express their thanks to R. K. Eby and D. H. Reneker for their encouragement and for the benefit of helpful discussions.

\section{References}

[1] The observations reported in this paper were first presented before the Division of High Polymer Physics of the American Physical Society. Khoury, F., and Barnes, J. D., Bull. Am. Phys. Soc. 12, 385 (1967), GD2.

[2] Khoury, F., and Barnes, J. D., J. Res. Nat. Bur. Stand. (U.S.), 76A (Phys. and Chem.), 225 (1972).

[3] Geil, P. H., Symons, N. K. J., and Scott, R. G., J. Appl. Phys. 30, 1516 (1959).

[4] Reneker, D. H., and Geil., P. H., J. Appl. Phys. 31, 1916 (1960).

[5] Bassett, D. C., Phil. Mag. 10, 595 (1964).

[6] Bassett, D. C., Dammont, F. R., and Salovey, R., Polymer (London) 5, 579 (1964).

[7] Geil, P. H., J. Macromol Sci. (Chem.) Al, 325 (1967).

[8] Garber, C. A., and Geil, P. H., Makromoleculare Chem. 113. 236 (1968)

[9] Carazzolo, G., J. Polymer Sci. A1, 1573 (1963).

[10] Blundell, D. J., Keller, A., and Kovacs, A. J., J. Polymer Sci. B4, 481 (1966).

[11] Bassett, D. C., and Keller, A., Phil. Mag. 7, 1553 (1962).

[12] Handbook of Chemistry and Physics, 48th Edition, The Chemical Rubber Co., Cleveland, Ohio (1967-1968).

[13] Jaffe, M., and Wunderlich, B., Kolloid-Z. u. Z. Polymere, 216-21 7, 203 (1967).

[14] Colson, J. P., and Reneker, D. H., J. Appl. Phys. 41, 4296 (1970).

[15] The manifestation of rotations between successive terraces originating from screw dislocations in chain folded polymer crystals has been discussed by Keller with particular reference to crystals of polyethylene, and of polyethylene oxide copolymers. Keller, A., Kolloid Z.u.Z. Polymere 219, 118 (1967).

[16] Korenaga, T., Hamada, and F., Nakajima, A., Polymer Journal (Japan) 3, 21 (1972).

(Paper 78A2-805) 